Der Medizinischen Fakultät der Georg-August-Universität eingereicht von Prof. Dr. med. habil. em. L. Adler

\title{
Kontentanalytische Studie zu Amokfahrten im Spektrum schwerer Verkehrsunfälle mit Personenschäden aus deutschen Presseberichten
}

\author{
INAUGURAL-DISSERTATION \\ zur Erlangung des Doktorgrades \\ der Medizinischen Fakultät der \\ Georg-August-Universität Göttingen
}

vorgelegt von

Barbara Werneburg

aus

Treffurt

Göttingen 2020 
Dekan:

Referent/in

Ko-Referent/in

Datum der mündlichen Prüfung:

30.03 .2021
Prof. Dr. med. W. Brück

Prof. Dr. med. habil. em. L. Adler

Prof. Dr. med. Thomas Meyer 
Hiermit erkläre ich, meine Dissertationsarbeit mit dem Titel „Kontentanalytische Studie zu Amokfahrten im Spektrum schwerer Verkehrsunfälle mit Personenschäden aus deutschen Presseberichten“ eigenständig angefertigt und keine anderen als die von mir angegebenen Quellen und Hilfsmittel verwendet zu haben.

Göttingen, den 01.10 .2020 


\section{Inhaltsverzeichnis}

Tabellenverzeichnis .......................................................................................................................... III

Abbildungsverzeichnis...........................................................................................................

1. Einleitung........................................................................................................................ 1

1.1 Aggressivität im Straßenverkehr..................................................................... 4

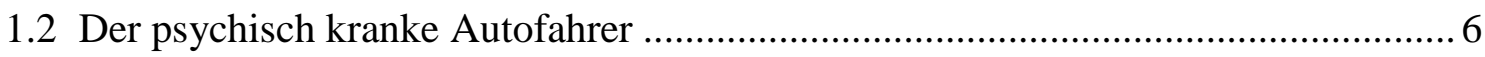

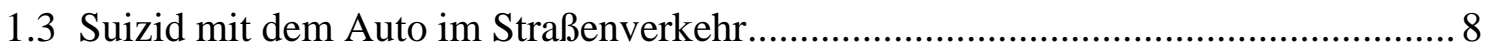

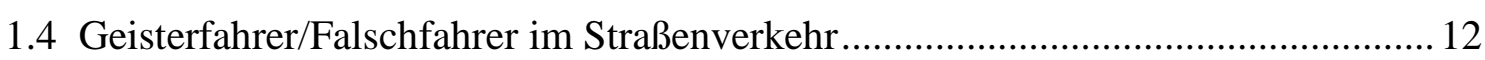

1.5 Die Amokfahrt als Sonderform von Amok im Straßenverkehr ................................ 17

1.6 Fragestellungen und Ausgangshypothese ……………………………………...... 21

2. Material und Methoden ...............................................................................................................21

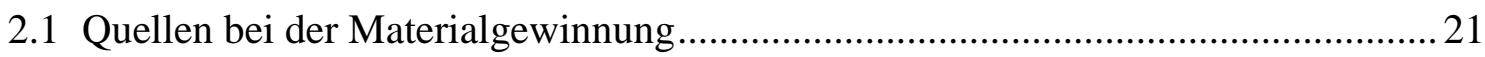

2.2 Datenlage zu Berichten über Amokfahrten in lokalen Zeitungen des Freistaates

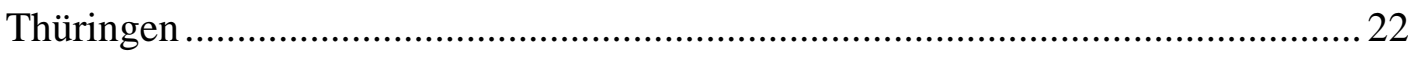

2.3 Datenlage zu Berichten über schwere Verkehrsunfälle durch Amokfahrer in der überregionalen Presse ……………………………………............................... 23

2.4 Berichte über Amokfahrer in der deutschen Presse aus dem europäischen Ausland

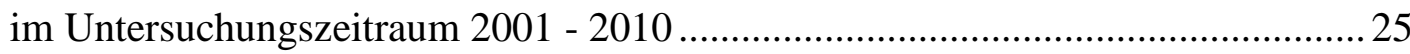

2.5 Berichte über Amokfahrer in der deutschen Presse aus dem weltweiten Geschehen

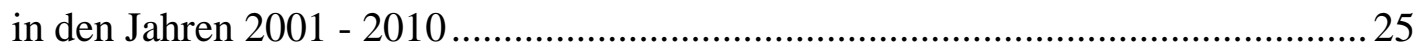

2.6 Datenlage zur Gesamtzahl von Amokfahrten in allen drei untersuchten Dekaden

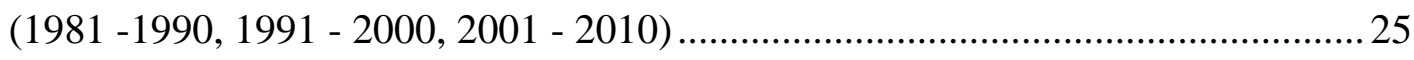

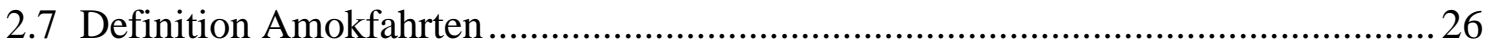

2.8 Einschluss- und Ausschlusskriterien bei Amokfahrten ............................................2 27

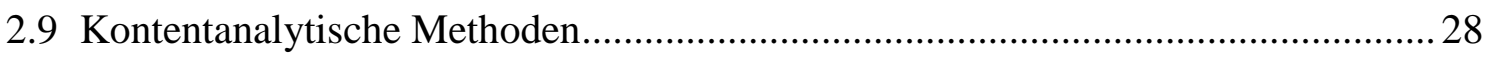

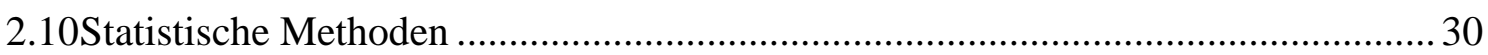

3. Ergebnisse .........................................................................................................................32

3.1 Amokfahrten im Spektrum anderer schwerer Verkehrsdelikte im Freistaat

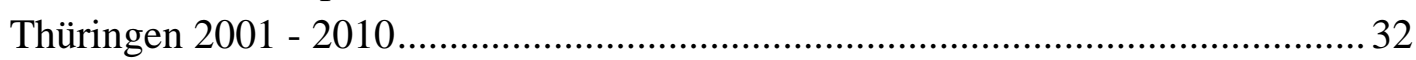

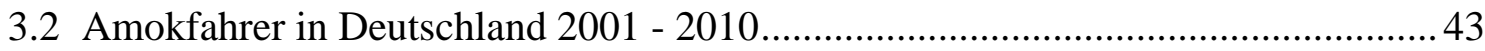

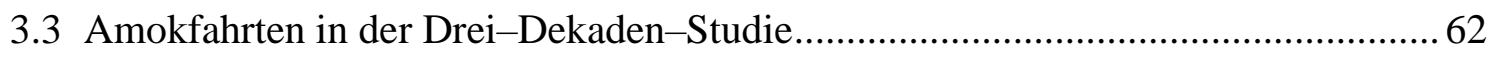

3.4 Berichte über Amokfahrten im europäischen Ausland im Zeitraum 2001 - 2010... 64

3.5 Berichte in der deutschen Presse über außereuropäische Amokfahrten im

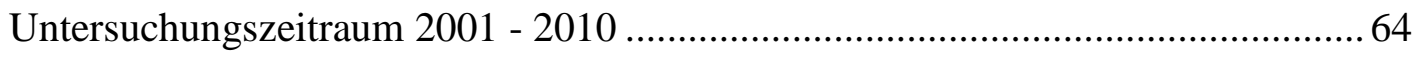

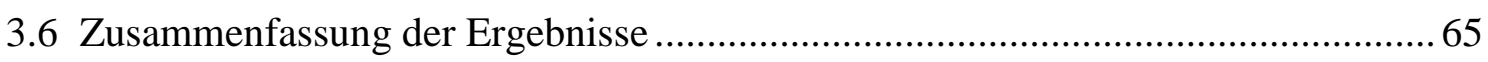

4. Diskussion ......................................................................................................................................67 
4.1 Soziodemographische Charakterisierung der Amokfahrer 67

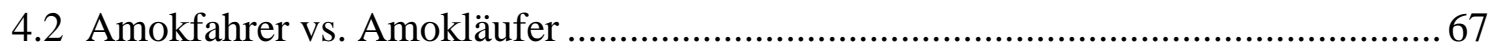

4.3 Amokfahrer in der 3-Dekaden-Studie in Deutschland...........................................69

4.4 Abgrenzung von Amokfahrten gegenüber anderen Formen gefährlichen

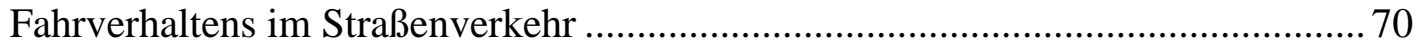

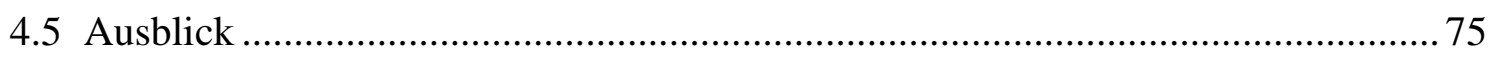

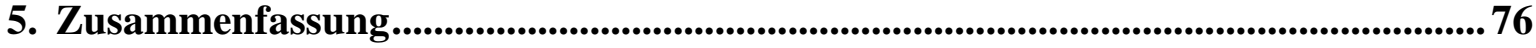

6. Anhang .................................................................................................................................................. 79

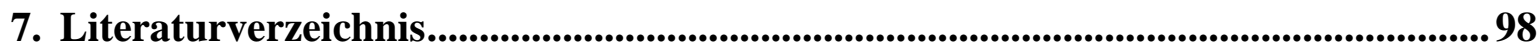




\section{Tabellenverzeichnis}

Tabelle 1: Vergleich Amokläufer und Amokfahrer ........................................................... 19

Tabelle 2: Schwere Verkehrsunfälle mit Personenschäden in Thüringen 2001-2010 ...........32

Tabelle 3: Erfasste Verkehrsunfälle mit Personenschäden in Deutschland...........................43

Tabelle 4: Altersverteilung aller Amokfahrer in Deutschland...............................................4 44

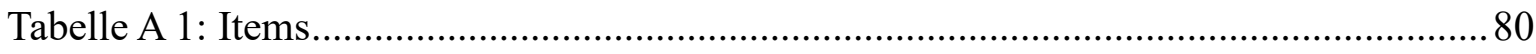

Tabelle A 2: Auflistung der ausgewerteten Presseartikel ................................................... 88 


\section{Abbildungsverzeichnis}

Abbildung 1: Darstellung der Häufigkeiten der Amokfahrten in Deutschland.

Abbildung 2: Altersverteilung der Amokfahrer

Abbildung 3: Familienstand der Amokfahrer

Abbildung 4: Berufsausbildung der Amokfahrer

Abbildung 5: Beschäftigungsverhältnis der Amokfahrer. 46

Abbildung 6: Persönlichkeitsauffälligkeiten ....

Abbildung 7: Psychiatrische und/oder Suchterkrankung vor der Tat .48

Abbildung 8: Tatvorfeld 48

Abbildung 9: Geschlechtsverteilung....

Abbildung 10: Herkunft der Amokfahrer 49

Abbildung 11: Tatmotive 51

Abbildung 12: Zeit - Tatmotiv-Beziehung.... 51

Abbildung 13: Konfliktbezogene Täter - Opfer-Beziehung 52

Abbildung 14: Persönliche Täter - Opfer Beziehung. 52

Abbildung 15: Anzahl der Fahrten pro Uhrzeit 53

Abbildung 16: Tatdauer. 54

Abbildung 17: Tatortwechsel 54

Abbildung 18: Todesopferzahl ... 55 
Abbildung 19: Verletztenzahl pro Fall

Abbildung 20: Opfergeschlecht 56

Abbildung 21: Opferalter 57

Abbildung 22: Sachschaden. 57

Abbildung 23: Fluchtverlauf. 58

Abbildung 24: Ende Amokfahrt 58

Abbildung 25: Art der Diagnosestellung... 59

Abbildung 26: Diagnosestellung nach dem Deutschen Schichtenmodell. ... 60

Abbildung 27: Akute Intoxikation 61

Abbildung 28: Angaben des Täters 62

Abbildung 29: Anzahl der Amokfahrten deutschlandweit in den jeweiligen Dekaden 63 


\section{Einleitung}

Allein das Wort Amokfahrt auf der Titelseite einer Zeitung oder als Überschrift eines Presseartikels löst beim Leser fast automatisch eine Vorstellung von katastrophalen Verkehrsunfällen mit Toten, Schwerverletzten und hohen Sachschäden aus. Und da in den letzten Jahren subjektiv der Eindruck entstand, dass in den Medien häufiger von dort so genannten Amokfahrten berichtet wurde als in der Vergangenheit, legte dies die Vermutung nahe, dass dadurch die Gefahr für Verkehrsteilnehmer, Opfer einer Amokfahrt zu werden, größer geworden ist. Allerdings muss man sich an dieser Stelle auch fragen, ob der öffentliche Straßenverkehr jemals frei von Gefahren war - wohl kaum. Öffentlichen Straßenverkehr mit motorisierten Fahrzeugen gibt es weltweit seit mehr als 100 Jahren. Anfangs waren Automobile längst nicht so schnell wie heute und waren auf den Straßen weitaus seltener anzutreffen. Die Autos von damals waren technisch noch unausgereift, störanfällig und unfallträchtig. Heute stellt der motorisierte Straßenverkehr die Hauptform des öffentlichen und Individualverkehrs dar.

Die heutigen Kraftfahrzeuge sind technisch weitestgehend ausgereift und werden regelmäBig auf ihre Verkehrssicherheit überprüft. Trotz oft hoher Verkehrsdichte in den Ballungsgebieten fließt der Verkehr auch dort reibungslos, wenn sich alle Verkehrsteilnehmer an die allgemein gültigen Verkehrsregeln halten. $\mathrm{Zu}$ diesen Regeln zählt unter anderem das Rechtsfahrgebot (analog dazu das Linksfahrgebot in Großbritannien und den Commonwealth-Ländern). Wird es nicht eingehalten (z. B. durch einen Geisterfahrer), kann es schlimmstenfalls zu einem Frontalzusammenstoß mit katastrophalen Folgen für alle beteiligten Verkehrsteilnehmer kommen. Setzt sich ein Autofahrer morgens in sein Fahrzeug, um zur Arbeit zu fahren, denkt er normalerweise nicht an die Gefahren, denen er sich durch seine Teilnahme am Straßenverkehr aussetzt. Diese Verdrängungsleistung ist sinnvoll und notwendig, denn für die meisten berufstätigen Menschen, insbesondere im ländlichen Raum, gibt es keine praktikable Alternative zur täglichen Fahrt zur Arbeit mit dem Auto.

Wenn Menschen, die täglich mit dem Auto unterwegs sind, in der Zeitung Berichte über schwere Verkehrsunfälle lesen, sind sie dadurch zumeist nicht übermäßig beunruhigt, solange nicht eine ihnen bekannte Person in den Unfall involviert und der Unfallhergang nachvollziehbar ist. Ist ein Unfall jedoch Folge einer vom Unfallverlauf her irrational und nicht nachvollziehbar erscheinenden Amokfahrt, so funktionieren nun die gewöhnlichen 
Verdrängungsmechanismen nicht mehr und die bis dahin nicht wahrgenommenen (weil verdrängten) Verkehrsgefahren gelangen nun schlagartig wieder ins Bewusstsein zurück. Die Folge davon ist eine erhebliche Beunruhigung und Verunsicherung in der Bevölkerung darüber, wie groß die Gefahr tatsächlich ist, die Amokfahrten für den öffentlichen Straßenverkehr und den einzelnen Verkehrsteilnehmer darstellen und wie häufig mit dem Auftreten von Amokfahrten im Straßenverkehr zu rechnen ist. Spektakuläre Presseberichte über Amokfahrt genannte Verkehrsereignisse mit Toten, Verletzten und hohen Sachschäden können dem Leser den Eindruck vermitteln, als ob er im Straßenverkehr zunehmend häufiger auf gefährliche Verkehrsteilnehmer wie Amokfahrer treffen kann, die sein Leben in Gefahr bringen können.

Durch Presseberichte über teilweise ganze Serien so genannter Amokfahrten im öffentlichen Straßenverkehr, die neben den Amokläufen in der Drei-Dekaden-Amokstudie von Adler et al. (1993; 1994; 2001; 2010a) als Amoktat mit einer irregulären Waffe wiederholt in Erscheinung traten, entstand ein wissenschaftliches Interesse mit Fragestellungen wie: Ist in den letzten Jahren die Zahl der Amokfahrten auf deutschen Straßen tatsächlich angestiegen? Was wissen wir über die Persönlichkeit und Psychopathologie des Amokfahrers gleicht sie dem Amokläufer oder gibt es Unterschiede zwischen Amokläufer und Amokfahrer? Aus diesen Fragen wurden die Untersuchungsschwerpunkte dieser Arbeit abgeleitet, die sich damit beschäftigt, unter vornehmlich psychiatrischen Gesichtspunkten verschiedene Formen gefährlichen Verhaltens im Straßenverkehr mit dem Schwerpunkt Amokfahrten zu untersuchen und gegeneinander und dabei insbesondere gegen die echte Amokfahrt abzugrenzen, mit Hilfe der gewonnenen Untersuchungsergebnisse Aussagen über die tatsächliche Häufigkeit von Amokfahrten im öffentlichen Straßenverkehr zu treffen sowie darüber, welchen Einfluss Persönlichkeitsakzentuierungen, umschriebene psychische Erkrankungen und weitere sozio-psycho-biologische Faktoren auf das gefährliche Fahrverhalten der verschiedenen Fahrertypen haben.

Bevor mit den eigenen Untersuchungen begonnen wurde, erfolgte die Auseinandersetzung mit dem der wissenschaftlichen Literatur, in der sich Autoren aus verkehrstechnischer, verkehrspsychologischer, kriminalistischer und psychiatrischer Sicht mit besonders unfallträchtigen Verkehrsteilnehmern und weiteren Ursachen schwerer Verkehrsunfälle mit Personenschäden beschäftigt hatten. Bereits bei der Literaturrecherche zeigte sich, dass es außer dem (echten) Amokfahrer noch weitere gefährliche und besonders unfallträchtige Autofahrer gibt, die in vier eigenständige Gruppen (aggressiver Autofahrer, psychisch 
kranker Autofahrer, Suizidfahrer, Geisterfahrer) geteilt wurden. Hinsichtlich ihrer Gemeinsamkeiten und Unterschiede wurden sie dem Amokfahrer - dem eigentlichen Gegenstand dieser Untersuchung - gegenübergestellt und auch miteinander verglichen. Die Ergebnisse der Literaturrecherche wurden danach mit den eigenen Untersuchungsergebnissen in Beziehung gesetzt und diskutiert. Im Rahmen der eigenen Untersuchungen erfolgte eine Totalerhebung schwerer irrationaler Verkehrsunfälle mit Personenschäden, die sich innerhalb eines definierten 10-Jahres-Zeitraums auf dem Gebiet des Freistaates Thüringen ereignet hatten und über die in der lokalen Presse berichtet worden war. Ursprünglich war geplant, die in dieser Totalerhebung verifizierten Fälle echter Amokfahrten mit Hilfe einer kontentanalytischen Untersuchung auszuwerten, was aber aufgrund der sehr kleinen Thüringer Fallzahlen hinsichtlich einer statistischen Auswertung nicht durchführbar war. Um sie schließlich doch auswerten zu können, wurde beschlossen, die Thüringer Amokfahrten in die Gesamtgruppe der Amokfahrten, die sich im selben Untersuchungszeitraum auf dem Gebiet der Bundesrepublik Deutschland ereignet hatten, eingehen zu lassen. Zusätzlich wurden Presseberichte über Amokfahrten, die sich im europäischen Ausland und in anderen Teilen der Welt ereignet hatten (soweit im Untersuchungszeitraum in der deutschen Presse über sie berichtet wurde), in die Untersuchung aufgenommen. Da diese ebenfalls aufgrund ihrer geringen Fallzahlen statistisch nicht auswertbar waren, gingen sie in die Falltypisierung ein.

Ziel dieser Arbeit war, das Auftreten von Amokfahrten - und im Vergleich dazu anderes zumindest äußerlich homizidal-suizidal erscheinendes gefährliches Fahrverhalten - im Straßenverkehr Hypothesen generierend zu untersuchen, Aussagen treffen zu können über die tatsächliche Häufigkeit ihres Auftretens und - was das Auftreten echter Amokfahrten betrifft - einen Beitrag zur Amokforschung im Rahmen der schon genannten AmokLangzeitstudie zu leisten. 


\subsection{Aggressivität im Straßenverkehr}

Sowohl Whitlock (1971) wie auch Michalowski (1975) und Macmillan (1975) konnten in ihren Untersuchungen zeigen, dass es sich bei Autofahrern mit einem aggressiven unfallträchtigen Fahrstil überdurchschnittlich häufig um junge Männer handelt. Während Whitlock (1971) bei diesen jungen männlichen Autofahrern manifeste Anzeichen eine Persönlichkeitsstörung vom aggressiven und/oder antisozialen Typ feststellte, beschrieb Michalowski (1975), der in seiner Studie 119 junge männliche Autofahrer untersuchte, die mit ihrer aggressiven Fahrweise schwere Verkehrsunfälle mit tödlichem Ausgang verursacht hatten, dass etwa die Hälfte dieser jungen Autofahrer zum Unfallzeitpunkt unter erheblichem Alkoholeinfluss gestanden hatte. Macmillan (1975) hingegen erklärte die vermehrte Unfallträchtigkeit junger männlicher Autofahrer durch eine Kombination von aggressivem Fahrstil, geringer Fahrerfahrung und Konkurrenzverhalten zu anderen Autofahrern. Mayer (1987), der in einer deutlich kleineren Studie 30 junge männliche Autofahrer, die überdurchschnittlich häufig Verkehrsunfälle verursacht hatten, mit einer gleich großen Gruppe unfallfreier Fahrer verglich, konnte zeigen, dass sich in der UnfallfahrerGruppe deutlich mehr Personen mit einer antisozialen Persönlichkeit, mit einer Impulskontrollstörung sowie mit Alkohol- und/oder Drogenmissbrauch befanden. Einen ähnlichen Zusammenhang zwischen erhöhter Unfallhäufigkeit von Autofahrern und bestimmten Persönlichkeitseigenschaften (traits) fanden auch Grey et al. (1989): Sie konnten zeigen, dass bei Autofahrern, die wiederholt schwere Unfälle verursacht hatten, überdurchschnittlich häufig Persönlichkeitseigenschaften wie übermäßige Aggressivität, Feindseligkeit, Rücksichtslosigkeit, Impulsivität und erhöhte Risikobereitschaft vorhanden waren. Greiner (1995) wies aus kriminologischer Sicht auf den Zusammenhang hin, dass Personen, die auch schon zuvor durch aggressives Verhalten auch strafrechtlich in Erscheinung getreten waren, im Straßenverkehr sehr häufig als rücksichtslose Autofahrer auffallen. Im Ergebnis ihrer umfangreichen Studie über das Fahrverhalten von 520 schwedischen Autofahrern konnten Åberg und Rimmö (1998) ähnlich wie schon zuvor Macmillan (1975) und Michalowski (1975) zeigen, dass junge männliche Autofahrer aufgrund ihres oft riskanten Fahrverhaltens deutlich mehr Verkehrsunfälle verursachen als alle übrigen Autofahrer der Gesamtgruppe. Auch Adelt et al. (1999) kamen in ihrer Untersuchung zu dem Ergebnis, dass mehr als $80 \%$ aller 18 - 25 jährigen männlichen Autofahrer aufgrund ihres Fahrverhaltens ein deutlich höheres Risiko aufweisen, schwere Verkehrsunfälle zu verursachen. Aus Sicht dieser Autoren erklärt sich das erhöhte Unfallrisiko der jungen männlichen Auto- 
fahrer mit dem alterstypischen Ausleben von Risiko- und Abenteuerlust beim Autofahren sowie zusätzlich noch aus der gefährlichen Kombination von jugendlicher, sportlichrasanter Fahrweise und noch geringer Fahrpraxis. Das Phänomen des jungen männlichen Autofahrers als häufigem Verursacher schwerer Verkehrsunfälle mit Personenschäden nimmt in der Literatur einen breiten Raum ein: Wie bereits Whitlock (1971) in einer frühen Arbeit, so kamen auch Fong et al. (2001) in ihrer Arbeit zu dem Ergebnis, dass die größte Gruppe der Unfallverursacher im Straßenverkehr jünger als 30 Jahre alt ist und zumeist geringere Ausbildungsabschüsse aufweist, als der durchschnittliche Autofahrer. Die Ergebnisse ihrer testpsychologischen Untersuchung jüngerer Unfallverursacher mit dem Aggression-Questionaire-Test machten deutlich, dass die jungen Männer der Unfallverursacher-Gruppe signifikant höhere Aggressionswerte aufwiesen, als die Fahrer der Vergleichsgruppe. Auch Maag und Krüger (2003) als Verkehrswissenschaftler wiesen in ihrer Untersuchung nach, dass Aggression und aggressives Autofahren im öffentlichen Straßenverkehr vor allem ein Problem von jungen männlichen Autofahrern ist. Sie bezeichneten das Fahrverhalten dieser Autofahrer-Gruppe als rücksichtslos-egoistisch und instrumentellaggressiv. Eine der umfangreichsten Studien über aggressives Verhalten im Straßenverkehr führten Nabi et al. (2006) durch: Sie untersuchten über einen Zeitraum von 8 Jahren 11.000 französische Autofahrer, die die so genannte GAZEL-Kohorte bildeten. Auch diese Autoren konnten mittels testpsychologischer Untersuchungen einen Zusammenhang zwischen den Persönlichkeitseigenschaften eines Autofahrers (hier: das individuelle Ausmaß an Aggressivität und Feindseligkeit) und der Häufigkeit, mit der er schwere Unfälle im Straßenverkehr verursachte, nachweisen. Nabi et al. konnten zeigen, dass jüngere männliche, nur gering ausgebildete und überdurchschnittlich aggressiv und feindselig auftretende Autofahrer deutlich mehr Verkehrsunfälle verursachen als die durchschnittliche Autofahrerpopulation. Weiterhin wiesen die Autoren nach, dass auch aggressiv fahrende männliche Autofahrer jenseits des Jugendalters (die sogenannten „Raser“) sowie Auto fahrende alkohol- und/oder Drogen intoxikierte Personen beiderlei Geschlechts ebenfalls überdurchschnittlich viele Verkehrsunfälle verursachen. Laux und Brunnauer (2010) prägten in der deutschsprachigen Verkehrsliteratur für diese besonders unfallträchtigen Autofahrer den Begriff der „Unfäller-Persönlichkeit“ - angelehnt an den seinerzeit von Tabachnick (1973) geprägten Begriff der accident prone personality. Als „Unfäller-Persönlichkeit" definierten Laux und Brunnauer (2010) die Persönlichkeit eines Autofahrers, der durch sein soziopathisch-rücksichtsloses (in einer selteneren Ausprägung auch depressiv-destruktives) Fahrverhalten für den öffentlichen Straßenverkehr eine besondere Gefahr darstellt. Zu- 
sammenfassend lässt sich feststellen, dass die Häufigkeit, schwere Unfälle zu verursachen, in der Gesamtpopulation der Autofahrer im öffentlichen Straßenverkehr ungleich verteilt ist: Die meisten Autoren (Adelt et al. (1999), Fong et al. (2001), Laux und Brunnauer (2010), Maag und Krüger (2003), MacMillan (1975), Mayer (1987), Michalowski (1975), Whitlock (1971) bezeichnen als Hochrisiko-Gruppe für das Verursachen schwerer Verkehrsunfälle mit Personenschäden junge männliche Autofahrer sowie Autofahrer mit Persönlichkeitseigenschaften (traits) wie: Vermehrte Aggressivität, Impulsivität, Rücksichtslosigkeit und erhöhte Risikobereitschaft. Deren Risiko, im öffentlichen Straßenverkehr einen schweren Unfall zu verursachen, ist überdurchschnittlich hoch und steigt bei zusätzlichem Fahren unter Alkohol- und/oder Drogeneinfluss nochmals weiter an.

\subsection{Der psychisch kranke Autofahrer}

In seiner Übersichtsarbeit beschäftigte sich Gordon (2004) mit der Frage der Unfallhäufigkeit von psychiatrischen Patienten, die gleichzeitig als Autofahrer im öffentlichen Straßenverkehr unterwegs sind. Er ging dabei auch auf eine frühe Arbeit von Waller ein (Waller 1965, zitiert in Gordon 2004), der in seinen Untersuchungen zu dem Ergebnis kam, dass Autofahrer, die an einer schwerwiegenden akuten psychischen Erkrankung leiden, etwa doppelt so viele Unfälle verursachen wie die psychisch gesunden Probanden der Vergleichsgruppe. Gordon (2004) setzte in seiner Untersuchung die Arbeit von Waller (1965) in Beziehung zu den Untersuchungen von Harris (Harris 2000, zitiert in Gordon 2004), der außerdem noch zeigen konnte, dass sich das erhöhte Unfallrisiko von Autofahrern mit einer psychischen Erkrankung nach erfolgreicher medikamentöser Behandlung wieder normalisiert und danach vom Unfallrisiko der Personen der Vergleichsgruppe nicht mehr zu unterscheiden ist. Kastrup et al. (1978) gingen in ihrer Untersuchung der Frage nach, ob nicht psychische Erkrankung an sich, sondern die bei den jeweiligen psychisch kranken Autofahrern gestellten Diagnosen hinsichtlich der Unfallhäufigkeit unterschiedlich zu bewerten sind. In einer 3-Jahres-Studie werteten die Untersucher die Daten von 2020 Personen aus, die sowohl im Dänischen Verkehrsunfall-Zentralregister als auch im Psychiatriepatienten-Zentralregister von Dänemark erfasst worden waren. Sie kamen zu dem Ergebnis, dass bei den registrierten Psychiatriepatienten, die gleichzeitig im Dänischen Verkehrsunfall-Zentralregister als Unfallverursacher erfasst waren, als häufigste Diagnose eine schwere Persönlichkeitsstörung bestand (22,2 \%), gefolgt von den Diagnosen Alko- 
hol- und Drogen-Abusus (15,8 \%), schwere neurotische Störung (14,7 \%) sowie akute Belastungsreaktion (13,2\%). Anders als erwartet gab es deutlich weniger Unfallverursacher, bei denen die Diagnosen manisch-depressive Erkrankung (7,7\%), Schizophrenie (1,6 \%) oder paranoide Psychose $(1,1 \%)$ vorlagen. Ein weiteres überraschendes Ergebnis der Untersuchung war, dass $97 \%$ der untersuchten dänischen Psychiatrie-Patienten nur ein einziges Mal in ihrem Leben krankheitsbedingt zu Unfallverursachern wurden. Nur $3 \%$ der Personen dieser Gruppe verursachten mehrfach schwere Verkehrsunfälle. Bei diesen handelte es sich ausschließlich um Personen mit der Diagnose schwere Persönlichkeitsstörung. Shinar (1978) stellte in seiner Untersuchung über Autofahrer mit der Diagnose schwere Persönlichkeitsstörung ebenfalls fest, dass das Unfallrisiko dieser Personengruppe deutlich höher ist als das der Autofahrer-Normalpopulation. Des Weiteren konnte der Autor zeigen, dass das bereits erhöhte Unfallrisiko von Autofahrern mit schweren Persönlichkeitsstörungen noch weiter ansteigt, wenn sie gleichzeitig alkoholisiert im Straßenverkehr unterwegs sind. Auch Fong et al. (2001) konnten einen Zusammenhang zwischen abnormen Persönlichkeitsmerkmalen, Substanzmissbrauch und besonders unfallträchtigem Fahrverhalten nachweisen. Nabi et al. (2006) kamen in ihrer Studie ebenso zu dem Ergebnis, dass von Autofahrern mit einer aggressiv-feindseligen Persönlichkeit bei gleichzeitig bestehendem Alkoholabusus und Fahren unter Alkohol eine nicht unerhebliche Gefahr ausgeht, überdurchschnittlich viele schwere Verkehrsunfälle mit Personenschäden zu verursachen. Zusammenfassend bedeutet das, dass die Häufigkeit der Verursachung schwerer Verkehrsunfälle von Autofahrern mit einer psychischen Erkrankung oder Störung differenziert betrachtet werden muss: Leiden Autofahrer an einer akuten noch unbehandelten psychischen Erkrankung, so ist ihr Risiko, im Straßenverkehr einen schweren Verkehrsunfall mit Personenschäden zu verursachen, doppelt so hoch wie bei gesunden Vergleichsprobanden (Gordon 2004). Das deutlich erhöhte Unfallrisiko dieser Autofahrergruppe normalisiert sich allerdings wieder nach ausreichender Behandlung der Grunderkrankung (ebenda). Nur eine sehr kleine Gruppe psychisch auffälliger Autofahrer hat ein dauerhaft erhöhtes Unfallrisiko. Es handelt sich dabei um Personen mit einer schweren Persönlichkeitsstörung (insbesondere vom aggressiven und impulsiven Typ). Das Risiko, schwere Unfälle mit Personenschäden zu verursachen, steigt bei dieser Personengruppe bei zusätzlichem chronischem Substanzmissbrauch und Fahren unter Alkohol und/oder Drogen noch weiter an (Fong et al. 2001, Kastrup et al.1978, Shinar 1978). 


\subsection{Suizid mit dem Auto im Straßenverkehr}

Bereits Ford und Moseley (1963) beschrieben in einer frühen Arbeit neun Suizidenten, die Suizid begingen mit ihrem Auto als Suizidwaffe. Die Autoren konnten zeigen, dass alle neun Suizidfahrer vor ihrem Autosuizid an einer schweren akuten oder chronischen affektiven Störung gelitten hatten. Ein Jahr später untersuchte MacDonald (1964) 40 Psychiatriepatienten, die einen Suizidversuch überlebt hatten, 30 davon einen versuchten Autosuizid (Single Car Crash). Bei jeder dieser 40 Personen (und somit auch bei den 30 Autofahrern, die einen Suizidversuch mit dem Auto überlebt hatten) konnte das Vorliegen einer schweren psychischen Erkrankung im Vorfeld des Suizidversuchs nachgewiesen werden. Auch Hamburger (1969) untersuchte 41 Psychiatriepatienten nach einem schweren Suizidversuch (22 Frauen, 19 Männer). Sechs der 41 Personen hatten einen Suizidversuch mit dem Auto begangen (fünf Männer, eine Frau). Die Untersucher fanden auch hier, dass bei allen Suizidenten im Vorfeld eine schwere psychische Erkrankung vorgelegen hatte. Zusätzlich hatte es bei jedem der Suizidenten im Vorfeld ihres Suizidversuchs noch zusätzlich ein gravierendes Verlusterlebnis (Partnerverlust, finanzieller Ruin) gegeben. Außerdem hatten drei der sechs Autosuizidenten ein schwerwiegendes Alkoholproblem. Alle sechs Autosuizidenten gaben in dieser Untersuchung an, dass sie bewusst den Suizid mit dem Auto als Todesart gewählt hatten, um ihr Ableben wie einen Unfalltod im Straßenverkehr aussehen zu lassen. Zum einen hätten sie damit ihre Familie vor dem Stigma bewahren wollen, einen „Selbstmörder" in der Familie zu haben - zum anderen hätten sie durch ihren (vermeintlichen) Unfalltod ihre Angehörigen durch Auszahlung der Lebensversicherungssumme auf ihren Namen finanziell sicherstellen wollen.

Mit der von Shneidman (Shneidman 1966, zitiert in Pokorny et al.1972) entwickelten forensischen Untersuchungsmethode der psychologischen Autopsie war es allerdings schon damals möglich, tatsächliche Verkehrsunfallopfer von „erfolgreichen“ Autosuizidenten sicher zu unterscheiden. Auch Pokorny et al. (1972) konnten in ihrer Arbeit mit der Methode der psychologischen Autopsie nach Shneidman unter 28 Verkehrstoten vier eindeutige Fälle von Autosuizid verifizieren. Es sei an dieser Stelle ein kurzer Einschub zur Erklärung der Bedeutung dieser Methode gestattet: Edwin S. Shneidman (1918 - 2009), Begründer der modernen Suizidforschung in den USA, entwickelte mit der von ihm so benannten Methode der psychologischen Autopsie ein psychologisches Untersuchungsverfahren, das es ermöglicht, die äußeren und inneren Umstände zu rekonstruieren, die sich vor dem 
Suizidtod einer Person sehr wahrscheinlich abgespielt haben. Die psychologische Autopsie kann neben der Autopsie des Körpers eines Unfalltoten entscheidende Hinweise zur tatsächlichen Todesursache geben. Durch die Befragung von Familienangehörigen, Freunden und anderen Personen, die mit dem Betroffenen vor seinem Tod Kontakt hatten, durch die Untersuchung von persönlichen Dokumenten und medizinischen Daten des Toten sowie durch die Auswertung des Polizeiberichts über den vermeintlichen Unfalltod im Straßenverkehr kann mit Hilfe der psychologischen Autopsie rekonstruiert werden, was die zu Tode gekommene Person vor ihrem Tod dachte, fühlte und welche Entscheidungen sie aus welchem Grund traf. Zur Frage der Häufigkeit des Auftretens von Autosuizid in Deutschland untersuchte Petersen (1974) im Rahmen seiner Dissertationsarbeit über einen 10-Jahreszeitraum hinweg Fälle von möglichem Autosuizid unter den Straßenverkehrsopfern im Bundesland Schleswig-Holstein. In der Polizeistatistik des Landes fand der Autor im Untersuchungszeitraum 31 Fälle von eindeutigem Autosuizid und vermutete gleichzeitig, dass das möglicherweise noch um weitere Fälle handeln könnte, die er nicht habe verifizieren könne. Petersen begründete seine Vermutung damit, dass nicht selten die polizeilichen Ermittler am Unfallort nach einem schweren Verkehrsunfall mit tödlichem Ausgang ein mögliches Vorliegen von einem Autosuizid gar nicht erst in Betracht ziehen und daher nicht auch in diese Richtung ermitteln würden.

Dieser Ansicht waren seinerzeit auch Tabachnik et al. (1973), die in ihrer Untersuchung der Frage nachgingen, wodurch sich schwere Verkehrsunfälle mit tödlichem Ausgang von einem mit dem Auto durchgeführten Suizid unterscheiden lassen. Sie prägten in diesem Zusammenhang den Begriff der accident prone personality und beschrieben damit Persönlichkeitsauffälligkeiten bei Autofahrern, die durch ein hohes Maß an Depressivität, chronischer Wut, latenter Suizidalität und einer zusätzlichen Suchtproblematik gekennzeichnet sind. Tabachnik et al. kamen zu dem Ergebnis, dass eine nicht unerhebliche Zahl schwerer Verkehrsunfälle mit tödlichem Ausgang gar keine Unfallereignisse im eigentlichen Sinne darstellen, sondern stattdessen eine Suizidhandlung, durchgeführt mit dem Auto im öffentlichen Straßenverkehr. Dazu untersuchten Schmidt et al. (1972) ein Jahr lang im Gebiet Baltimore County schwere Verkehrsunfälle mit tödlichem Ausgang unter der Fragestellung, ob sich unter den Unfallopfern auch bisher nicht erkannte Fälle von Autosuizid befinden könnten. Die Autoren unterteilten die tödlichen Verkehrsunfälle in zwei Gruppen, die sie single car facilities (SC) nannten, wenn nur ein Fahrzeug am Unfall beteiligt war, bzw. multiple car facilities (MC), wenn es mehrere Fahrzeuge als Unfall- 
beteiligte gab. Die Informationen über die Verkehrstoten wurden mit der Methode der psychologischen Autopsie nach Shneidman untersucht und im Anschluss die Fälle beider Gruppen miteinander verglichen. Die tödlich verunglückten Autofahrer der SC- und der MC-Gruppe zeigten dabei deutliche Unterschiede: Die SC-Fahrer $(n=22$, davon 20 Männer und zwei Frauen) waren zum Zeitpunkt ihres Todes jung und alleinstehend und waren der Polizei schon vor ihrem Unfalltod durch riskantes Fahrverhalten und Fahren mit überhöhter Geschwindigkeit aufgefallen. Sie hatten zuvor bereits entsprechende Verkehrsdelikte begangen und hatten bei ihrer Todesfahrt alkoholisiert am Steuer gesessen. Durch Anwendung der psychologischen Autopsie fanden die Untersucher außerdem heraus, dass $70 \%$ der SC-Fahrer psychisch auffällig gewesen waren: Es handelte sich bei ihnen um Personen mit vermehrter Impulsivität, Depressivität sowie mit Alkoholabusus. Bei einem Drittel dieser Fahrer war zuvor bereits eine psychiatrische Diagnose gestellt worden. Die Autoren konnten weiterhin nachweisen, dass es sich bei zwei der 22 tödlich verunfallten SC-Fahrer eindeutig um Autosuizid gehandelt hat. Im Gegensatz zu den SC-Fahrern waren die 11 tödlich verunglückten Fahrer der MC-Gruppe männlich und verheiratet. Drei von ihnen waren im Vorfeld bereits einmal psychisch auffällig geworden. Bei keinem der MCFahrer fanden sich Hinweise für einen Autosuizid als Todesursache. In einer weiteren Studie untersuchte dieselbe Forschergruppe einige Jahre später (Schmidt et al. 1977) noch einmal SC-Fahrer, wieder unter der Fragestellung, ob ihr tödlicher Autounfall in Wahrheit ein Autosuizid gewesen sein könnte. Mit einer größeren Fallzahl $(n=182)$ und über einen Untersuchungszeitraum von sechs Jahren im selben Untersuchungsareal fanden die Autoren jetzt nur drei Fälle von eindeutig gesichertem Autosuizid. Das Durchschnittsalter dieser drei männlichen Suizidenten lag bei 35 Jahren. Mit Hilfe der psychologischen Autopsie konnte festgestellt werden, dass bei allen drei Personen bereits vor dem tödlichen Verkehrsereignis Suizidalität bestand.

Der Frage, ob es beim Autosuizid einen ähnlichen Nachahmer-Effekt (Werther-Effekt) wie bei anderen Suizidarten gibt, ging Phillips (1977) nach und konnte diesen Effekt auch bei Autosuizid nachweisen. Der Autor ging dabei von der Überlegung aus, dass es nach Zeitungsberichten über Suizide bekannter Persönlichkeiten regelhaft zu Nachahmer-Suiziden kommt. Da sich nach Meinung des Autors schwere Verkehrsunfälle nicht selten auch auf eine suizidale Komponente beim Unfallverursacher zurückführen lassen, müsste es daher zeitnah nach einem Zeitungsbericht über einen Prominenten - Suizid zu einer (kurzzeitigen) Zunahme des Auftretens schwerer Verkehrsunfälle kommen. Einen solchen Zusam- 
menhang nachzuweisen gelang dem Autor, indem er nach einem Bericht über den Suizid eines Prominenten in den Medien einen anschließenden kurzzeitigen, aber signifikanten Anstieg des Auftretens schwerer Verkehrsunfälle mit tödlichem Ausgang nachweisen konnte. Einen ganz anderen Ansatz verfolgten Isherwood et al. (1982), die sich in ihrer Untersuchung mit der Bedeutung autodestruktiven Verhaltens in Zusammenhang mit schweren Verkehrsunfällen mit tödlichem Ausgang und Autosuizid beschäftigten. Sie untersuchten in ihrer Studie eine Gruppe von Autofahrern $(\mathrm{n}=100)$, die einen Suizidversuch mit dem Auto überlebt hatten. Im Ergebnis konnte gezeigt werden, dass mehr als $60 \%$ der überlebenden Autosuizidenten zum Zeitpunkt ihres Suizidversuchs manifest psychisch krank waren: Von diesen bestand bei mehr als der Hälfte eine schwere depressive Episode mit akuter Suizidalität, bei den übrigen knapp 40 \% bestand eine akute suizidale Krise bei schwerer Persönlichkeitsstörung. Die Hälfte der überlebenden Autosuizidenten hatten vor Fahrtantritt erhebliche Mengen Alkohol zu sich genommen. Imajo (1983) konnte in einer kleinen Studie von fünf Fällen von vollendetem Autosuizid nachweisen, dass in jedem dieser fünf Fälle im Vorfeld ein gravierendes Lebensproblem bestanden hatte, dass von jedem der Suizidenten als ausweglose Situation erlebt worden war. Bei diesen life events hatte es sich in einem Fall darum gehandelt, dass dem späteren Suizidenten mitgeteilt worden war, dass bei ihm eine schwere körperliche Erkrankung mit infauster Prognose (Lungenkarzinom mit Hirnmetastasen) vorliegt. In einem weiteren Fall war es die Diagnose einer lebensverändernden psychischen Erkrankung (Schizophrenie), im dritten Fall um die Auswirkungen eines schwerwiegenden Alkoholproblems, in den beiden letzten waren soziale Verlustsituationen (Tod des Partner, erhebliche finanzielle Verluste) Auslöser der Suizidhandlung.

In ihrer weitaus größeren Studie kamen Keskinen und Pasanen (1990) zu ähnlichen Ergebnissen: Ebenfalls mit Hilfe der Methode der psychologischer Autopsie untersuchten sie die genaueren Todesumstände und die vorherige persönliche Situation von 937 Verkehrsunfallopfern, die in der staatlichen finnischen Verkehrsunfallstatistik geführt wurden. Unter diesen Verkehrstoten fanden die Untersucher 34 Fälle von eindeutigem Autosuizid (3,63\%). Von diesen 34 eindeutig identifizierten Autosuizidenten hatten 20 (ca. $60 \%$ ) vor ihrer Suizidfahrt nachweislich schwerwiegende psychische Probleme im Sinne von akuter Suizidalität im Rahmen einer schweren depressiven Episode oder als akute suizidale Krise nach einem schwerwiegenden und lebensverändernden Verlustereignis. Auch Kuroda und Pounder (1994), die die genaueren Todesumstände von 268 Verkehrsunfallopfern auf schottischen Straßen untersuchten, kamen zu vergleichbaren Ergebnissen: Unter den 
268 Unfallopfern fanden sie vier gesicherte Fälle von Autosuizid (1,49\%). Bei drei der vier Autosuizidenten hatte vor ihrer Tat eine schwerwiegende Erkrankung vorgelegen (in einem Fall Schizophrenie, in einem weiteren Fall eine schwere Depression und in einem dritten Fall eine fortgeschrittene Tumorerkrankung mit Lungenmetastasen). Harbort (1995), der sich in seiner Arbeit mit dem Phänomen Autosuizid aus verkehrskriminalistischer Sicht beschäftigte, spricht davon, dass Autosuizide einen Anteil von etwa fünf Prozent aller Verkehrsunfälle mit tödlichem Ausgang in Deutschland ausmachen, Ohberg et al. (1997) hingegen kommen bei ihren Untersuchungen auf einen Anteil von 5,9 \%. Zusammenfassend lässt sich sagen, dass bei allen Autofahrern, die mit ihrem Fahrzeug als Tatwaffe im Straßenverkehr Suizid begingen, eine schwere psychische oder somatische Erkrankung vorlag oder ein gravierendes Verlustereignis bei ihnen zu einer suizidalen Krise geführt hat (Ford und Moseley 1963, MacDonald 1964, Hamburger 1969, Keskinen und Pasanen 1990). Bei den psychischen Erkrankungen handelte es sich zumeist um eine schwere depressive Episode mit akuter Suizidalität, eine akute suizidale Krise bei schwerer Persönlichkeitsstörung oder eine schwere Belastungsreaktion mit akuter Suizidalität, ausgelöst durch ein gravierendes Verlusterlebnis (life event). In nicht wenigen Fällen spielten Alkoholprobleme eine zusätzliche Rolle (Tabachnik et al. 1973, Schmidt et al. 1972 und 1977). Der Anteil von Autosuiziden bei schweren Verkehrsunfällen mit tödlichem Ausgang streut in den Literaturangaben zwischen 1,5 - 5,9 \% (Schmidt et al. 1977, Keskinen und Pasanen 1990, Kuroda und Pounder 1994, Harbort 1995). Ohberg et al. 1997).

\subsection{Geisterfahrer/Falschfahrer im Straßenverkehr}

Die Begriffe Geisterfahrer und Falschfahrer werden sowohl in Presseberichten als auch in der Literatur synonym verwendet: Den Begriff Geisterfahrer findet man in Presseberichten jedoch häufiger, da er den emotionalen Aspekt dieses Phänomens im Erleben der anderen Verkehrsteilnehmer widerspiegelt: Plötzlich, geradezu geisterhaft und „wie aus dem Nichts“ kommt dem Autofahrer auf seiner Spur ein anderes Auto entgegengefahren. Das Entsetzen packt ihn, denn er weiß nicht, wie er lebend aus dieser Situation herauskommen soll - der Albtraum eines jeden Autofahrers. Der Begriff Falschfahrer (engl. wrong way driver) dagegen beschreibt den Tatbestand, von dem hier die Rede ist, viel konkreter und sachlicher: Der Begriff Falschfahrer bezeichnet einen Straftatbestand im Verkehrsstrafrecht. Als Falschfahrer gilt ein motorisierter Verkehrsteilnehmer, der das Rechtsfahrgebot 
missachtet, im öffentlichen Straßenverkehr entgegen der Richtungsfahrbahn fährt und dadurch andere Verkehrsteilnehmer erheblich gefährdet. Über das Phänomen „Falschfahrer auf deutschen Autobahnen“ publizierten erstmals die Verkehrspsychologen Färber und Färber (1980). Sie unterteilten in ihrer Untersuchung Falschfahrer in bewusste Falschfahrer (d.h. die bewusst und absichtlich entgegen der Richtungsfahrbahn unterwegs sind) und nicht bewusste Falschfahrer (solche, die irrtümlich zu Geisterfahrern werden und dann vor Angst wie gelähmt sind, wenn sie ihren folgenschweren Irrtum bemerken). Auch Bierwas et al. (1981) unterschieden in ihrer Arbeit zwischen absichtlichen und unabsichtlichen Falschfahrten. Hierzu hatten beide Untersuchergruppen Falschfahrermeldungen der Polizei der Jahre 1978 und 1979 ausgewertet und waren am Ende zu ähnlichen Ergebnissen gelangt: Lediglich sieben Prozent der Falschfahrer, über die der Verkehrsfunk eine Falschfahrerwarnung ausgibt, verursachen tatsächlich einen schweren Verkehrsunfall. Die übergroße Mehrheit der Falschfahrer dieser Studie wurde rechtzeitig von der Polizei gestoppt oder erkannten ihren Irrtum selbst und verließen umgehend die Autobahn. In den Fällen, in denen es jedoch zu einem Frontalzusammenstoß zwischen einem Falschfahrer mit einem Gegenfahrzeug kam, handelte es sich stets um einen außerordentlich schweren Unfall mit Toten, Schwerverletzten und hohen Sachschäden.

Konkrete Zahlen liefern hierzu Bierwas et al. (1981): Bei den von ihnen untersuchten 100 Geisterfahrer-Unfällen starben insgesamt 37 Verkehrsteilnehmer. In dieser Untersuchung fanden sich zwei Gruppen von Autofahrern, die überdurchschnittlich häufig GeisterfahrerUnfälle verursachten: Zum einen waren dies männliche Autofahrer zwischen 25 - 44 Jahren, zum anderen ältere Autofahrer über 65 Jahre. Ein Drittel der von ihnen untersuchten Falschfahrer hatte alkoholisiert am Steuer gesessen, $16 \%$ davon sogar erheblich alkoholisiert. Als häufigste Ursache einer absichtlichen Falschfahrt wurden von diesen Autoren waghalsige Wendemanöver auf der Autobahn genannt, die in der Absicht durchgeführt wurden, einen früheren Richtungsfahrfehler zu korrigieren. Als häufigste Ursache einer unabsichtlichen Falschfahrt nannten die Autoren die unabsichtliche falsche Richtungsentscheidung eines Autofahrers infolge fehlender örtlicher Orientierung. Als Ursachen des Orientierungsverlustes wurden genannt: Ortsunkenntnis des Autofahrers, widrige Witterungsumstände wie dichter Nebel, unübersichtliche und missverständliche Ausschilderung von Autobahnauffahrten. Ellinghaus und Steinbrecher (1994) konnten belegen, dass die Hälfte aller Falschfahrten absichtliche Falschfahrten darstellen, die auf eine hochriskante 
Entscheidung eines Autofahrers (wie z. B. ein Wendemanöver auf der Autobahn zur Korrektur eines vorherigen Richtungsfahrfehlers) zurückgeführt werden können. Bei den Verursachern unabsichtlicher Falschfahrten machten Autofahrer unter Alkoholeinfluss mit $25 \%$ den größten Anteil aus. Diese Zahl deckt sich mit den verkehrsstatistischen Erhebungen des Bundesamtes für Straßenwesen (2003) über polizeilich registrierte alkoholisierte Falschfahrer der Jahre 1990-1993. Wie schon zuvor Bierwas et al. (1981) kamen auch Ellinghaus und Steinbrecher (1994) zu einem ähnlichen Ergebnis: Nur 6 - 8 \% aller Geisterfahrten enden mit einem schweren Verkehrsunfall mit Toten, Verletzten und erheblichen Sachschäden.

Einen ganz anderen Untersuchungsansatz verfolgten Scaramuzza und Cavegn (2006) in ihrer Studie über Geisterfahrer: Über einen Zeitraum von zwei Jahren werteten sie Geisterfahrer-Meldungen des Schweizer Verkehrssenders Viasuiss aus, die sie mit der Häufigkeit des Auftretens von Geisterfahrer-Unfällen in dem von ihnen untersuchten Gebiet (Autobahnnetz der Schweiz) verglichen. Die Autoren kamen zu dem Ergebnis, dass nur 0,3 \% aller schweren Autobahnunfälle mit Personenschäden im Untersuchungszeitraum von Geisterfahrern verursacht worden waren. Allerdings waren diese Geisterfahrer-Unfälle mit einer 7,5fach erhöhten case fatility auch die schwersten Autobahnunfälle überhaupt. Des Weiteren stellten die Untersucher fest, dass zwei Drittel der Geisterfahrer Männer waren, ein Drittel von ihnen war während ihrer Geisterfahrt alkohol- und/oder Drogen intoxikiert. Eine eindeutige Selbsttötungsabsicht konnte nur bei 0,9\% der untersuchten Geisterfahrerunfälle nachgewiesen werden.

Die bisher größte Studie über Geisterfahrer auf bundesdeutschen Autobahnen ist die ADAC-Studie über Falschfahrer (Berlitz 2012): In dieser Studie wurden über einen Zeitraum von sechs Jahren (2005 - 2011) sämtliche Polizeimeldungen über Falschfahrer auf bundesdeutschen Autobahnen gesammelt und ausgewertet. Der Autor fand über den gesamten Untersuchungszeitraum hinweg einen stabilen Wert von etwa 1.800 - 1.900 Falschfahrermeldungen pro Jahr bezogen auf das gesamte Bundesgebiet. Des Weiteren zeigte die Studie, dass Falschfahrten auf verschiedenen Autobahnabschnitten unterschiedlich häufig vorkommen: Ein überdurchschnittliches Falschfahrer-Aufkommen wurde auf Autobahnabschnitten mit großer Verkehrs- und Anschlussstellendichte festgestellt - und da besonders im Autobahnnetz von Großstädten wie Berlin und Hamburg sowie im Rhein-RuhrBallungsraum. Die Zahl der Falschfahrermeldungen von den Autobahnabschnitten auf dem 
Gebiet der neuen Bundesländer lag dagegen um 25 - $50 \%$ unter dem Bundesdurchschnitt. Berlitz (2012) führte dieses Phänomen in erster Linie auf die straßenbaulichen Gegebenheiten zurück: Sämtliche Autobahnabschnitte auf dem Gebiet der ehemaligen DDR seien in den 90er Jahren grundlegend saniert und nach modernen verkehrswissenschaftlichen Erkenntnissen umgestaltet worden - einschließlich einer deutlich verbesserten Ausschilderung der Autobahnauffahrten (persönliche Mitteilung am Telefon).

Ohne Angabe von Häufigkeiten nennt die Studie als Ursachen von Falschfahrten: 1) Falschfahrten junger Männer in Alkohol und/oder Drogen intoxikiertem Zustand, 2) Fahrten entgegen der Fahrtrichtung in suizidaler Absicht (Suizid mit dem Auto), 3) Panikreaktionen von Autofahrern (z. B. Flucht vor der Polizei mit dem Auto), 4) Falschfahrten junger Autofahrer als sogenannte „Mutprobe“, 5) Wenden auf freier Strecke zur Korrektur eines früheren Richtungsfahrfehlers, 6) falsches Auffassen einer Aufforderung aus dem Navigationsgerät, 7) Orientierungsverlust an einer unübersichtlichen Anschlussstelle, 8) widrige Witterungsbedingungen wie dichter Nebel, 9) ortsunkundiger, mit der Situation überforderter Autofahrer und 10) Fehler eines altersverwirrten Autofahrers. 
Über Persönlichkeitsbesonderheiten von Geisterfahrern publizierten Schützhofer et al. (2008): Die Autoren werteten dazu eine von Hitradio Ö3 initiierte Online-Hörerbefragung $\mathrm{zu}$ einer möglichen eigenen Geisterfahrt in den letzten 3 Jahren aus. Die 867 Ö3Radiohörer, die sich an dieser Umfrage beteiligten (473 Männern, 394 Frauen, Durchschnittsalter 27 Jahre), wurden gebeten, anonym und online einen Fragebogen auszufüllen. Dieser von Schützhofer et al (2008) selbst erstellte Fragebogen erfasste die Persönlichkeitsdimensionen: Emotionale Stabilität, Selbstkontrolle, soziales Verantwortungsbewusstsein und Abenteuerlust/Spannungs-bedürfnis.

Die Auswertung der eingesandten Fragebögen ergab, dass 16 Teilnehmer dieser Umfrage innerhalb der letzten drei Jahre wenigstens einmal als Geisterfahrer auf öffentlichen Straßen unterwegs gewesen sein mussten. Weiterhin zeigten die Ergebnisse der OnlineBefragung, dass 7 der 16 ermittelten Geisterfahrer absichtlich und in Alkohol- u./o. Drogen intoxikiertem Zustand entgegen der Richtungsfahrbahn unterwegs gewesen waren. Diese hatten zuvor schon andere Verkehrsdelikte begangen. Ihre Falschfahrt war Teil eines nächtliches „Freizeit-Events“ von jungen Männern. Im Ergebnis der Studie wurden bei diesen intentionalen Geisterfahrern folgende Persönlichkeitseigenschaften signifikant häufiger anzutreffen als bei den Personen der Vergleichsgruppe: Ein hohes Maß an emotionaler Instabilität und verminderter Selbstkontrolle, eine geringe innere Bereitschaft, sich an allgemeingültige Regeln und Normen zu halten (die bei den betreffenden Personen auch weniger verinnerlicht erschienen als bei den Personen der Vergleichsgruppe), eine geringe Anstrengungsbereitschaft und ein orientiert sein an leicht erreichbaren Zielen, ein signifikant niedrigeres Verantwortungsbewusstsein sowie eine signifikant geringere Einsicht in die zu erwartenden negativen Konsequenzen ihres Fehl- bzw. Straftatverhaltens. Interessanterweise fand sich bei den Persönlichkeitsdimensionen „Abenteuerlust“ und „Spannungsbedürfnis“ kein signifikanter Unterschied zwischen den intentionalen Geisterfahrern und den Personen der Vergleichsgruppe. Schützhofer et al. (2008) kamen zu dem Ergebnis, dass es sich bei den absichtlichen Geisterfahrern dieser Untersuchung um eine relativ kleine Gruppe junger männlicher Autofahrer handelte (0,81\% der Gesamtstichprobe), denen es schwerfällt, Regeln zu akzeptieren und zu verinnerlichen, sich an diese zu halten und die Folgen einer Regelübertretung vorab zu antizipieren.

Zusammenfassend ist festzustellen, dass in der Literatur Geisterfahrten bzw. Falschfahrten in intentionale und nicht intentionale unterteilt werden (Färber und Färber 1980, Bierwas et 
al. 1981). Auf bundesdeutschen Autobahnen werden seit Jahren stabil etwa 1800 - 1900 Geisterfahrten jährlich registriert (ADAC-Studie, Berlitz 2012). Jedoch verursachen nur $5-8 \%$ der polizeilich registrierten und über Verkehrsfunk gemeldeten Geisterfahrer tatsächlich einen schweren Verkehrsunfall (Bierwas et al. 1981, Ellinghaus und Steinbrecher 1994, Bundesamt für Straßenwesen 2003). Es handelt sich bei Geisterfahrerunfällen aber

immer um katastrophale Verkehrsunfälle mit Toten, Verletzten und hohen Sachschäden (Bierwas et al. 1981, Ellinghaus und Steinbrecher 1994, Scaramuzza und Cavegn 2006). Falschfahrer sind zumeist männlich, 25 - 44 Jahre alt (erster Altersgipfel) bzw. älter als 65 Jahre (zweiter Altersgipfel) (Bierwas et al. 1981, Scaramuzza und Cavegn 2006, Schützhofer et al. 2008). 30 - 50 \% der Falschfahrer sind während ihrer Falschfahrt alkoholisiert (Bierwas et al. 1981, Schützhofer et al. 2008). Junge männliche Falschfahrer haben eine typische Charakterpathologie (Schützhofer et al. 2008).

\subsection{Die Amokfahrt als Sonderform von Amok im Straßenverkehr}

Das Phänomen des Amokfahrers stellt eine sehr selten vorkommende Sonderform des Amokläufers dar (Adler 2010a). Eine Amokfahrt ist somit eine Amoktat, bei der nicht wie beim Amokläufer eine Schuss-, Schlag- oder Stichwaffe benutzt wird, um die Tat auszuführen, sondern eine atypische Waffe wie ein motorisiertes Fahrzeug. In den meisten Fällen verwendeten Amokfahrer zur Ausführung einer Amokfahrt einen Pkw oder Lkw, seltener einen Panzer oder ein schweres Baufahrzeug, ganz selten ein Flugzeug (Adler et al. 1993, Adler 2006, Adler 2008, Adler 2010a, Adler 2010b, Adler 2015a, Adler 2015b). Im Spektrum der Tötungsdelikte mit Mord und Totschlag als Homizidtaten an einem Ende und der Selbsttötung (Suizid) am anderen Ende des Spektrums nehmen Amoklauf und Amokfahrt als Homizid-Suizid-Taten in diesem Spektrum eine Mittelposition ein, da sie die Kriterien der Menninger-Trias erfüllen (Menninger 1938): Sie haben bei ihrer Amoktat 1) sowohl die Intention zu töten (Homizid-Anteil der Tat), zum anderen aber 2) auch die Intention, sich am Ende ihrer Tat selbst zu töten (Suizid-Anteil der Tat), bzw. 3) kann es auch die Intention sein, ihren Tod auf passive Weise zu suchen: Da kann am Ende ihrer Flucht sein, wenn sie sich der Polizei nicht ergeben, sondern sich bewaffnet gegen die Polizei wenden und dann ggf. von Spezialkräften erschossen werden. Die früheste bekannte Untersuchung über Homizid-Suizid-Taten stammt von Wolfgang (1958). Mit seiner weapon substitution 
hypothesis postulierte der Autor, dass die Absicht des Täters (zu verletzen oder zu töten) bereits an der Wahl seiner Tatwaffe erkennbar ist. Die Tatwaffen, die heutige Amoktäter verwenden, unterscheiden sich deutlich von denen, die der historische malaysische Amokläufer verwendete (Adler 2008, Adler 2015a). Heutige Amoktäter verwenden überwiegend Schusswaffen, seltener Stichwaffen. Und noch viel seltener kommt eine atypische Waffe wie ein motorisiertes Fahrzeug für eine Amokfahrt zum Einsatz (Adler 2015a). Im Vergleich zur statistischen Häufigkeit des Auftretens von Amokläufen (ein Fall auf 6,7 Mio. Männerjahre) kommen Amokfahrten im öffentlichen Straßenverkehr 10mal seltener vor und stellen somit außerordentlich seltene Ereignisse dar (Adler 2015b). Die Psychopathologie von Amoktätern ist vielfach beschrieben worden (Adler et al. 1993, Adler et al. 1994, Adler 2001, Adler 2006, Adler et al. 2006, Adler 2010a, Adler 2010b, Adler 2015a Adler 2015b). Amoktäter sind in ihrer überwiegenden Zahl männlich, weibliche Amokläufer oder Amokfahrer stellen eine außerordentliche Rarität dar.

Nach allem was bislang über die Persönlichkeitsbesonderheiten von Amoktätern bekannt ist, sind sie in den allermeisten Fällen Einzelgänger, haben nur sehr wenige soziale Kontakte und leben zurückgezogen. Ihre Art zu denken und $\mathrm{zu}$ handeln ist rigide und wenig anpassungsfähig, hervortretende Persönlichkeitsmerkmale bei ihnen sind übermäßige Kränkbarkeit und Rachsucht nach Niederlagen. Dadurch sind sie nur sehr eingeschränkt in der Lage, auf angemessene Art und Weise mit den ganz normalen lebenstypischen Kränkungen fertig zu werden. Dazu kommt noch eine übermäßige Impulsivität, durch die es bei diesen Personen häufig zu einem jähzornigen Ausagieren von Alltagskonflikten kommt. Adler (2010a) konnte zeigen, dass bei späteren Amoktätern psychiatrische Erkrankungen im Vergleich zum Bevölkerungsdurchschnitt deutlich überrepräsentiert sind. Wahnkranke gelten als die Gefährlichsten unter den Amoktätern (Adler et al. 1993). 
Tabelle 1: Vergleich Amokläufer und Amokfahrer

Amokläufer

\begin{tabular}{|c|c|c|}
\hline Geschlecht & $95 \%$ männlich & 95,7 \% männlich \\
\hline Alter & $34,8+/-12,1$ Jahre & 35,5 Jahre \\
\hline Familienstand & $\begin{array}{l}60 \% \text { in Lebensgemeinschaft } \\
40 \% \text { alleinlebend }\end{array}$ & $\begin{array}{l}35 \% \text { verheiratet o. Lebensgemeinschaft, } \\
26 \% \text { alleinlebend, } 39 \% \text { unbekannt }\end{array}$ \\
\hline Beruf & $\begin{array}{l}12 \% \text { Akademiker, } 21 \% \text { Angestellte, } \\
50 \% \text { Arbeiter, } 5 \% \text { Studenten, } \\
8 \% \text { ohne Beruf }\end{array}$ & $\begin{array}{l}\text { 8,7 \% Akademiker, } 13 \% \text { Angestellte, } 21,7 \% \\
\text { Arbeiter, 56,6\% keine Angaben }\end{array}$ \\
\hline auslösende Motive & $\begin{array}{l}\text { überwiegend Trennungs-, Verlust- } \\
\text { und Konfliktsituationen, selten } \\
\text { wahnhafte Motive }\end{array}$ & $\begin{array}{l}\text { Arbeits- oder Wohnungsverlust, Niederlage vor } \\
\text { Gericht (aber: in } 39 \% \text { der Fälle keine Angaben } \\
\text { hierzu vorliegend) }\end{array}$ \\
\hline $\begin{array}{l}\text { Handlungslatenz } \\
\text { Phasenmodell }\end{array}$ & $\begin{array}{l}\text { Amok-4-Phasen-Ablauf fast } \\
\text { immer nachweisbar }\end{array}$ & $\begin{array}{l}\text { nur bei } 10 \% \text { der Amokfahrten } \\
\text { Amok-4-Phasenmodell nachweisbar, } \\
\text { bei } 90 \% \text { Tatbeginn unmittelbar nach tatauslö- } \\
\text { sendem Ereignis }\end{array}$ \\
\hline $\begin{array}{l}\text { Täter-Opfer - } \\
\text { Beziehung }\end{array}$ & $\begin{array}{l}\text { anfangs nahe Täter-Opfer- } \\
\text { Beziehung (Opfer Verwandte, Be- } \\
\text { kannte, Konfliktpartner), sekundäre } \\
\text { Ausweitung der Tat auf Unbekann- } \\
\text { te }\end{array}$ & $\begin{array}{l}\text { 73,9 \% Opferwahl zufällig, } \\
13 \% \text { Täter-Opfer-Beziehung, } \\
13 \% \text { Täter-Opfer-Beziehung und sekundäre } \\
\text { Ausweitung der Tat auf Unbekannte }\end{array}$ \\
\hline $\begin{array}{l}\text { Primärpersönliche } \\
\text { Auffälligkeiten }\end{array}$ & $\begin{array}{l}\text { gehemmt-aggressiv, kontaktscheu, } \\
\text { sexuell abstinent, querulatorisch }\end{array}$ & $\begin{array}{l}\text { impulsiv und unbeherrscht, querulatorisch, } \\
\text { hypochondrisch, paranoid-Wahn nah }\end{array}$ \\
\hline $\begin{array}{l}\text { Psychiatrische } \\
\text { Erkrankung vor der } \\
\text { Tat }\end{array}$ & $\begin{array}{l}20 \% \text { Intoxikation (exogene } \\
\text { Psychose), } 17 \text { \% Psychose, } \\
15 \% \text { Persönlichkeitsstörung (über- } \\
\text { wiegend vom impulsiven Typ), } \\
5 \% \text { Wahn, Rest unbekannt }\end{array}$ & $\begin{array}{l}13 \% \text { akute Intoxikation, } \\
26,1 \% \text { Affekthandlung nach akuter psychischer } \\
\text { Belastung, } 35 \% \text { Psychosen, 8,7 \% Wahn, } \\
\text { 8,7 \% schwere Persönlichkeitsstörung, Rest } \\
\text { unbekannt }\end{array}$ \\
\hline Tat-Ende & $\begin{array}{l}35 \% \text { Tod des Täters durch Suizid, } \\
6,6 \% \text { Tod durch Fremdeinwirkung, } \\
\text { z. B. Polizei ( } 41,6 \% \text { gesamt), } \\
11 \% \text { widerstandslose Festnahme, } \\
\text { Rest unbekannt }\end{array}$ & $\begin{array}{l}\text { nur in } 1 \text { Fall (8,7 \%) Tod des Amokfahrers, } \\
\text { 91,3 \% der Amokfahrer überleben Amokfahrt, } \\
\text { von diesen 82,6\% Fluchtversuch nach der Tat, } \\
\text { davon 43,5 \% Überwältigung durch Polizei, } \\
\text { 43,8 \% Suizidversuch nach der Tat, } \\
\text { 17,4 \% widerstandslose Festnahme }\end{array}$ \\
\hline Opfer / Schäden & $\begin{array}{l}\text { durch Schusswaffengebrauch in } \\
85 \% \text { der Amokläufe überwiegend } \\
\text { Todesopfer, wenig Verletzte, wenig } \\
\text { Sachschäden }\end{array}$ & $\begin{array}{l}\text { bei mehr als } 50 \% \text { der Amokfahrten keine Todes- } \\
\text { opfer, dafür deutlich mehr Verletzte und in } \\
78,3 \% \text { der Fälle erhebliche Sachschäden }\end{array}$ \\
\hline Verurteilung & $\begin{array}{l}\text { in mehr als } 40 \% \text { keine Verurtei- } \\
\text { lung, da Täter selbst verstorben }\end{array}$ & $\begin{array}{l}\text { soweit bekannt (in } 60 \% \text { der Fälle keine Anga- } \\
\text { ben) Verurteilung der schuldfähigen Amokfahrer } \\
\text { zu Haftstrafen ( } 2 \text { - } 7 \text { Jahre), der nicht schuldfähi- } \\
\text { gen psychose- und wahnkranken Amokfahrer } \\
\text { zur Unterbringung in einer Psychiatrischen Klinik } \\
\text { gemäß § } 63 \text { StGB }\end{array}$ \\
\hline
\end{tabular}

Die Daten zu den Amokläufern in Tabelle 1 sind mit freundlicher Genehmigung des Autors, entnommen aus Adler (2000). 
Asberg et al. (1976) konnten zeigen, dass das Ausmaß des impulsiv-aggressiven Verhaltens eines Menschen mit der Liquor-Konzentration des Serotonin-Metaboliten 5HydryIndolsäure (5-HIAA) negativ korreliert. Aus diesem Zusammenhang wurde die Serotoninmangel-Hypothese abgeleitet (Adler 2010b), die ein biologisches Erklärungsmodell für den vollständigen Impulskontrollverlust des Amoktäters unmittelbar vor der Tat im Sinne einer massiven Dekompensation eines bereits vorbestehenden Serotoninmangel-Syndroms liefert (Adler 2008).

Zusammenfassend ist festzustellen, dass die Aussagen in der Amokliteratur getätigten Aussagen über Amokläufer grundsätzlich auch auf Amokfahrer zutreffen. Letztere weisen aber im Vergleich zum Amokläufer weitere Besonderheiten auf, durch die sie sich vom Amokläufer unterscheiden: Während bei den Amokläufern Wahnkranke und Menschen mit einer (akut dekompensierten) schweren Persönlichkeitsstörung häufiger vorkommen, sind unter den Amokfahrern häufiger Psychotiker wie Schizophrenie-Kranke anzutreffen, die spontan und unvorbereitet handeln und zur Begehung ihrer Tat nicht selten eine atypische Waffe wie ein Auto benutzen, wenn sie zufällig in deren Besitz gelangen (Adler et al. 1993).

Alles in allem sind mehr als $85 \%$ aller schweren Verkehrsunfälle mit Personenschäden nicht auf ein Versagen der modernen Technik, sondern auf ein Fehlverhalten von Autofahrern zurückzuführen. Somit hat die Erforschung der Ursachen des Fehlverhaltens von Autofahrern eine nicht zu unterschätzende Bedeutung für die allgemeine Verkehrssicherheit. $\mathrm{Zu}$ den Verkehrsteilnehmern, die überdurchschnittlich häufig schwere Verkehrsunfälle verursachen, zählen somit: Aggressive, junge männliche Autofahrer, Personen, die vorhaben, mit dem Auto im Straßenverkehr Suizid zu begehen, Autofahrer mit einer psychiatrischen und/oder Suchterkrankung in einem akuten psychischen Ausnahmezustand und/oder erheblich Suchtmittel intoxikiert, Falsch- oder Geisterfahrer im Allgemeinen - und die (echten) Amokfahrer im Besonderen. Letztere können in Teilaspekten Verhaltensbesonderheiten der anderen Risikofahrergruppen aufweisen, lassen sich aber mit Hilfe der AmokKriterien eindeutig von diesen abgrenzen. 


\subsection{Fragestellungen und Ausgangshypothese}

Die Fragestellungen, die dieser Untersuchung vorangestellt wurden, lauten: Ist es möglich, durch Auswertung von Zeitungsberichten über schwere Verkehrsunfälle echte Amokfahrer in einem umschriebenen Gebiet und innerhalb eines definierten Zeitraums anhand der Amokkriterien eindeutig zu identifizieren? Ist es des Weiteren möglich, bei den von journalistischer Seite „Amokfahrten“ genannten Verkehrsereignissen echte Amokfahrten von anderen schweren Verkehrsereignissen mit Personenschäden sicher abzugrenzen? Und schließlich: Lässt sich der subjektive Eindruck aus Presseberichten, dass Amokfahrten im Straßenverkehr zunehmend häufiger auftreten, durch die hier erhobenen Daten verifizieren oder falsifizieren?

Die Ausgangshypothese dieser Untersuchung lautet: Die Zahl der echten Amokfahrten auf deutschen Straßen ist nicht angestiegen, sondern bleibt über den gesamten Untersuchungszeitraum hinweg konstant auf sehr niedrigem Niveau.

\section{Material und Methoden}

\subsection{Quellen bei der Materialgewinnung}

Eine Überlegung, wie Daten über Amokfahrten und andere schwere Verkehrsunfälle mit Personenschäden auf dem Gebiet des Freistaats Thüringen im Zeitraum 2001 - $2010 \mathrm{zu}$ gewinnen wären, bestand ganz am Anfang der Untersuchungen darin, Datenmaterial über schwere Verkehrsunfälle aus dem Thüringer Polizeiarchiv auszuwerten. Eine entsprechende Bitte um Genehmigung der Nutzung des Thüringer Polizeiarchivs zur Datengewinnung für den Zweck einer wissenschaftlichen Untersuchung beantwortete das Thüringer Innenministerium jedoch abschlägig mit der Begründung, dass zum einen Schadensereignisse im Straßenverkehr mit möglicherweise suizidalem Hintergrund in der Thüringer Verkehrsunfallstatistik nicht explizit erfasst würden. Zum anderen wurde darauf ausdrücklich darauf 
hingewiesen, dass die von der Thüringer Polizei erhobenen und gespeicherten Daten ausschließlich dem Zweck der polizeilichen Aufgabenerfüllung dienen würden, einem Zweckbindungsgebot unterlägen und deshalb für wissenschaftliche Untersuchungen nicht zur Verfügung stünden. Nach dieser Absage war, was die Datengewinnung für diese Untersuchung betraf, eine grundlegende Neuorientierung erforderlich. Auf Empfehlung ihres Betreuers Professor Adler entschloss sich die Untersucherin, die Datengewinnung auf dem Weg der Presserecherche durchzuführen, analog der Datengewinnung bei seiner DreiDekaden-Amokstudie. Die Gewinnung von Sekundär-Datenmaterial aus Presseberichten hat unbestritten Schwächen, die später noch erörtert werden. Sie erwies sich auf eine gewisse Weise aber auch als Vorteil: So konnten die eigenen Untersuchungsergebnisse mit den Fällen von Amokfahrten der Drei-Dekaden-Studie, die ebenfalls aus Presseberichten gewonnen und kontentanalytisch untersucht worden waren, verglichen und diskutiert werden.

\subsection{Datenlage zu Berichten über Amokfahrten in lokalen Zeitungen des Freistaates Thüringen}

Um Daten für die geplanten Untersuchungen zu gewinnen, sichtete die Untersucherin im Archiv der Zeitungsgruppe WAZ (Westfälische Allgemeine Zeitung) Thüringen, Sitz Erfurt, die Online-Ausgaben sämtlicher Thüringer Tageszeitungen der Jahre 2001 - 2010. Die WAZ Zeitungsgruppe Thüringen gibt drei verschiedene Tageszeitungen heraus, die in unterschiedlichen Regionen des Freistaates Thüringen erscheinen und demzufolge auch unterschiedliche Regionalnachrichten beinhalten. Es handelt sich bei diesen lokalen Tageszeitungen um die Thüringer Allgemeine Zeitung (TA), die in allen Teilen Thüringens gelesen wird und in den verschiedenen Landkreisen mit einer jeweils anderen Regionalbeilage ausgeliefert wird, die Thüringer Landeszeitung (TLZ), die vor allem in den Landkreisen Süd- und Westthüringens ihre Leser hat und die Ostthüringer Zeitung (OTZ), die vor allem in Nord- und Ostthüringen vertreten ist. Im schon erwähnten Online-Archiv der WAZZeitungsgruppe Thüringen wurden von der Untersucherin die Zeitungsjahrgänge 2001 2010 jahrgangsweise in allen drei genannten Tageszeitungen (TA, TLZ, OTZ) nach Artikeln über Amokfahrten durchsucht. Da aber damit gerechnet werden musste, dass Artikel in ihrer Überschrift das Wort Amokfahrt haben könnten, ohne dass bei dem berichteten 
Geschehen um eine echte Amokfahrt entsprechend der Amok-Definition handelt (oder dass möglicherweise in der Überschrift des Artikels das Wort Amokfahrt gar nicht vorkommt, es sich aber bei dem berichteten Vorfall dennoch um eine echte Amokfahrt handeln könnte), erfolgte die Online-Suche breiter gefasst mit Hilfe der Schlagworte: Schwerer Unfall, Autobahn-Tote, Unfallfahrer, Geisterfahrer, Falschfahrer, Alkoholfahrt, Geisterfahrt, Amokfahrt und Amokfahrer.

Die Online-Suche erbrachte aus dem Pool von 10 Jahrgängen dreier Thüringer Tageszeitungen (insgesamt etwa 1000 Ausgaben) 76 Zeitungsartikel über 73 Fälle mit Angaben, die auf eine Amokfahrt hindeuteten. Über einen besonders spektakulären Fall erschienen von der Tat bis zur Verurteilung des Amokfahrers vier Artikel. Im Anschluss daran wurden die Angaben zu den Tatumständen in diesen 76 Presseartikeln mit Hilfe der Amok-Kriterien einer näheren Untersuchung auf ein mögliches Vorliegen einer echten Amokfahrt unterzogen.

\subsection{Datenlage zu Berichten über schwere Verkehrsunfälle durch Amokfahrer in der überregionalen Presse}

Diese Untersuchungen für den Zeitraum Februar 2001 bis Mai 2010 wurden parallel zu den Thüringer Untersuchungen durchgeführt. Durch direkte Internet-Recherche in OnlineAusgaben überregionaler Zeitungen und Zeitschriften sowie Anschreiben lokaler Zeitungsredaktionen mit der Bitte um Zusendung dort veröffentlichter Artikel über Amokfahrten konnte die Untersucherin Vergleichsmaterial aus folgenden Quellen innerhalb der bundesdeutschen Presselandschaft gewinnen (in alphabetischer Reihenfolge):

Aachener Zeitung (AZ), Allgemeine Zeitung Bad Kreuznach (AZB), Auto Motor Sport, Berliner Kurier (BK), Berliner Zeitung (BZ), BILD, Deutsch Presseagentur (DPA), Die Welt (DW), Frankfurter Rundschau (FR), Freiburger Zeitung (FZ), Hamburger Abendblatt (HA), Hamburger Morgenpost (HP), Münchner Abendzeitung (MAZ), Münsterländische Tageszeitung (MZ), Nürnberger Nachrichten $(\mathrm{NN})$, Passauer Neue Presse (PNP), Sächsische Zeitung/Chemnitzer Morgenpost (SZ/ChM), Spiegel Online, Süddeutsche Zeitung (SZ), Weser Kurier (WK), Westdeutsche Allgemeine Zeitung (WAZ). 
Die Materialsuche erfolgte mit Hilfe der Schlagwörter: Schwerer Unfall mit Todesopfern, Todesfahrt, Geisterfahrt, Amokfahrt, Todesfahrer, Geisterfahrer, Falschfahrer und Amokfahrer. Die meisten Treffer erbrachten die Begriffe Amokfahrt und Amokfahrer, wobei es sich aber bei genauerer Prüfung dabei zumeist nicht um echte Amokfahrten handelte. Es wurde deutlich, dass die Überschrift „Amokfahrt“ in den untersuchten Presseberichten von journalistischer Seite auch für andere schwere Verkehrsunfälle mit Personen- und Sachschäden verwendet wurde. Bei der Online-Recherche konnte dankenswerterweise mehrfach auf das Spiegel-Online-Archiv zugegriffen werden. Verwendung fanden außerdem die Portale: www.abendblatt.de, www.abendzeitung-muenchen.de, www.auto-motor-sport.de, www.berliner-kurier.de, www.berliner-zeitung.de, www.derwesten.de, www.express.de, www.fr-online.de, www.nuernberger-nachrichten.de Bereits bei der Online-Durchsicht des jeweiligen Artikels wurde geprüft, ob hier tatsächlich über eine Amokfahrt im Sinne der Amok-Definition berichtet wurde oder über eine journalistisch-sensationsheischend mit dem Etikett Amokfahrt versehene andere Verkehrsstraftat, die aber einer Prüfung anhand der Amok-Kriterien nicht standhielt. Mit Unterstützung der wissenschaftlichen Fachbibliothek des Ökumenischen Hainich Klinikums Mühlhausen/Thür. wurde bei neun der untersuchten Fälle möglicher Amokfahrten schriftlich bei den Redaktionen lokaler Tageszeitungen am Ort des Geschehens um weiteres Datenmaterial in Form nachfolgender Artikel über dieselbe Amokfahrt angefragt - optimaler Weise vom Tag der Tat an bis zur Urteilsverkündung (wenn der Amokfahrer seine Tat überlebt hat). Diese Anfragen waren in allen Fällen erfolgreich. Durch die zugesandten Artikel, die nur in der regionalen Presse erschienen waren, stand nun wesentlich mehr auswertbares Datenmaterial zur Verfügung als nur aus den oft kurzgefassten Online-Artikeln großer überregionaler Zeitungen. Nach umfangreicher Recherche mit Hilfe der genannten Quellen und nach strenger Auswahl entsprechend der wissenschaftlichen Amok-Kriterien konnten deutschlandweit für den Zeitraum 2001 - 201023 Fälle echter Amokfahrten gesichert werden, die in die nachfolgende Auswertung Eingang fanden. 


\subsection{Berichte über Amokfahrer in der deutschen Presse aus dem europäischen Ausland im Untersuchungszeitraum 2001 - 2010}

In diesem Zeitraum berichtete die deutsche Presse über 14 Amokfahrten im europäischen Ausland (Österreich: sechs Fälle, Schweiz: vier Fälle, Frankreich: zwei Fälle, Schweden: ein Fall, Niederlande: ein Fall).

\subsection{Berichte über Amokfahrer in der deutschen Presse aus dem weltweiten Geschehen in den Jahren 2001 - 2010}

Im selben Zeitraum wurde in der deutschen Presse über 10 Amokfahrten berichtet, die sich außerhalb Europas ereignet hatten (USA: vier Fälle, Israel: vier Fälle, Mexiko: ein Fall, China: ein Fall).

Zusammenfassend heißt das, dass nur die Anzahl der deutschlandweiten Fälle (Kap. 2.3) der Dekade III (2001 - 2010) mit 23 Fällen echter Amokfahrten groß genug war, um sie mittels der kontentanalytischen Methode zu kodieren und in der Folge statistisch verwertbare Ergebnisse zu erzielen. Wegen der für eine eigenständige statistische Auswertung zu geringen Thüringer Fallzahlen (Kap. 2.2) gingen die Thüringer Fälle in die gesamtdeutsche Untersuchung mit ein. Die Presseberichte über Amokfahrten der Kap. 2.2 (Thüringen), Kap. 2.4 (europäisches Ausland) und Kap. 2.5 (weltweit) wurden außerdem wegen der jeweils geringen Fallzahlen im Sinne einer Falltypisierung ausgewertet. Zusammen mit den 23 deutschen Amokfahrten (inklusive der zwei gesicherten Thüringer Fälle) wurden im Untersuchungszeitraum zusammen mit den europäischen und weltweiten Fällen insgesamt 27 Fälle von Amokfahrten gefunden.

\subsection{Datenlage zur Gesamtzahl von Amokfahrten in allen drei untersuchten Dekaden (1981 -1990, 1991 - 2000, 2001 - 2010)}

Die Datenlage_zu Amokfahrten der Dekade I (1980 - 1989) ist ein Ergebnis der Dissertationsarbeit von Schünemann (1991). Dieser wertete im Rahmen seiner Arbeit über den genannten 10-Jahreszeitraum mittels der kontentanalytischen Methode deutsche Pressebe- 
richte hinsichtlich eines möglichen Vorkommens von Amokfällen aus und fand 196 Fälle, die den wissenschaftlichen Amokkriterien entsprachen. Von den 196 Fällen, die er als echte Amoktaten identifizierte, hatten die Amoktäter in 98 Fällen Schusswaffen verwendet und in 48 Fällen atypische Waffen wie Pkws, Lkws oder Panzer. Somit waren in der Untersuchung von Schünemann 24,5 \% der Amokfälle Amokfahrten. Die Ergebnisse der Dekade II (1991 - 2000) wurden im Rahmen einer Publikation von Adler et al. (2006) mit denen der Dekade I verglichen. Nach Angaben von Frau D. Marx, die an dieser Studie mitwirkte, fanden sich in Dekade II nur zwei Fälle von Amokfahrten (persönliche Mitteilung am Telefon). In dieser Dissertationsarbeit erfolgte die Untersuchung der Häufigkeit des Vorkommens von Amokfahrten der Dekade_III (2001 - 2010). Unter Anwendung derselben kontentanalytischen Untersuchungsmethode, mit der bereits für die Dekaden I und II Presseberichte hinsichtlich des Vorkommens von Amok ausgewertet wurden, wurden über einen 10-Jahreszeitraum hinweg für das Gebiet der gesamten Bundesrepublik Deutschland 23 Fälle von Amokfahrten gefunden, die den wissenschaftlichen Amokkriterien entsprachen. Hinzu kamen 14 Amokfahrten, die sich im europäischen Ausland ereignet hatten und 10 Amokfahrten aus dem außereuropäischen Raum. Damit konnten insgesamt 47 Amokfahrten im untersuchten Zeitraum verifiziert werden. Die Gesamtzahl der Amokfahrten, die über einen Drei-Dekaden-Zeitraum in deutschen Presseartikeln gefunden wurden, beträgt damit 77. Setzt man die 23 Fälle deutscher Amokfahrten der dritten Dekade (durchschnittlich 2,3 Fälle pro Jahr) mit den jährlich etwa 300.000 schweren Verkehrsunfällen mit Personenschäden auf bundesdeutschen Straßen ins Verhältnis, so wird deutlich, dass Amokfahrten nur für einen verschwindend kleinen Anteil von 0,0008 \% dieser Unfälle verantwortlich sind. Schon dadurch bekommt man einen ersten Eindruck von der Seltenheit des Auftretens echter Amokfahrten.

\subsection{Definition Amokfahrten}

Um die Fälle echter Amokfahrten dieser Untersuchung untereinander vergleichen zu können und ebenso mit den Amokfahrten der vorangegangenen beiden Dekaden, braucht es operationalisierter Kriterien und einer Definition, was Amokfahrten sind und was nicht. Allgemein fällt Amok unter die Gewaltdefinition von Böker und Häfner (1973). Nach Adler (2001) hat aber Amok (wie auch die Amokfahrt als seine Sonderform) als homizidal- 
suizidale Gewalttat noch weitere typische Tatmerkmale: 1) Eine Amoktat liegt dann vor, wenn es durch die von einem Amoktäter ausgeübte Gewalt zum Tod mindestens eines Menschen gekommen ist bzw. wenn es dazu gekommen wäre, wenn nicht äußere, nicht in der Person des Täters liegende Gründe den Taterfolg verhindert hätten. 2) Weiterhin ist für eine Amoktat kennzeichnend, dass hier im Gegensatz zu anderen Gewalttaten des homizidalen Spektrums die ansonsten typische „ein Täter-ein Opfer-Konstellation“ verlassen und die Tat auf weitere zufällige Opfer ausgeweitet wird. 3) Kennzeichnend für eine Amoktat ist weiterhin, dass die Tat absichtsvoll und gerichtet ausgeführt wird und neben der primären homizidalen Tatrichtung immer auch einen suizidalen Aspekt enthält. Letzterer zeigt sich darin, dass ein Amoktäter die Tat ohne Rücksicht auf das eigene Leben ausführt und die meisten Amoktaten mit dem Suizid des Täters enden. In einigen Fällen stirbt der Amoktäter durch Fremdeinwirkung (z. B. Polizei). 4) Ein weiteres typisches Kenn-zeichen einer Amoktat ist ihr raptusartiger Beginn: Eine Amoktat wird vom Amokläufer oder Amokfahrer in einer Art „Zustand von Raserei“ begonnen und endet entweder durch Selbsttötung, Fremdtötung durch Sicherheitskräfte oder Überwältigung durch die Polizei. 5) Ein letztes wichtiges Merkmal einer Amoktat ist, dass ihr keinerlei politische, ethnische, religiöse oder kriminelle Tatmotive zugrunde liegen.

\subsection{Einschluss- und Ausschlusskriterien bei Amokfahrten}

Die Amokfahrt als eine Sonderform des Amoklaufs muss alle unter Kap. 2.7 aufgeführten Amokkriterien aufweisen. Hinzu kommen noch weitere Besonderheiten, die Amokfahrten von anderen schweren Verkehrsdelikten unterscheiden: Amokfahrer zeigen am Beginn ihrer Amokfahrt typischerweise ein impulsiv-raptusartiges Tatverhalten, das dem des aggressiven Autofahrers auf den ersten Blick ähnelt. Allerdings ist nur beim Amokfahrer eine Intentionalität oder Gerichtetheit des Tathandelns entsprechend der Kriterien der Menninger-Trias (Menninger 1938) gegeben.

Nicht wenige Amokfahrer könnten bei oberflächlicher Betrachtung der Gruppe der psychisch kranken Autofahrer zugeordnet werden, da psychische Erkrankungen bei Amokfahrern überdurchschnittlich häufig vorliegen. Auch hier ist es das Merkmal der Intentionalität, das den Amokfahrer vom psychisch kranken Unfallverursacher unterscheidet. Das Vorliegen einer akuten psychischen Erkrankung, eines krisenhaften akut-suizidalen $\mathrm{Zu}-$ 
stands oder einer akuten Alkohol- oder Drogenintoxikation führen bei einem Autofahrer stets zu einer deutlichen Minderung seiner Fahrsicherheit und einem erheblich gesteigerten Unfallrisiko. Ein Amokfahrer, nicht selten psychisch krank und gelegentlich zusätzlich intoxikiert, benutzt sein Auto als Tatwaffe in der Absicht, Menschen zu verletzen, zu töten und schwere Sachschäden anzurichten. Das Tatmotiv des Amokfahrers ist häufig äußerlich nicht erkennbar, weil es in vielen Fällen dem inneren Wahnerleben des Täters entspringt.

Amokfahrern und Suizidfahrern ist gemeinsam, dass beide ihr Auto intentional und gerichtet als Tatwaffe einsetzen. Während jedoch der Suizidfahrer beabsichtigt, ausschließlich sich selbst durch ein selbst inszeniertes Unfallereignis im Straßenverkehr zu töten, ist der Amokfahrer intentional darauf ausgerichtet, mit seinem Auto primär Gewalt gegen andere Verkehrsteilnehmer auszuüben, wobei er den eigenen Tod am Ende der Amokfahrt sucht oder billigend in Kauf nimmt.

Häufig werden in den Medien die Begriffe Geisterfahrer/Falschfahrer und Amokfahrer unrichtig synonym verwendet, obwohl es grundlegende Unterschiede zwischen einem Geisterfahrer bzw. Falschfahrer und einem echten Amokfahrer gibt: Geisterfahrer bzw. Falschfahrer sind aus unterschiedlichen, bereits wiederholt genannten Gründen im öffentlichen Straßenverkehr entgegen der Fahrtrichtung im Straßenverkehr unterwegs und stellen dadurch eine erhebliche Gefahr für entgegenkommende Autofahrer dar. Auch Amokfahrer sind häufig entgegen der Fahrtrichtung im Straßenverkehr unterwegs, wobei auch hier der entscheidende Unterschied die Intentionalität der Handlung ist: Während der Geisterfahrer durch fehlende Übersicht und/oder falsche Entscheidungen ohne es zu beabsichtigen, eine erhebliche Gefahr für andere Verkehrsteilnehmer darstellt, handelt der Amokfahrer, wenn er dem fließenden Verkehr entgegenfährt, absichtsvoll. Er will mit seiner Fahrt entgegen der Fahrtrichtung bewusst und absichtlich einen Frontalzusammenstoß mit einem anderen Fahrzeug herbeiführen in der Absicht, größtmöglichen Schaden anzurichten und das Leben anderer Menschen und sein eigenes auf spektakuläre Weise auszulöschen.

\subsection{Kontentanalytische Methoden}

Der Begriff Kontentanalyse (engl. content analysis) hat in der wissenschaftlichen Literatur die Bedeutung einer Text- oder Aussagenanalyse. Die Kontentanalyse stellt eine empiri- 
sche Forschungsmethode dar, mit der der Inhalt von Texten aller Art - so auch von Presseartikeln - quantitativ und qualitativ untersucht werden kann. Dabei wird der jeweilige Text in inhaltlich sinnvolle Texteinheiten (Items) zerlegt, die in der Folge codiert und danach statistisch ausgewertet werden. Mayring (2003) entwickelte dafür ein allgemeines inhaltsanalytisches Ablaufmodell, bei dem nach Festlegung von Art und Umfang des zu untersuchenden Materials als erstes eine Arbeitshypothese und die wissenschaftliche Fragestellung der Untersuchung festzulegen ist. Danach wird das Textmaterial für die weitere Auswertung in Text- oder Kodier-Einheiten, in der Folge Items genannt, zerlegt, die im weiteren Ablauf statistisch ausgewertet werden. So können dann am Ende des Untersuchungsprozesses Aussagen getroffen werden zur Fragestellung der Untersuchung und die Arbeitshypothese verifiziert oder falsifiziert werden.

In der wissenschaftlichen Literatur wird die kontentanalytische Methode zur Gewinnung wissenschaftlich fundierter Aussagen aus Presseartikeln nach wie vor angewandt: So untersuchten Calloway et al. (2006) mit Hilfe der kontentanalytischen Methode 25 Zeitungsartikel, die Informationen über die Verhütung einer Erkrankung an Cervix-Karzinom durch rechtzeitige Impfung von Frauen und Mädchen gegen HPV enthielten. Um diese Artikel zu gewinnen, wurden 285 US-amerikanische Zeitschriften der Jahre 2003 - 2005 ausgewertet. Die Fragestellung lautete, ob der Zusammenhang zwischen der Impfung und der Verhütung von Cervix-Karzinom in den Artikeln allgemeinverständlich dargelegt wurde. Im Ergebnis konnte gezeigt werden, dass die Hersteller des HPV-Impfstoffs in den untersuchten Artikeln den Zusammenhang zwischen PAP-Test, HPV-Impfung und Schutz vor CervixKarzinom nicht ausreichend deutlich und allgemeinverständlich dargestellt hatten, worauf diese nun im Text auf der Impfstoffverpackung diesen Zusammenhang wesentlich eindeutiger und verständlicher darstellten. Al-Bawab et al. (2018) werteten mit der kontentanalytischen Methode 220 Artikel über wissenschaftliche und Gesundheitsthemen, die im Zeitraum 2006 - 2015 in den auflagenstärksten britischen und US-amerikanischen Zeitungen erschienen waren unter der Fragestellung aus, ob es bei den Lesern eine Veränderung gegeben hat am Interesse an diesen Themen. Die Autoren kamen zu dem Ergebnis, dass es, was die Publikation von Artikeln zu Gesundheits- und wissenschaftlichen Themen betrifft, in den untersuchten Zeitschriften und im definierten Untersuchungszeitraum zu einem exponentiellen Anstieg kam. Daraus wurde geschlossen, dass das Interesse der Leser an diesen Themen stark zugenommen hat und sie auch oder gerade deswegen die untersuchten Zeitschriften kauften. 
In der hier vorliegenden eigenen Untersuchung wurde in 10 Jahrgängen dreier Thüringer Tageszeitungen der Jahre 2001 - 2010 nach Presseartikeln gesucht, die über Amokfahrten bzw. schwere Verkehrsunfälle berichteten, die wahrscheinlich durch eine Amokfahrt verursacht worden waren. Es wurden 76 Presseartikel gefunden, in denen sich Hinweise auf mögliche Amokfahrten fanden. Entsprechend des allgemeinen inhaltsanalytischen Ablaufmodells von Mayring (2003) wurde als erstes eine Arbeitshypothese und Fragestellungen gebildet (Kap. 1.7). Als erstes wurden diese 76 Artikel über 72 mögliche Amokfahrten (über einen Fall wurde fortlaufend in insgesamt vier Artikeln berichtet) anhand der wissenschaftlichen Amokkriterien (Kap. 2.7) überprüft. Bereits hier zeigte sich, dass von den gefundenen 72 möglichen Amokfahrten anhand der Datenlage der Artikel nur zwei als tatsächliche Amokfahrten verifiziert werden konnten. Da diese Anzahl viel zu klein war, um damit statistische Berechnungen durchzuführen, wurden in den in Kap. 2.3 genannten überregionalen Zeitungen und Zeitschriften desselben Untersuchungszeitraums nach weiteren Presseartikeln über Amokfahrten gesucht (optimaler Weise vom Zeitpunkt der Tat bis zur Urteilsverkündung), die Kriterien einer echten Amokfahrt erfüllten. Die beiden Thüringer Fälle gingen in die Anzahl der gesamtdeutschen Fälle echter Amokfahrten mit ein, wodurch eine Gesamtzahl von 23 Fällen zustande kam. Für die weitere Auswertung wurde der Inhalt der Presseberichte mit den echten Amokfahrten in 72 Items zerlegt (Kap. 2.9, Anhang Kap. 6.1), um auf diese Weise so viele Informationen wie nur irgend möglich zu gewinnen und anschließend zu verschlüsseln. Von den 72 Items wurde eine Übersicht in Tabellenform erstellt, wobei die x-Achse am oberen Rand die Fall-Nummern 1 - 23 abbildete und die y-Achse am linken Seitenrand die 72 Items (fortlaufend von oben nach unten). Den Inhalt der Tabelle bildeten die Angaben aus dem Presseartikel zum jeweiligen Fall in Form einer Zahlencodierung. Das so gewonnene Zahlenmaterial bildete die Grundlage für die weitere statistische Berechnung, die freundlicherweise von Herrn Dipl. Psych. H. Apel, Göttingen, durchgeführt wurde. Gerechnet wurde mit dem Auswertungsprogramm SPSS 10.0.7.x.

\subsection{Statistische Methoden}

Es wurde aus allen in der untersuchten Dekade ermittelten und diskontinuierlich auftretenden Amokfahrten ein Mittelwert gebildet (Kap. 3.2, Tab. 2). Eine weitere Mittelwert- 
bildung erfolgte hinsichtlich der Altersverteilung der Amokfahrer (Kap. 3.2, Tab. 3). Des Weiteren wurden Häufigkeitsverteilungen verwendet. Die mit dem genannten Auswertungsprogramm erstellten Häufigkeitstabellen wurden im Text graphisch dargestellt, um das Verteilungsverhalten der erhobenen Daten zu visualisieren. Es kamen dabei vor allem Säulendiagramme zur Anwendung (Kap. 3.2, Abb. 1 - 29). In dieser Untersuchung war die Altersverteilung der Amokfahrer zwischen 19 und 79 Jahren breit gestreut mit einer relativen Häufung zwischen 20 und 37 Jahren (Kap. 3.2, Abb. 2). 


\section{Ergebnisse}

\subsection{Amokfahrten im Spektrum anderer schwerer Verkehrsdelikte im Freistaat Thüringen 2001 - 2010}

Die Statistischen Jahrbücher für Thüringen der Jahre 2002 - 2011 (Thüringer Landesamt für Statistik 2002 - 2011) verzeichnen für das Gebiet des Freistaates Thüringen mit einem leichten Abwärtstrend eine durchschnittliche Einwohnerzahl von 2,3 Millionen Menschen. Auf diesem abgegrenzten Gebiet ereigneten sich nach Angabe der Statistischen Jahrbücher für Thüringen im untersuchten 10-Jahreszeitraum 88.142 schwere Verkehrsunfälle mit Personenschäden. Die jährlichen Unfallzahlen waren dabei nicht gleichbleibend hoch, sondern zeigten einen kontinuierlichen leichten Abwärtstrend.

Tabelle 2: Schwere Verkehrsunfälle mit Personenschäden in Thüringen 2001-2010

\begin{tabular}{|l|l|l|l|l|l|l|l|l|l|l|}
\hline Jahr & 2001 & 2002 & 2003 & 2004 & 2005 & 2006 & 2007 & 2008 & 2009 & 2010 \\
\hline Unfälle & 10.880 & 9.940 & 9.860 & 9.300 & 8.890 & 8.530 & 8.580 & 7.890 & 7.450 & 6.760 \\
\hline $\begin{array}{l}\text { Amok- } \\
\text { fahrten }\end{array}$ & 0 & 0 & 0 & 0 & 1 & 0 & 0 & 0 & 1 & 0 \\
\hline
\end{tabular}

Im Jahr 2001 wurden im Freistaat Thüringen 10.882 schwere Verkehrsunfälle mit Personenschäden statistisch erfasst. Die Grundannahme, die die Richtung des weiteren Vorgehens bei dieser Arbeit bestimmte, war, dass die lokale Presse über schwere Verkehrsunfälle mit Personenschäden, die sich in der jeweiligen Gegend ereignet hatten, sehr wahrscheinlich berichtet haben musste. Des Weiteren wurde von der Grundannahme ausgegangen, dass eine bisher unbekannte Anzahl dieser schweren Verkehrsunfälle durch eine Amokfahrt verursacht worden waren. Mit Hilfe der in Kap. 2.2 genannten Schlagwörter wurden mittels Online-Recherche im Zentralarchiv des Zeitungsverlags der drei Thüringer Tageszeitungen 10 Jahrgänge aller drei Thüringer Tageszeitungen durchsucht. Die Ergebnisse waren folgende: Der Jahrgang 2001 enthielt 12 Artikel, die Hinweise auf eine mögliche Amokfahrt enthielten. Von diesen Artikeln wurden 10 in der Thüringer Allgemeinen Zeitung (TA) gefunden, ein Artikel stammte aus der Ostthüringer Zeitung (OTZ) und ein weiterer aus der Thüringer Landeszeitung (TLZ). Bei 4 der 12 Artikel stand das Wort Amokfahrt in der Überschrift. Alle Zeitungsartikel dieses Jahrgangs, in denen es Hinweise für ein 
mögliches Vorliegen einer Amokfahrt gab, wurden mit Hilfe der Amokkriterien genauer überprüft. Im Ergebnis zeigte sich, dass der Inhalt von keinem der vier mit „Amokfahrt“ betitelten Zeitungsartikel einer Prüfung mit Hilfe der Amokkriterien standhielt - es sich also in keinem der Fälle um eine echte Amokfahrt gehandelt hat. Bei einem weiteren Artikel, bei dem in der Überschrift das Wort Amokfahrer vorkam, konnte nach derselben Überprüfung das Vorliegen einer echten Amokfahrt nicht ausgeschlossen, aber auch nicht verifiziert werden, die Datenlage zu gering war. Die 12 untersuchten Zeitungsartikel des Jahrgangs 2001 berichteten über 14 Fälle hochgefährlichen Fahrverhaltens im Straßenverkehr (davon ein Artikel mit drei Fällen), von denen vier vom Verfasser des Artikels als Amokfahrt bezeichnet worden waren. Die Artikel zu den 1fd. Nrn. der folgenden Absätze finden sich in Kap. 6.1. Tatsächlich handelte es sich in den untersuchten Zeitungsartikeln um: Aggressivität im Straßenverkehr (Kap. 1.1), $\mathrm{n}=4$ (lfd. Nrn. 4, 8, 9, 10), psychisch auffällige Autofahrer (Kap. 1.2), $\mathrm{n}=4$ (lfd. Nrn. 1, 2, 3, 7), Verdeckung einer Straftat: $\mathrm{n}=5$ (lfd. Nrn. 5/1, 5/2, 5/3, 13, 14) und einen Geisterfahrer (Kap. 1.4): n= 1 (lfd. Nr. 6).

Im darauffolgenden Jahr 2002 wurden in Thüringen 9.949 schwere Verkehrsunfälle mit Personenschäden statistisch erfasst. Unter den schon genannten Schlagworten fanden sich bei der Online-Recherche in drei Thüringer Lokalzeitungen sieben Artikel mit Meldungen über schwere Verkehrsunfälle. Davon waren fünf Artikel in der TA erschienen sowie je ein Artikel in den Tageszeitungen OTZ und TLZ. Fünf der sieben Artikel trugen die Überschrift Amokfahrt bzw. Amokfahrer. Nach Prüfung jedes einzelnen Falles anhand der Amokkriterien handelte es sich jedoch in keinem der Fälle um echte Amokfahrten, sondern um andere schwere Verkehrsstraftaten, verursacht durch: Aggressivität im Straßenverkehr (Kap. 1.1): $\mathrm{n}=1$ (lfd. Nr. 15), psychisch auffällige Autofahrer (Kap. 1.2): n = 2 (lfd. Nrn. 18, 19), Verdeckung einer Straftat: $\mathrm{n}=3$ (lfd. Nrn. 13, 14, 16) und einen Geisterfahrer (Kap. 1.4): $\mathrm{n}=1$ (lfd. Nr. 17).

Im nächsten Jahr, 2003, wurden durch das Thüringer Amt für Statistik 9.867 schwere Verkehrsunfälle mit Personenschäden erfasst. In der Thüringer Regionalpresse des Jahrgangs 2003 wurden auf dieselbe Weise wie schon zuvor beschrieben 12 Artikel über schwere Verkehrsunfälle oder erheblich verkehrsgefährdendes Verhalten von Autofahrern ausgewählt, bei denen es Hinweise auf ein mögliches Vorliegen einer Amokfahrt gab. Acht der Artikel entstammten der TA, je zwei der TLZ bzw. der OTZ. Bei zweien der 12 Artikel stand das Wort Amokfahrt bzw. Amokfahrer in der Überschrift. Aber auch in diesen Fällen 
gab es keine Übereinstimmung zwischen der Ankündigung in der Überschrift und dem tatsächlichen Sachverhalt im Artikel: Während es sich bei den zwei Artikeln, die in der Überschrift ankündigten, von einer Amokfahrt zu berichten, gerade nicht um tatsächliche Amokfahrten handelte, sprach in zwei weiteren Artikeln (die jedoch das Wort Amokfahrt bzw. Amokfahrer nicht in der Überschrift verwendeten) Einiges dafür, dass es sich es sich in beiden Fällen um echte Amokfahrten gehandelt haben könnte. Leider war die Datenlage in beiden Artikeln nicht umfangreich und aussagekräftig genug, sodass beide nicht als gesicherte Amokfahrten gewertet werden konnten. In den anderen Fällen handelte es sich um: Aggressivität im Straßenverkehr (Kap. 1.1): $\mathrm{n}=2$ (lfd. Nr. 20, psychisch auffällige Autofahrer (Kap. 1.2): $\mathrm{n}=2$ (lfd. Nrn. 22, 23), jugendlichen Leichtsinn: $\mathrm{n}=3$ (lfd. Nrn. 21, 28, 30), Kontrolle über das Fahrzeug verloren: $\mathrm{n}=2$ (1fd. Nrn. 26, 31), einen Geisterfahrer (Kap. 1.4): $\mathrm{n}=1$ (lfd. Nr. 29) und Amokfahrt (Kap. 1.5): $\mathrm{n}=2$, eine davon nicht gesichert (1fd. Nrn. 24, 27).

Das statistische Jahrbuch Thüringen 2004 verzeichnet 9.306 schwere Verkehrsunfälle mit Personenschäden. Unter den schon genannten Schlagworten fanden sich in den 3 Regionalzeitungen des Jahrgangs 2004 fanden sich lediglich vier Artikel über schwere Verkehrsunfälle, von denen drei das Wort Amokfahrt in der Überschrift führten. Von diesen vier Artikeln stammten drei aus der TLZ und einer aus der TA. Bei zwei der vier Artikel stellte sich bei genauer Überprüfung heraus, dass der Begriff Amokfahrt in der Überschrift nicht den Sachverhalt beschrieb, von dem im Artikel die Rede war. Bei den beiden anderen Artikeln, von denen einer mit Amokfahrt betitelt war und der andere nicht, sprach Einiges für ein mögliches Vorliegen einer echten Amokfahrt: In einem dieser beiden Fälle (Nr. 32) lässt das geschilderte Verhalten des Autofahrers darauf schließen, dass er sein Auto als Waffe einsetzte, um damit zielgerichtet a) andere Verkehrsteilnehmer zu gefährden und ihnen Schaden zuzufügen und b) am Ende seinem Leben ein Ende zu setzen. Konkret verursachte er gezielt und absichtsvoll Auffahrunfälle bei mehreren anderen Fahrzeugend kurz hintereinander, bis er zuletzt frontal auf einen LKW auffuhr und dabei den Tod fand. Im zweiten Fall (Nr. 34) wendete der Unfallverursacher plötzlich und ohne erkennbaren Grund auf der Autobahn, um danach entgegen der Fahrtrichtung mit hoher Geschwindigkeit frontal und auf einen entgegenkommenden Pkw aufzufahren. Dabei fand der Unfallverursacher den Tod, sein Unfallgegner wurde schwer verletzt, es entstand Totalschaden an beiden Fahrzeugen. Obwohl es in beiden Fällen deutliche Hinweise für ein mögliches Vorliegen einer Amokfahrt gibt, war leider bei beiden Artikeln die Datenlage zu gering, um die 
Amokfahrten sicher verifizieren zu können. Es handelte sich bei den Verkehrsstraftaten um: Verdeckung einer anderen Straftat: $\mathrm{n}=2$ (1fd. Nrn. 33, 35) und zwei mögliche Amokfahrten (Kap. 1.5): $\mathrm{n}=2$ (1fd. Nrn. 32, 34).

Im Jahr 2005 wurden auf dem Gebiet des Freistaats Thüringen 8.893 schwere Verkehrsunfälle mit Personenschäden registriert. Es fanden sich aus diesem Zeitraum sechs Artikel, die über fünf Fälle möglicher Amokfahrten berichteten (über einen Fall gab es zwei Artikel). Obwohl keiner der Artikel mit der Überschrift Amokfahrt betitelt war, befand sich darunter erstmals_ein gesicherter Fall einer echten Amokfahrt (lfd. Nr. 37). In den anderen Artikeln handelte es sich in Wirklichkeit um: Fahrfehler hochbetagter Autofahrer: $\mathrm{n}=2$ (lfd. Nrn. 40, 42), einen psychisch auffälligen Autofahrer (Kap. 1.2): n = 1 (lfd. Nr. 36), 3), einen Geisterfahrer (Kap. 1.4): $\mathrm{n}=1$ (lfd. Nr. 39) und zwei Amokfahrten (Kap. 1.5): $\mathrm{n}=2$ (lfd. Nrn. 37, 41, eine davon gesichert).

Da über den Fall Nr. 37 in der Lokalpresse mehrere Artikel mit z.T. umfangreicher Berichterstattung erschienen waren, standen hier deutlich mehr Informationen zur Verfügung als bei den sonst eher kurz gehaltenen Artikeln über schwere Verkehrsunfälle und besonders verkehrsgefährdendes Verhalten von motorisierten Verkehrsteilnehmern. Da dieser Fall geradezu exemplarisch alle typischen Merkmale einer echten Amokfahrt aufweist, wird er als Fallvignette $1 \mathrm{im}$ Kapitel 3.1 ausführlich dargestellt. Auch Fall Nr. 41 weist Merkmale einer echten Amokfahrt auf. Allerdings gab es keine Informationen über den Amokfahrer. Aus der Zeugenschilderung wissen wir lediglich, dass eine Person bei Dunkelheit auf der Autobahn entgegen der Fahrtrichtung mit seinem unbeleuchteten Fahrzeug mit hoher Geschwindigkeit direkt auf das Fahrzeug des Tatzeugen zufuhr. Dieser bemerkte das unbeleuchtete Fahrzeug, das auf ihn zuraste, erst im allerletzten Moment vor dem ansonsten unvermeidlichen Frontal-Crash beider Fahrzeuge. Er blieb unverletzt, weil es ihm ganz zuletzt noch gelang, sein Fahrzeug nach ganz rechts zu lenken und gerade noch rechtzeitig die Fahrbahn zu verlassen. Da der Amokfahrer seine Fahrt nicht unterbrach, sondern weiter unbeleuchtet entgegen der Fahrtrichtung davonraste, gibt es keine Informationen über seine Person und seine Tatmotive.

Im Jahr 2006 ereigneten sich auf Thüringens Straßen 8.539 schwere Verkehrsunfälle mit Personenschäden, die statistisch erfasst wurden. In der lokalen Presse fanden sich neun Artikel über schwere Verkehrsunfälle und verkehrsgefährdendes Verhalten, davon sechs Artikel in der TA, zwei Artikel in der OTZ und ein Artikel in der TLZ. Zwei der neun Arti- 
kel trugen die Überschrift Amokfahrt, Sieben der neun Artikel berichteten im Text über Fälle möglicher Amokfahrten. Zwei der Artikel befassten sich mit dem weiteren Fortgang nach dem Ende der Amokfahrt vom 01.04.2005 (lfd. Nrn. 37, 46, 47) Bei den schweren Verkehrsereignissen der anderen Artikel handelte es sich in Wirklichkeit um Verkehrsstraftaten, verursacht durch erheblich alkoholisierte Autofahrer: $n=2$ (lfd. Nrn. 48, 50), Verdeckung einer anderen Straftat: $n=1$ (lfd. Nr. 43) und 3) Geisterfahrer (Kap. 1.4): $n=4$ (1fd. Nrn. 42, 44, 45, 49).

Im Jahr 2007 ereigneten sich auf den Thüringer Straßen 8.588 statistisch erfasste schwere Verkehrsunfälle mit Personenschäden. Unter den bekannten Schlagworten fanden sich im gesamten Jahrgang aller drei Thüringer Lokalzeitungen nur drei Berichte über schwere Verkehrsunfälle bzw. gefährliches Fahrverhalten im Straßenverkehr - zwei davon in der OTZ und Artikel in der TA. Bei den Verkehrsstraftaten, über die in den Artikeln berichtet wurde, handelte es sich jedoch in keinem der Fälle um eine Amokfahrt, sondern um: Verdeckung einer anderen Straftat: $n=1$ (lfd. Nr. 51) und Geisterfahrer (Kap.1.4): $n=2$ (lfd. Nrn. 52, 53).

Im Jahr 2008 nahm die Zahl schwerer Verkehrsunfälle mit Personenschäden weiter ab, sodass nur noch 7.893 von ihnen Eingang in das Statistische Jahrbuch Thüringen fanden. In den Thüringer Lokalzeitungen des Jahrgangs 2008 fand die Untersucherin mit Hilfe der genannten Suchbegriffe vier Artikel über mögliche Amokfahrten, drei davon in der TA und einen Artikel in der OTZ. In keinem der Artikel fanden sich Hinweise auf eine tatsächliche Amokfahrt. Es handelte sich hier in Wirklichkeit um erheblich verkehrsgefährdendes Verhalten, verursacht durch einen psychisch auffälligen Autofahrer (Kap. 1.2): $\mathrm{n}=1$ (lfd. Nr. 56), Geisterfahrer (Kap. 1.4): $\mathrm{n}=2$ (lfd. Nrn. 55, 57) und ein akutes gesundheitliches Ereignis: $\mathrm{n}=1$ (1fd. Nr. 58).

Im Jahr 2009 ereigneten sich in Thüringen 7.456 schwere Verkehrsunfälle mit Personenschäden. In den lokalen Zeitungen des Jahrgangs 2009 wurden mit Hilfe genannten Schlagworte 11 Artikel über schwere Unfälle oder Verkehrsstraftaten gefunden, von denen zwei das Wort Amokfahrt in der Überschrift trugen. 7 der 11 Artikel waren in der TA erschienen, drei in der OTZ und ein Artikel in der TLZ. In einem Fall handelte es sich tatsächlich um eine Amokfahrt, in den übrigen 10 Artikeln handelte es sich tatsächlich um fahrlässiges Fehlverhalten: $\mathrm{n}=2$ (lfd. Nrn. 66, 67), psychisch auffällige Autofahrer (Kap.1.2): $\mathrm{n}=2$ (lfd. Nrn. 64, 68), Verdeckung einer anderen Straftat: $\mathrm{n}=2$ (lfd. Nrn. 61, 
63), grober Unfug: $n=1$ (lfd. Nr. 60), Geisterfahrer: (Kap.1.4): $n=3$ (lfd. Nrn. 59, 62, 65) und einen Amokfahrer (Kap.1.5): $\mathrm{n}=1$ (lfd. Nr. 54, Fallvignette 2).

Im letzten Jahr der untersuchten Dekade war die Zahl der schweren Verkehrsunfälle mit Personenschäden in Thüringen nochmals zurückgegangen, 6.769 von ihnen wurden statistisch erfasst. Im Jahr 2010 erbrachte die Zeitungsrecherche acht Artikel über schwere Verkehrsereignisse, wovon ein Artikel das Wort Amokfahrt in der Überschrift führte. Ein Artikel berichtete über zwei Fälle, sodass es neun Fälle bei acht Artikeln gab. Sieben Artikel stammten aus der TA, je ein Artikel aus der TLZ und aus der OTZ. Allerdings ging es in keinem der neun Artikel tatsächlich um eine Amokfahrt. Bei den Verursachern polizeilich erfasster Verkehrsstraftaten handelte es sich stattdessen um psychisch auffällige und/oder intoxikierte Autofahrer (Kap. 1.2): $\mathrm{n}=2$ (lfd. Nrn. 69, 74), einen Fall von Suizid mit dem Auto (Kap. 1.3): $\mathrm{n}=1$ (lfd. Nr. 75) und Geisterfahrer (Kap. 1.4): $\mathrm{n}=6$ (lfd. Nrn. 70/1, $70 / 2,71,72,73,76)$.

Zusammenfassend ist mit einiger Ernüchterung zu konstatieren, dass für den Untersuchungszeitraum 2001 - 2010 nur zwei Fälle echter Amokfahrten gesichert werden konnten. Im Vergleich dazu, dass sich in diesem Untersuchungszeitraum auf Thüringer Straßen 88.142 schwere Verkehrsunfälle mit Personenschäden ereigneten und nur durch zwei Amokfahrten (mehr oder weniger) schwere Verkehrsunfälle verursacht wurden, machen Amokfahrten rein rechnerisch einem Anteil von 0,0023 \% am Gesamtunfallgeschehen aus und stellen somit extrem seltene Verkehrsereignisse dar. Sechs weitere Fälle in dieser Untersuchung, die ebenfalls Merkmale einer Amokfahrt aufwiesen, konnten als tatsächliche Amokfahrten weder verifiziert noch falsifiziert werden, weil die Zeitungsartikel, die über sie berichteten, nicht genug Informationen hergaben, um damit eine vollständige Prüfung anhand der Amokkriterien durchführen zu können. Aber auch wenn es genügend Informationen über diese wahrscheinlichen Amokfahrten in den Presseartikeln gegeben hätte und wir dadurch auf acht statt zwei Amokfahrten im untersuchten 10-Jahreszeitraum kämen, wäre der Anteil der Amokfahrten am Unfallgeschehen auf den Thüringer Straßen mit $0,0091 \%$ immer noch außerordentlich gering.

Ebenfalls interessant ist die Verteilung der Häufigkeiten bei den anderen schweren Verkehrsereignissen, bei denen es sich - ungeachtet der journalistischen Bezeichnung in den untersuchten Artikeln der Thüringer Lokalpresse - nicht um echte Amokfahrten handelte: 
In sieben der 73 Thüringer Fälle $(9,6 \%)$ aus 76 Zeitungsartikeln waren es aggressive Autofahrer („Raser“, Kap. 1.1), die schwere Verkehrsunfälle mit Personenschäden verursachten. Bei 16 von 73 Fällen (21,1\%) waren die Unfallverursacher psychisch auffällige bzw. erheblich alkohol- oder Drogen intoxikierte Autofahrer (Kap. 1.2). Nur in einem von 73 Fällen $(1,3 \%)$ handelte es sich mit großer Wahrscheinlichkeit um Suizid mit dem Auto (Kap. 1.3). Geisterfahrer/Falschfahrer (Kap. 1.4) stellten mit 17 von 73 Fällen einen Anteil von 23,3 \% bei den Unfallverursachern. Den größten Anteil aber mit 19 von 73 Fällen (26 \%) machten Straftäter aus, die, mit dem Auto auf der Flucht vor der Polizei, durch Fahren mit hoher Geschwindigkeit und durch riskante Fahrmanöver ihrer Verhaftung entkommen wollen und wegen ihrer rücksichtslosen und unberechenbaren Fahrweise eine erhebliche Gefahr für die anderen Verkehrsteilnehmer darstellen. Seltene Ereignisse, die bei den untersuchten Fällen ebenfalls vorkamen, waren fahrlässiges Fehlverhalten im Straßenverkehr $(2,73 \%)$, schwere Fahrfehler hochbetagter Autofahrer (2,73\%), aus unbekannter Ursache plötzlich die Kontrolle über das Fahrzeug verloren (2,73\%)), grober Unfug und jugendlicher Leichtsinn (je 1,3\%) und akutes gesundheitliches Ereignis $(n=1)$. Die seltenen Ereignisse machen insgesamt einen Anteil von ca. 12,3 \% von allen 73 Fällen der 76 untersuchten Presseberichte aus.

Weil ursprünglich geplant war, die Thüringer Fälle echter Amokfahrten kontentanalytisch auszuwerten (was sich dann aber aufgrund der geringen Fallzahlen als nicht durchführbar erwies), wurden die aus den Presseartikeln gewonnenen Informationen den definierten Items (im Anhang, Kap. 6.1) zugeordnet. Diese Informationen stellen sich wie folgt dar:

Bei sechs der acht Amokfahrten, die sich in Thüringen im Untersuchungszeitraum ereigneten (zwei gesicherte, sechs mit nicht ausreichender Datenlage) ist das Alter des Amokfahrers bekannt. Es stellte sich mit einer Altersspanne zwischen 19 und 57 Jahren als breit gestreut mit einem Altersdurchschnitt von 35,3 Jahren dar. Alle acht Amokfahrer waren männlich. Da es in keinem der Artikel Angaben zur ethnischen Herkunft des Amokfahrers gab, sich aber die Amokfahrten in einem Zeitraum ereigneten, als Menschen nichtdeutscher Abstammung in Thüringen als einem der neuen Bundesländer noch eine sehr kleine Minderheit darstellten, kann davon ausgegangen werden, dass alle acht Amokfahrer Deutsche waren. Auch zum Familienstand, Berufsausbildung und Beschäftigungsverhältnis der Thüringer Amokfahrer liegen keine Angaben vor. Von den beiden Tätern, deren Amokfahrten als gesichert gelten können (1fd. Nrn. 37, 54), wissen wir aus den Presseberichten, dass 
beide schon vor der Tat psychisch krank waren. Von beiden wird berichtet, dass bei ihnen eine paranoide Schizophrenie vorlag, bei dem Amokfahrer mit der lfd. Nr. 54 noch zusätzlich akute Suizidalität. Vom Amokfahrer mit der lfd. Nr. 37 berichtete die Presse in mehreren Artikeln, dass sich dieser im Vorfeld seiner Amokfahrt in stationär-psychiatrischer Behandlung befand, dort keine Behandlungseinsicht zeigte und die Klinik gegen ärztlichen Rat verließ.

\section{Fallvignette 1: Die Amokfahrt des Holger Z. (Ifd. Nr. 37)}

Über die Amokfahrt des damals 23jährigen Holger Z. aus Bad Salzungen schrieben nicht nur die Thüringer Lokalzeitungen, es wurde wegen der außergewöhnlichen Tatumstände bundesweit darüber berichtet: Am 29.03.2005 hatte der an einer schizophrenen Psychose erkrankte Holger Z. die Psychiatrische Klinik Bad Salzungen (Wartburgkreis) gegen ärztlichen Rat verlassen und war nach Hause zurückgekehrt. Am frühen Morgen des 01.04.2005 randalierte Holger Z. vor dem Nachbarhaus und versuchte, dort Feuer zu legen (was ihm nicht gelang). Wenig später hielt in seiner unmittelbaren Nähe ein Lieferwagen, wobei der Fahrer, in Eile, die Wagentür nicht und den Zündschlüssel stecken ließ. Holger Z. bemerkte dies, sprang in den Lieferwagen und raste mit dem gestohlenen Lkw davon. Mit hoher Geschwindigkeit fuhr er von Bad Salzungen über die Bundesstraße 19 südwärts durch den Wartburgkreis und weiter durch den daran angrenzenden Landkreis SchmalkaldenMeiningen. Auf seiner Amokfahrt richtete er erhebliche Schäden an, beispielsweise, indem er mit seinem Fahrzeug geschlossene Bahnschranken durchbrach und Zäune umfuhr. Mehrfach waren dabei auch Menschen in akuter Lebensgefahr, z. B. als der Täter in einer Ortsdurchfahrt in ein direkt an der Straße gelegenes Haus hineinfuhr und dabei im Wohnzimmer zum Stehen kam. Es gelang Holger Z. jedoch, sein Fahrzeug zurückzusetzen und weiterzufahren. Um den Amokfahrer zu stoppen, wurde ein Lkw-Fahrer von der Polizei gebeten, sein Fahrzeug quer über die B19 zu stellen - genau dort, wo erwartet wurde, dass Holger Z. demnächst vorbeifahren würde. Die Polizei hatte richtig vermutet, wenig später kam Holger $Z$ auf der B 19 mit dem Lieferwagen herangerast - direkt auf den quergestellten Lkw zu, aus dem der Fahrer gerade (auf der Holger Z. zugewandten Fahrerseite) mit dem Rücken zur Fahrbahn ausstieg. Holger Z. fuhr ungebremst und mit hoher Geschwindigkeit auf den Lkw-Fahrer zu und fügte ihm beim Aufprall tödliche Quetschungsverlet- 
zungen zu. Kurz darauf setzte Holger Z. sein Fahrzeug erneut zurück, um weiterzufahren. Da beidseits neben dem quergestellten Lkw Polizeiautos als Absperrung standen waren, fuhr er auf ein Polizeiauto auf und schob dieses in den Straßengraben. So konnte er an dem quergestellten Lkw vorbeifahren und raste weiter über die B 19. Der Amokfahrer kam erst dadurch zum Stehen, nachdem er auf eine neben der Straße gelegene Mauer aufgefahren war und sein Fahrzeug danach nicht mehr ansprang. Erst danach konnte ihn die Polizei überwältigen und festzunehmen.

Am 29.05.2006 fand am Landgericht Meiningen die Hauptverhandlung im Strafverfahren gegen den inzwischen 24jährigen Holger Z. statt. Von der Staatsanwaltschaft Meiningen wurde dieser wegen Mordes, Verkehrsgefährdung, Diebstahls, Sachbeschädigung und Bedrohung angeklagt. Der Tatvorwurf Mord wurde erhoben, weil die Staatsanwaltschaft aufgrund der polizeilichen Ermittlungsergebnisse davon überzeugt war, dass Holger Z. bei seiner Amokfahrt den Tod des Lastwagenfahrers mindestens billigend in Kauf nahm. Aus einer Pressemeldung über die Hauptverhandlung des Holger Z. geht hervor, dass dieser sich vor Gericht auffällig verhalten habe: Er habe die Verhandlung wiederholt durch Zwischenrufe gestört und auch ansonsten ein Verhalten gezeigt, dass die Staatsanwaltschaft in ihrer Auffassung bestärkte habe, dass der Angeklagte psychisch krank und deshalb sehr wahrscheinlich schuldunfähig ist. Daher forderte sie anstelle einer Strafe seine weitere Unterbringung in einem Psychiatrischen Krankenhaus. Am 31.05.2006 fand die Urteilsverkündung statt. Das Landgericht Meiningen folgte in seinem Urteil dem Antrag der Staatsanwaltschaft Meiningen und wies Holger Z. in eine geschlossene Psychiatrische Klinik ein. In der Urteilsbegründung hieß es, dass der inzwischen 24jährige seit 2004 wiederholt erheblich verhaltensauffällig in der Öffentlichkeit in Erscheinung getreten sei. Zum Zeitpunkt der Tat wie auch zwei Tage zuvor, als er gegen ärztlichen Rat die Psychiatrische Klinik Bad Salzungen verließ, habe bei ihm eine akute Psychose bestanden. In seinem Schlusswort vor Gericht sagte Holger Z.: „, Ich will eine Behandlung. Es ist mir wichtig, dass das, was geschehen ist, nicht wieder geschehen kann. “

Der Fall der Amokfahrt des Holger Z. enthält alle Amokkriterien: Es handelt sich um eine schwerwiegende Gewalttat, bei der eine andere Person durch den Amokfahrer getötet wurde (Homizid). Die typische „ein Täter-ein Opfer-Konstellation“ sonstiger homizidaler Taten wurde dadurch erweitert, dass der Amokfahrer in seinem Furor mit seinem Tatfahrzeug weitere erhebliche Sachschäden an anderen Pkws, Häusern, Zäunen und Verkehrsleit- 
einrichtungen anrichtete. Seine rücksichtslose Fahrweise war geeignet, dass noch weitere Personen zu verletzen oder gar zu töten. Die Tat verlief intentional, und darauf gerichtet, einen größtmöglichen Schaden anzurichten. Die Tatausführung erfolgte ohne Rücksicht auf das eigene Leben, den eigenen Tod billigend in Kauf nehmend. Die Tat begann Raptus artig-impulsiv am frühen Morgen - anfangs mit tätlicher Gewalt gegen die Bewohner des Nachbarhauses. Sie wurde zur Amokfahrt, als durch Unachtsamkeit eines Lieferanten ein unverschlossener fahrbereiter Lkw zufällig in unmittelbarer Nähe des Täters abgestellt wurde. Homizidale und suizidale Elemente erscheinen gleichzeitig und tateinheitlich, da das Fahrverhalten des Täters von einer Rücksichtslosigkeit geprägt war, die ihn leicht auch sein eigenes Leben hätte kosten können. Ein äußeres Tatmotiv ist nicht erkennbar, die Tat ist nur aus dem Wahnerleben des Täters zu erklären. Politische, ethnische, religiöse oder kriminelle Motive können in diesem Fall ausgeschlossen werden. Der Täter dieser Fallvignette gehört zur Gruppe der psychisch kranken Amokfahrer.

\section{Fallvignette 2: N.N., 23-jähriger, psychisch kranker Amokfahrer (Ifd. Nr. 54)}

Die Ostthüringer Zeitung vom 23.05.2009 brachte auf ihrer Regionalseite „Jena und Umgebung“ einen Artikel mit der Überschrift: „Es war wie ein Amoklauf“. In dem Artikel wurde über einen 23jährigen Mann berichtet, bei dem schon im Vorfeld eine paranoide Schizophrenie bekannt war. In suizidaler Absicht sei er am 22.05.2009 mit seinem Auto auf der Bundesstraße 7 in der Nähe von Jena entgegen der Fahrtrichtung unterwegs gewesen. Mit hoher Geschwindigkeit und nicht angeschnallt sei er direkt auf einen Kleinbus zugefahren, in dem außer der Fahrerin noch ihre drei, fünf, sieben, zwölf und fünfzehn Jahre alten Kinder saßen. Den fast unvermeidlichen Frontalzusammenstoß habe die Fahrerin des Kleinbusses in letzter Sekunde dadurch verhindern können, dass sie ihr Fahrzeug scharf rechts gelenkt und über eine Böschung auf eine Wiese hinuntergefahren sei. Dabei überschlug sich der Kleinbus und landete im Straßengraben. Wie durch ein Wunder erlitten die Fahrerin wie auch ihre vier Kinder nur leichte Verletzungen. Die Fahrerin gab später gegenüber der Polizei an, dass der der ihr entgegenkommende Autofahrer ihr Fahrzeug sehr wohl bemerkt habe, Gas gegeben und mit hoher Geschwindigkeit auf sie zugefahren sei. Weiter wurde in dem Artikel berichtet, dass der Amokfahrer wenig später von der Polizei gefasst wurde. Es wurde ein Strafverfahren gegen ihn eingeleitet und er wurde aufgrund 
seiner psychiatrischen Vorerkrankung forensisch-psychiatrisch begutachtet. Aufgrund des Ergebnisses des Gutachtens wurde seitens des Gerichts seine Schuldunfähigkeit zum Zeitpunkt der Tat festgestellt und seine Unterbringung in einem Psychiatrischen Krankenhaus angeordnet.

Auch diese Amokfahrt erfüllt alle Amokkriterien: Der psychisch kranke Amokfahrer war im öffentlichen Straßenverkehr unterwegs, um mit seinem Auto Suizid zu begehen. Wäre er gegen einen Baum, einen Betonpfeiler oder einen Lkw gefahren, wäre er tatsächlich als Suizidfahrer einzuordnen. Da er aber nicht angeschnallt und mit hoher Geschwindigkeit entgegen der Fahrtrichtung auf ein nicht wesentlich größeres Fahrzeug zuraste (was er im Näherkommen hätte sehen müssen), hat er zumindest billigend in Kauf genommen, dass bei einem zu erwartenden Frontalzusammenstoß nicht nur er selbst, sondern mindestens auch noch der Fahrer des entgegenkommenden Fahrzeugs getötet wird. Damit sind alle Amokkriterien erfüllt: Der Amokfahrer will durch einen Autounfall sterben (Suizidabsicht). Er nimmt es dabei billigend in Kauf, dass mindestens eine weitere ihm unbekannte Person bei dem von ihm angestrebten Frontalzusammenstoß mit einem anderen Fahrzeug stirbt (Homizid-Komponente der Tat). Auch dieser Täter gehört zur Gruppe der psychisch kranken Amokfahrer. Bei der sehr geringen Zahl echter Amokfahrten $(n=2)$, die im Zeitraum 2001 - 2010 auf dem Gebiet des Freistaates Thüringen aus Presseberichten lokaler Zeitungen sicher verifiziert werden konnten, sind diese beiden Amokfahrer nicht repräsentativ für die Gesamtheit aller Amokfahrer-Typen. Es könnte aber ein Hinweis darauf sein, dass möglicherweise junge männliche Autofahrer mit einer akuten psychischen Erkrankung in einer krisenhaften Lebenssituation in der Gesamtgruppe der Amokfahrer überrepräsentiert sind.

Von den 88.140 schweren Verkehrsunfällen mit Personenschäden, die im Zeitraum 2001 - 2010 auf dem Gebiet des Freistaates Thüringen polizeilich registriert wurden, konnte nur in zwei Fällen eine Amokfahrt als Unfallursache gesichert werden. In sechs weiteren Fällen sprachen einige Hinweise für ein Vorliegen einer Amokfahrt. Die Daten, die aus den Pressemitteilungen gewonnen werden konnten, reichten jedoch bei diesen Fällen nicht aus, um daran alle Amokkriterien prüfen zu können. Die geringe Anzahl von zwei sicheren Amokfahrten war nicht ausreichend, um eine kontentanalytische Auswertung durchführen zu können. 


\subsection{Amokfahrer in Deutschland 2001 - 2010}

Im Untersuchungszeitraum 2001 - 2010, der der dritten Dekade der Amok-Gesamtstudie entspricht, ereigneten sich in Deutschland insgesamt 3.351.408 schwere Verkehrsunfälle mit Personenschäden (Quelle: Statistische Jahrbücher der Bundesrepublik Deutschland 2001 - 2011). Die jährlichen Unfallzahlen variierten allerdings voneinander mit der Tendenz eines kontinuierlichen Rückgangs. Einschließlich der beiden Thüringer Fälle konnten für das Gebiet der gesamten Bundesrepublik im Untersuchungszeitraum 23 echte Amokfahrten verifiziert werden.

Tabelle 3: Erfasste Verkehrsunfälle mit Personenschäden in Deutschland

\begin{tabular}{|l|l|l|l|l|l|l|l|l|l|l|}
\hline A & 2001 & 2002 & 2003 & 2004 & 2005 & 2006 & 2007 & 2008 & 2009 & 2010 \\
\hline B & 375.345 & 362.054 & 354.534 & 339.310 & 336.619 & 327.984 & 335.845 & 320.614 & 310.806 & 288.297 \\
\hline C & 3 & 0 & 4 & 4 & 3 & 1 & 2 & 2 & 3 & 1 \\
\hline
\end{tabular}

$\mathrm{A}=$ Jahr $\mathrm{B}=$ Unfälle $\mathrm{C}=$ Amokfahrten

Für alle Abbildungen gilt: $\mathrm{n}$ (Gesamtzahl der Amokfahrer) $=23$

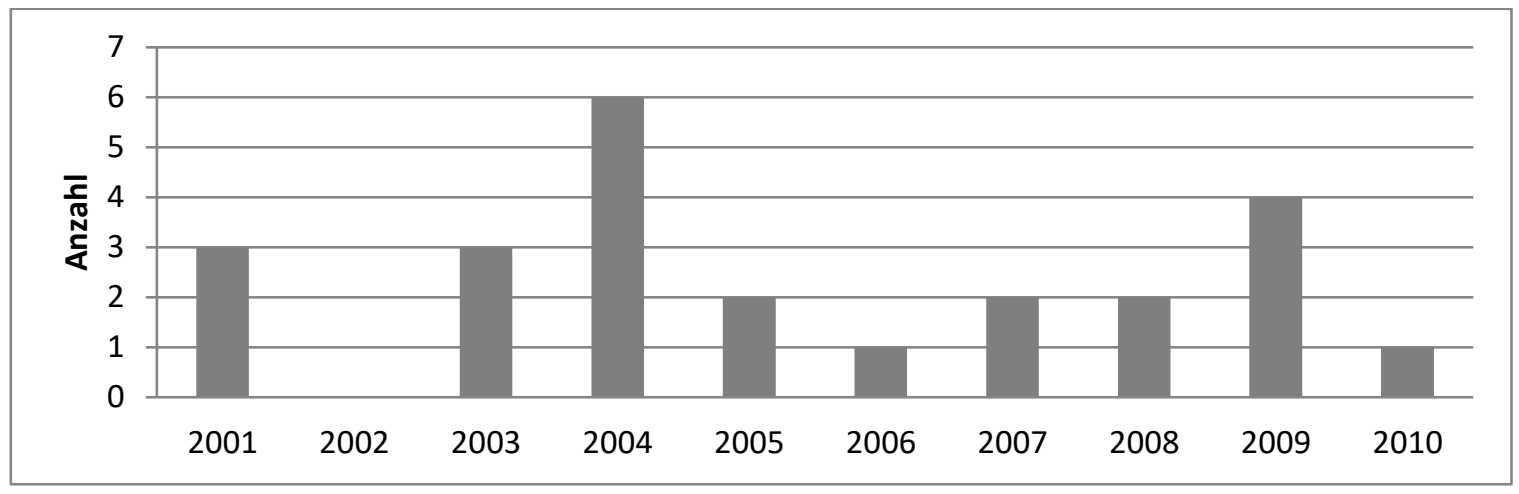

Abbildung 1: Darstellung der Häufigkeiten der Amokfahrten in Deutschland

Die Bundesrepublik Deutschland hatte in den Jahren 2001 - 2010 etwa 82 Millionen Einwohner. Dabei war ein leichter, aber kontinuierlicher Bevölkerungsrückgang von 550.000 Einwohnern innerhalb von zehn Jahren zu verzeichnen. Durchschnittlich kam also auf bundesdeutschen Straßen jährlich zu durchschnittlich 335.141 schweren Verkehrsunfällen mit Personenschäden, von denen anhand der Ergebnisse dieser Untersuchung nur in 2,3 Fällen eine Amokfahrt die Unfallursache darstellte. Somit betrug der Anteil von Amokfahr- 
ten am jährlichen Gesamtunfallgeschehen lediglich 0,007 \% und zeigt wie schon in Thüringen, dass Amokfahrten beim Gesamtunfallgeschehen nur eine sehr geringe Rolle spielen.

Das Täteralter streute in dieser Untersuchung ohne erkennbaren Altersgipfel zwischen 19 und 79 Jahren mit einem Altersdurchschnitt von 35,5 Jahren. Die Altersverteilung war in dieser Untersuchung bei einer relativ kleinen Gesamtgruppe von $n=23$ breit gestreut mit einer Altersgrenze zwischen 19 und 79 Jahren und zeigte kein Auftreten von Altershäufigkeiten.

Tabelle 4: Altersverteilung aller Amokfahrer in Deutschland

\begin{tabular}{|l|c|c|c|c|c|c|c|c|c|c|c|c|c|c|c|c|}
\hline Alter & 19 & 20 & 23 & 24 & 27 & 28 & 31 & 33 & 34 & 35 & 36 & 41 & 43 & 52 & 64 & 79 \\
\hline & & & & & & & & & & & & & & & & \\
\hline Anzahl & 2 & 1 & 2 & 2 & 2 & 1 & 2 & 1 & 2 & 1 & 2 & 1 & 1 & 1 & 1 & 1 \\
\hline
\end{tabular}

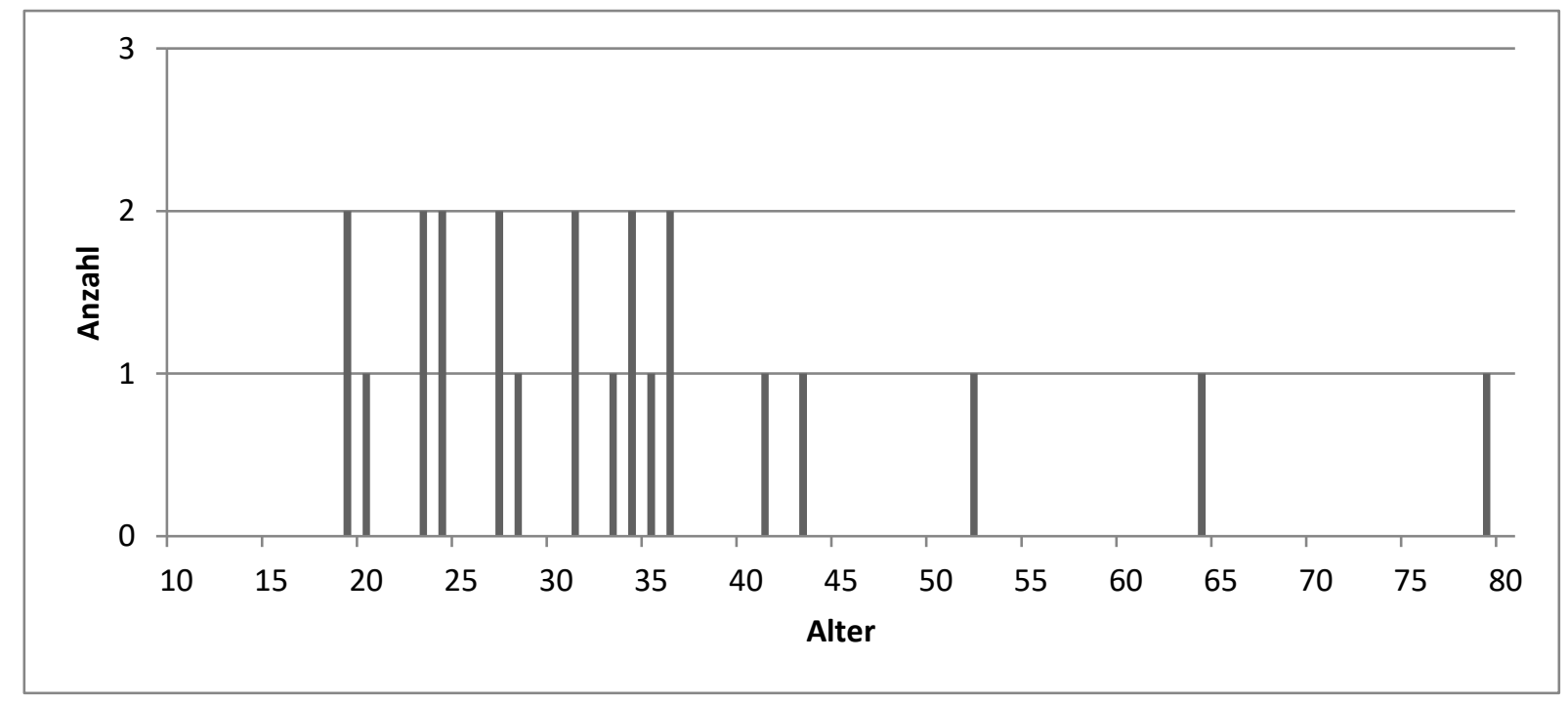

Abbildung 2: Altersverteilung der Amokfahrer 
Von den 23 verifizierten Amokfahrern konnten aus dem zur Verfügung stehenden Material (Pressemitteilungen) folgende Informationen gewonnen werden: Bei 43,5 \% $(n=10)$ fanden sich keine Informationen zum Familienstand, 17,4 \% $(n=4)$ waren verheiratet, 17,4 \% $(\mathrm{n}=4)$ waren ledig, 8,7\% $(\mathrm{n}=2)$ lebten in Trennung, $13 \%(\mathrm{n}=3)$ in einer nichtehelichen Lebensgemeinschaft.

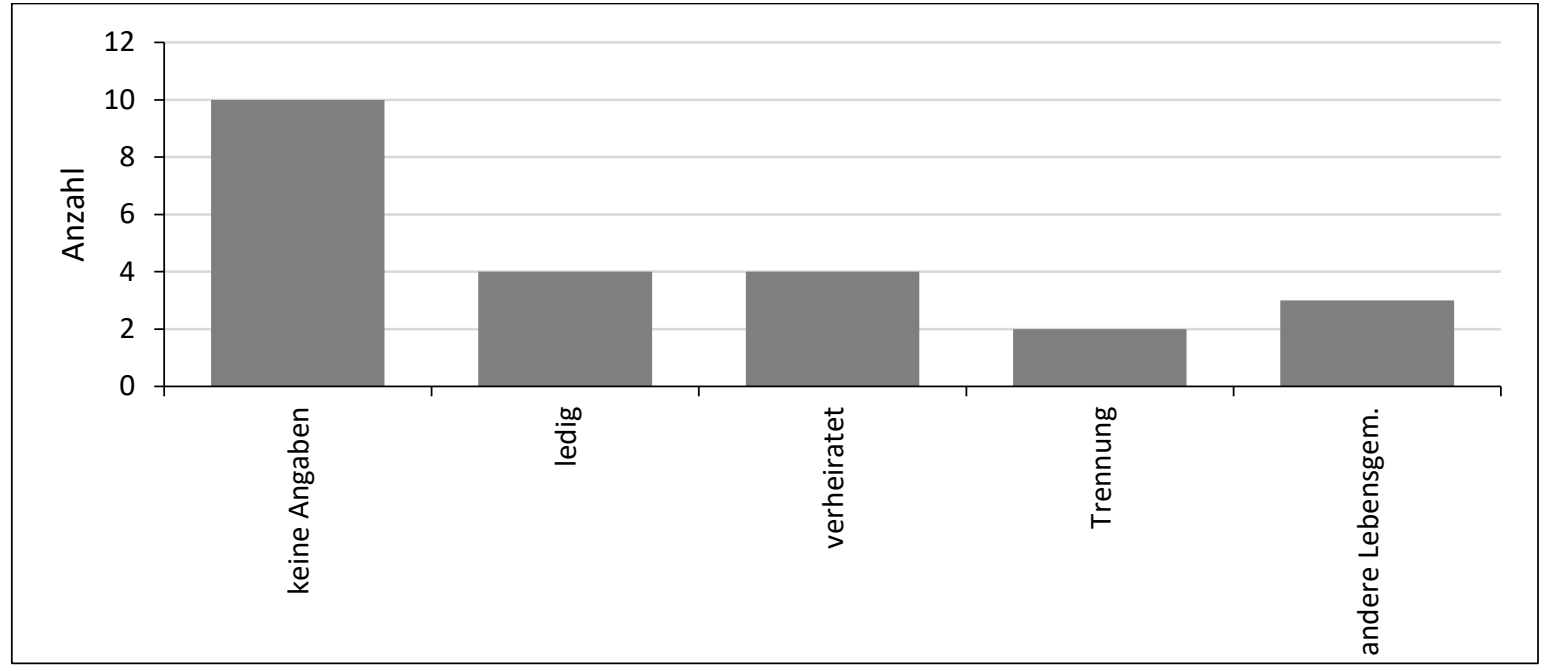

Abbildung 3: Familienstand der Amokfahrer

In 52,2 \% der Fälle $(\mathrm{n}=12)$ fanden sich in den ausgewerteten Presseartikeln keine Angaben zur Berufsausbildung des Amokfahrers. Bei 21,7 \% war zu erfahren, dass sie Arbeiter und Handwerker waren (blue collar, $\mathrm{n}=5$ ), $13 \%$ waren Angestellte (white collar , $\mathrm{n}=3$ ) und 8,7 \% hatten einen akademischen Abschluss $(n=2)$. 


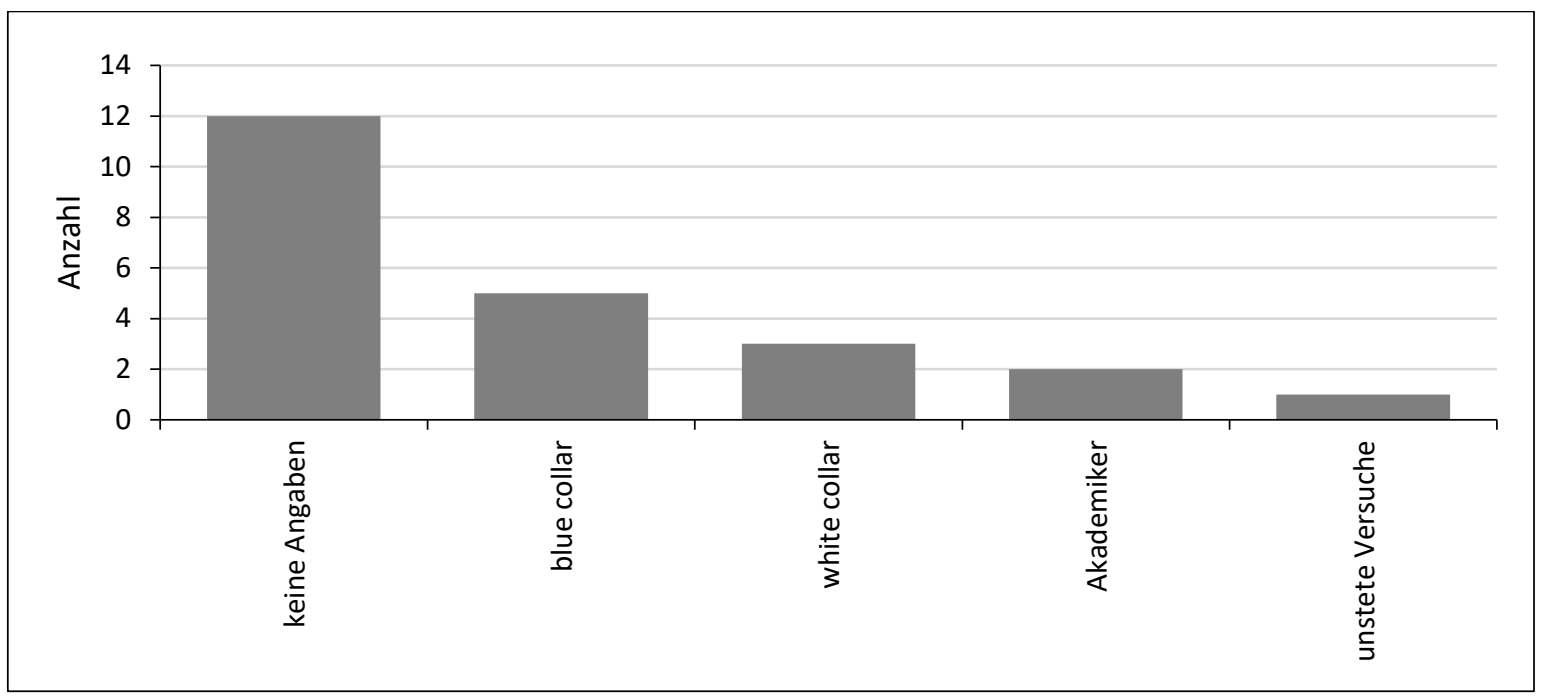

Abbildung 4: Berufsausbildung der Amokfahrer

In 43,5 \% der Fälle $(n=10)$ enthielten die ausgewerteten Presseartikel keine Angaben, ob der Amokfahrer vor seiner Tat in einem Beschäftigungsverhältnis stand. Von den Amokfahrern, zu denen Informationen es Informationen gab, waren 30,4 \% $(\mathrm{n}=7)$ arbeitslos und $8,7 \%(\mathrm{n}=2)$ ohne eine feste Beschäftigung (nur unregelmäßige Jobs). Nur 8,7\% von ihnen $(n=2)$ hatten eine feste Anstellung, weitere $8,7 \%(n=2)$ waren Rentner.

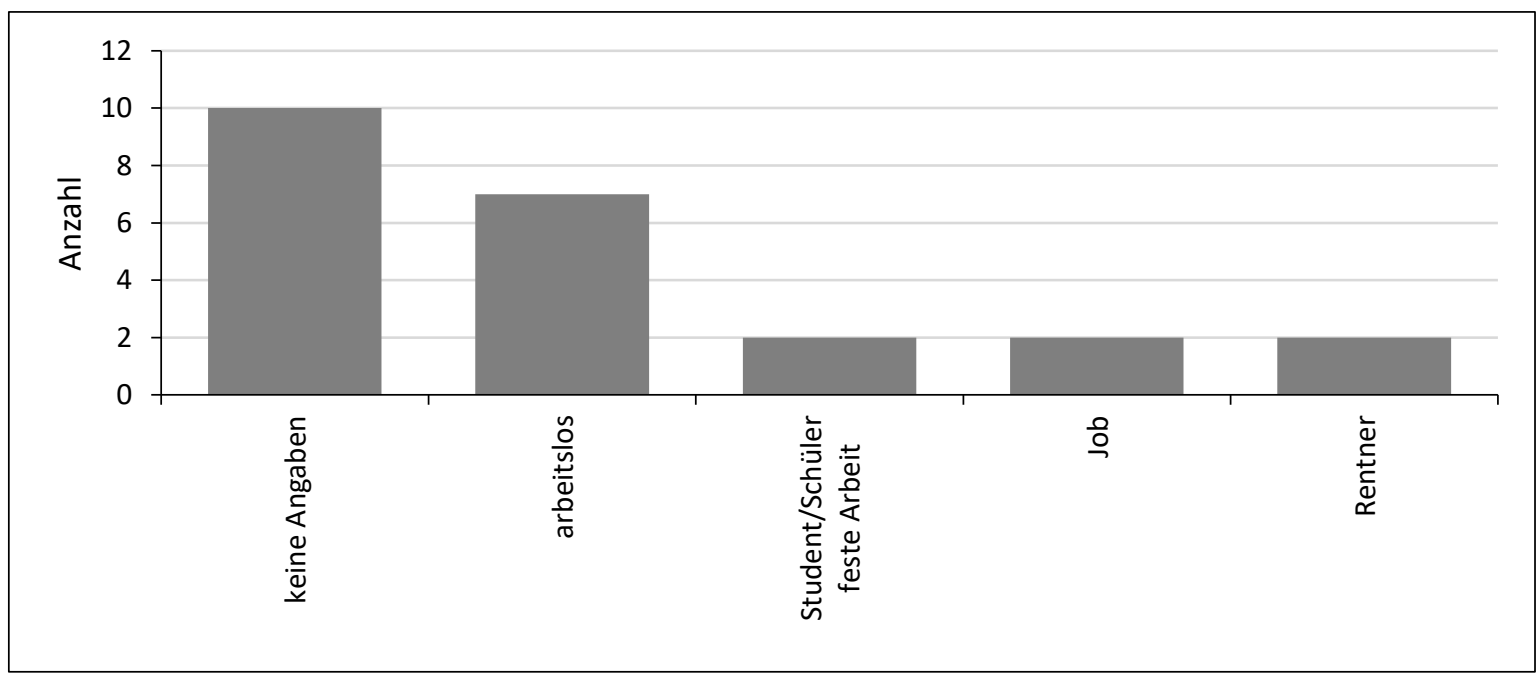

Abbildung 5: Beschäftigungsverhältnis der Amokfahrer 
In etwas weniger als der Hälfte der Fälle $(47,8 \%, \mathrm{n}=11)$ standen aus den ausgewerteten Presseartikeln keine Angaben über Persönlichkeitsauffälligkeiten der Amokfahrer zur Verfügung. Bei etwas mehr als der Hälfte $(52,2 \%, \mathrm{n}=12)$ gab es hierzu Angaben, in einem Fall (4,3 \%) werden Persönlichkeitsauffälligkeiten ausdrücklich verneint. Bei den anderen Fällen $(47,8 \%, \mathrm{n}=11)$ wurden Persönlichkeitsauffälligkeiten wie querulatorische, hypochondrische, paranoide oder anderweitig wahnhafte Auffälligkeiten $(8,7 \%$ der Fälle, $\mathrm{n}=2)$, weitere $17,4 \%$ der Amokfahrer $(\mathrm{n}=4)$ wurden als impulsiv und unbeherrscht beschrieben. Zwei spätere Amokfahrer (8,7\%) waren bereits im Vorfeld durch schlimmen bedrohlichen Jähzorn aufgefallen. Eine Beschreibung der Täterpersönlichkeit, die nicht explizit auf die Persönlichkeitsauffälligkeiten abzielte, fand sich lediglich in einem Fall eines Amokfahrers.

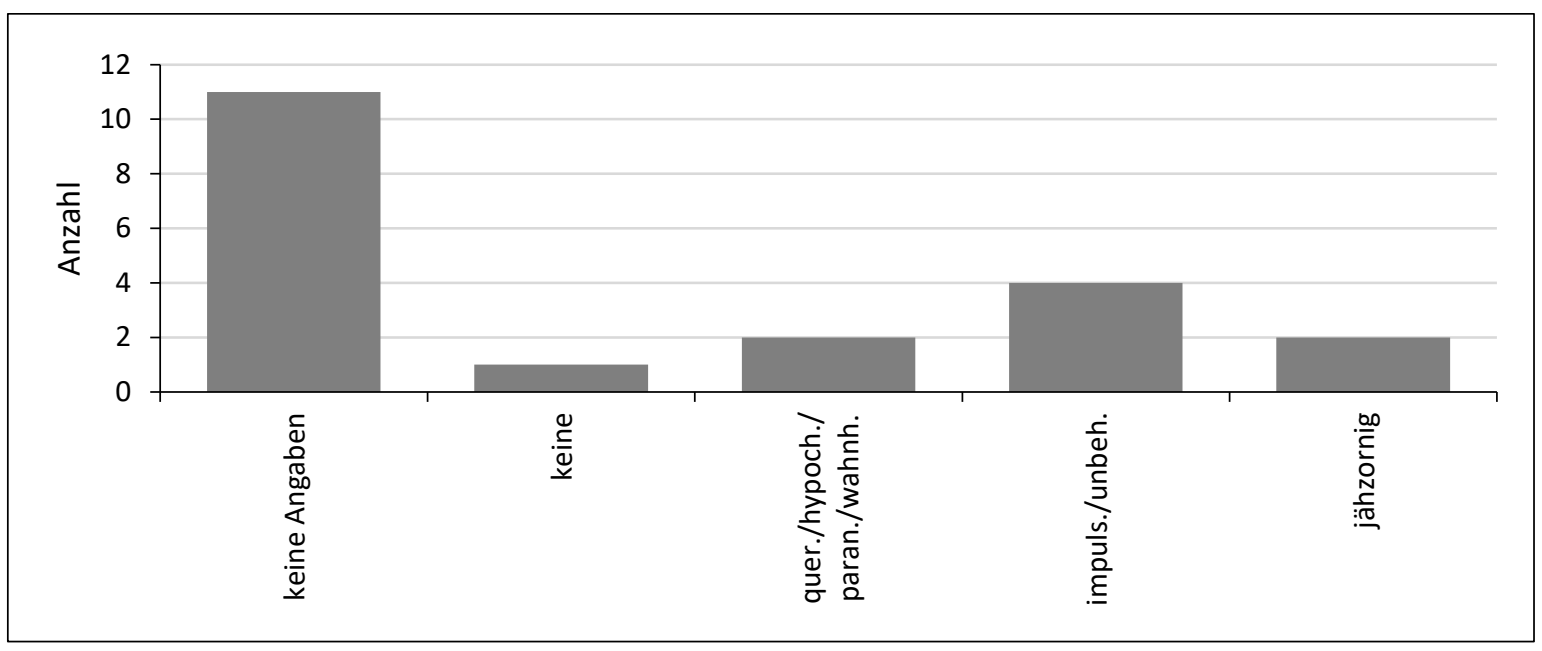

Abbildung 6: Persönlichkeitsauffälligkeiten

In 78,3 \% der Fälle $(\mathrm{n}=18)$ fanden sich hierzu in den ausgewerteten Zeitungsartikeln keine Angaben. Nur bei 21,7 \% der Fälle $(n=5)$ wurde berichtet, dass bei dem Amokfahrer schon vor seiner Tat eine schwerwiegende psychiatrische Erkrankung bestand. 


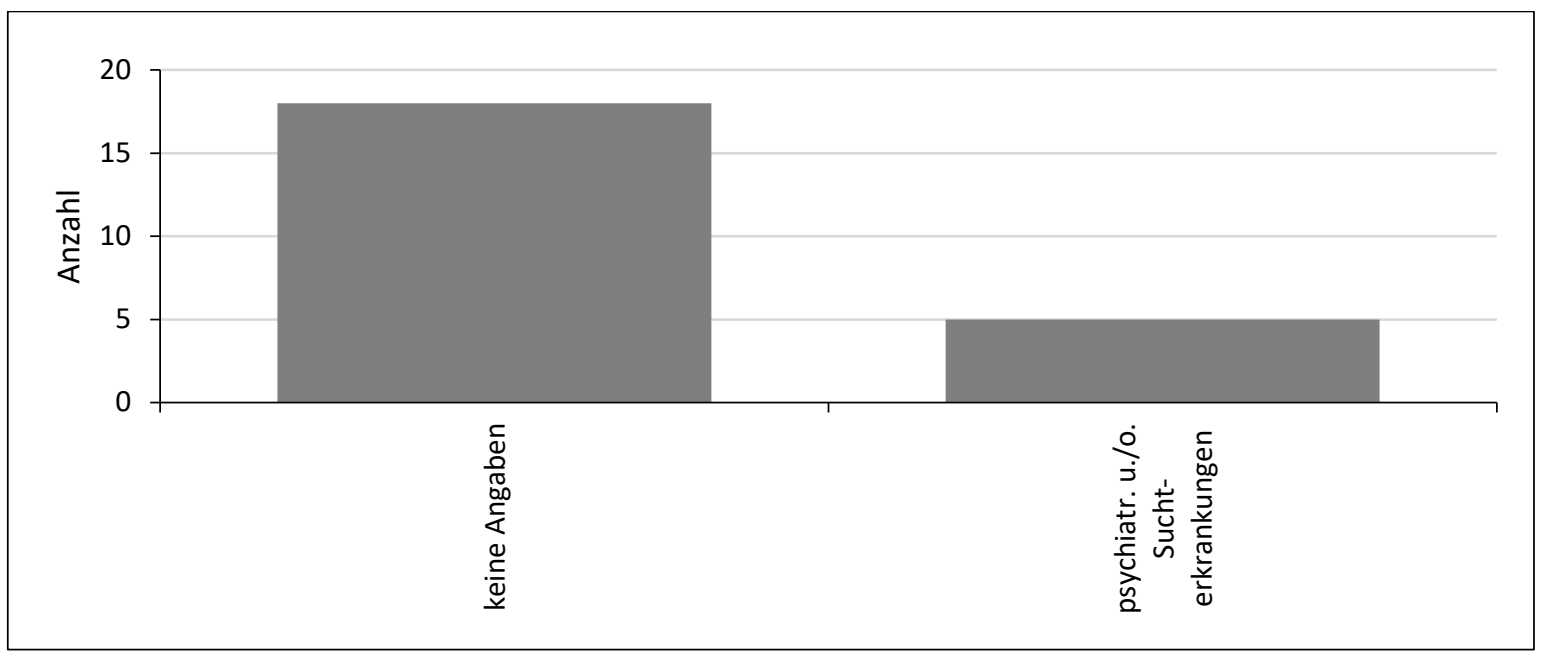

Abbildung 7: Psychiatrische u./o. Suchterkrankung vor der Tat

Bei 69,6\% der untersuchten Amokfahrer $(\mathrm{n}=16)$ wurde aus den ausgewerteten Presseartikeln keine andere kriminelle Handlung im Vorfeld der Tat bekannt. Allerdings hatten $30,4 \%$ von ihnen $(n=7)$ vor ihrer Amokfahrt kriminelle Handlungen begangen.

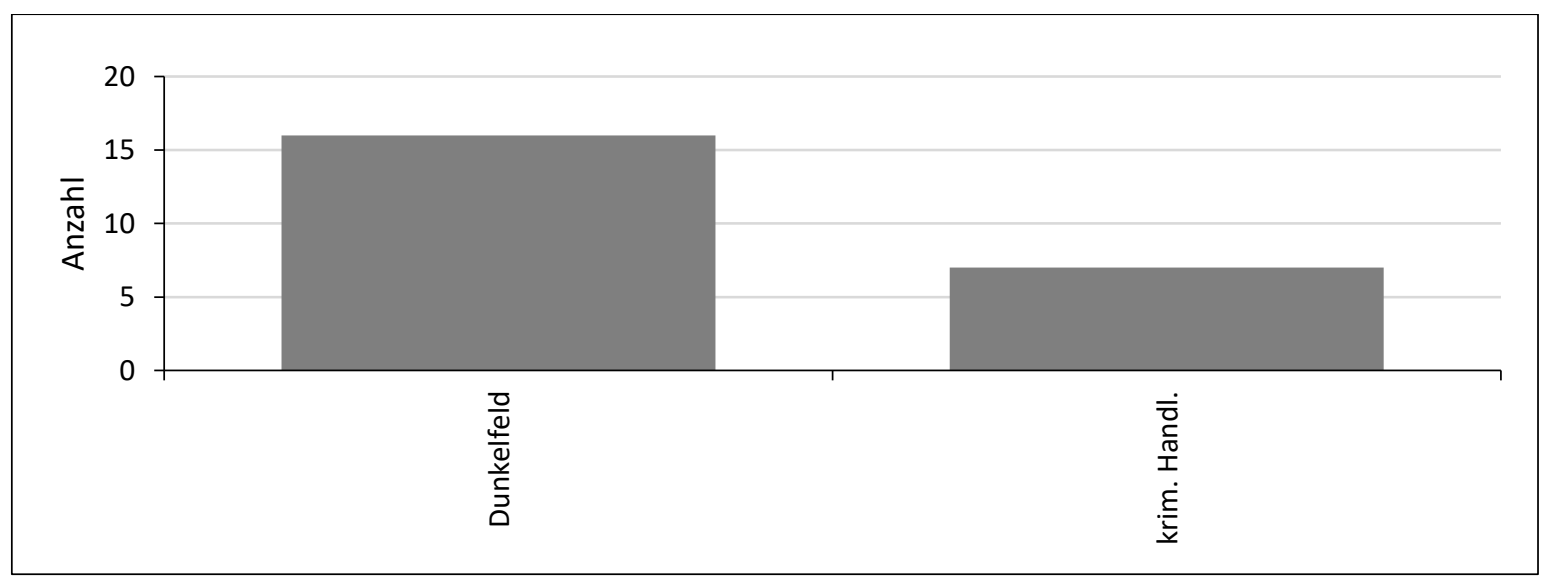

Abbildung 8: Tatvorfeld

95,7 \% der Amokfahrer dieser Untersuchung $(\mathrm{n}=22)$ waren Männer. Nur in einem Fall $(4,3 \%)$ handelte es sich um eine Amokfahrerin. 


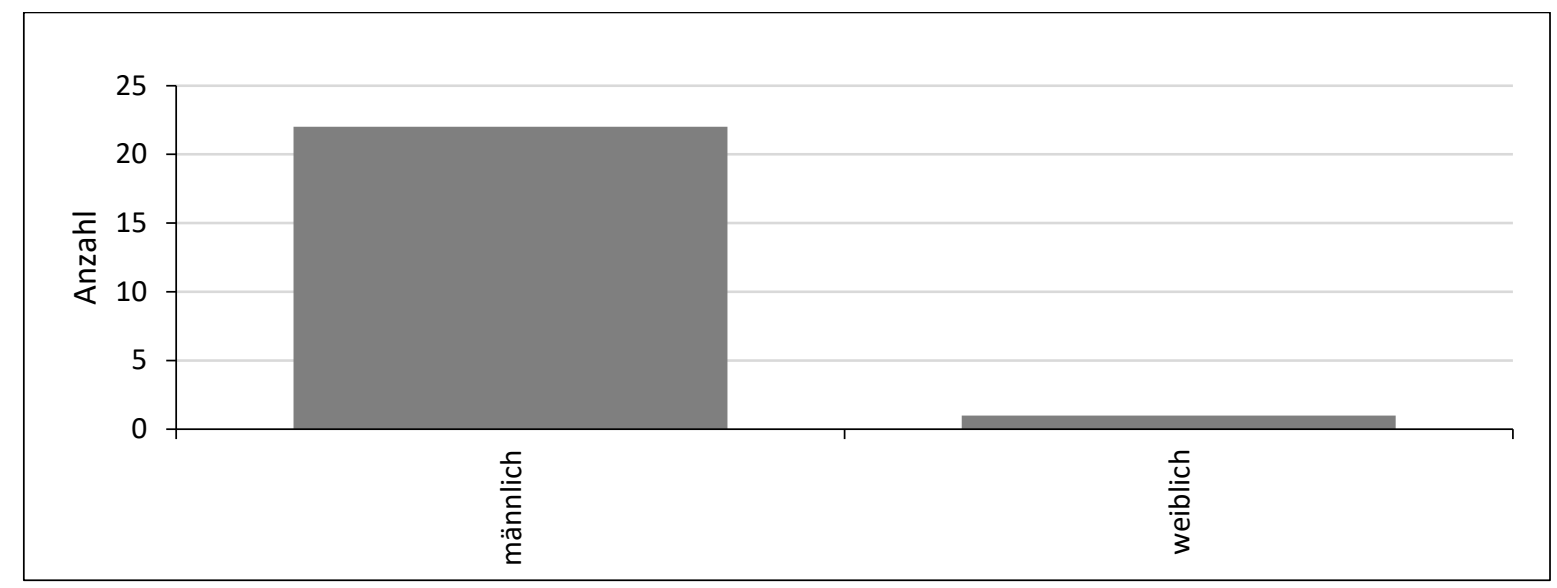

Abbildung 9: Geschlechtsverteilung

Von den Amokfahrern auf bundesdeutschen Straßen, über die in der deutschen Presse berichtet wurde, waren 69,6 \% Deutsche $(n=16), 26,1 \%$ der Täter $(n=6)$ stammten aus einem Land der Europäischen Union und nur ein Fahrer (4,3 \%) hatte eine außereuropäische Herkunft.

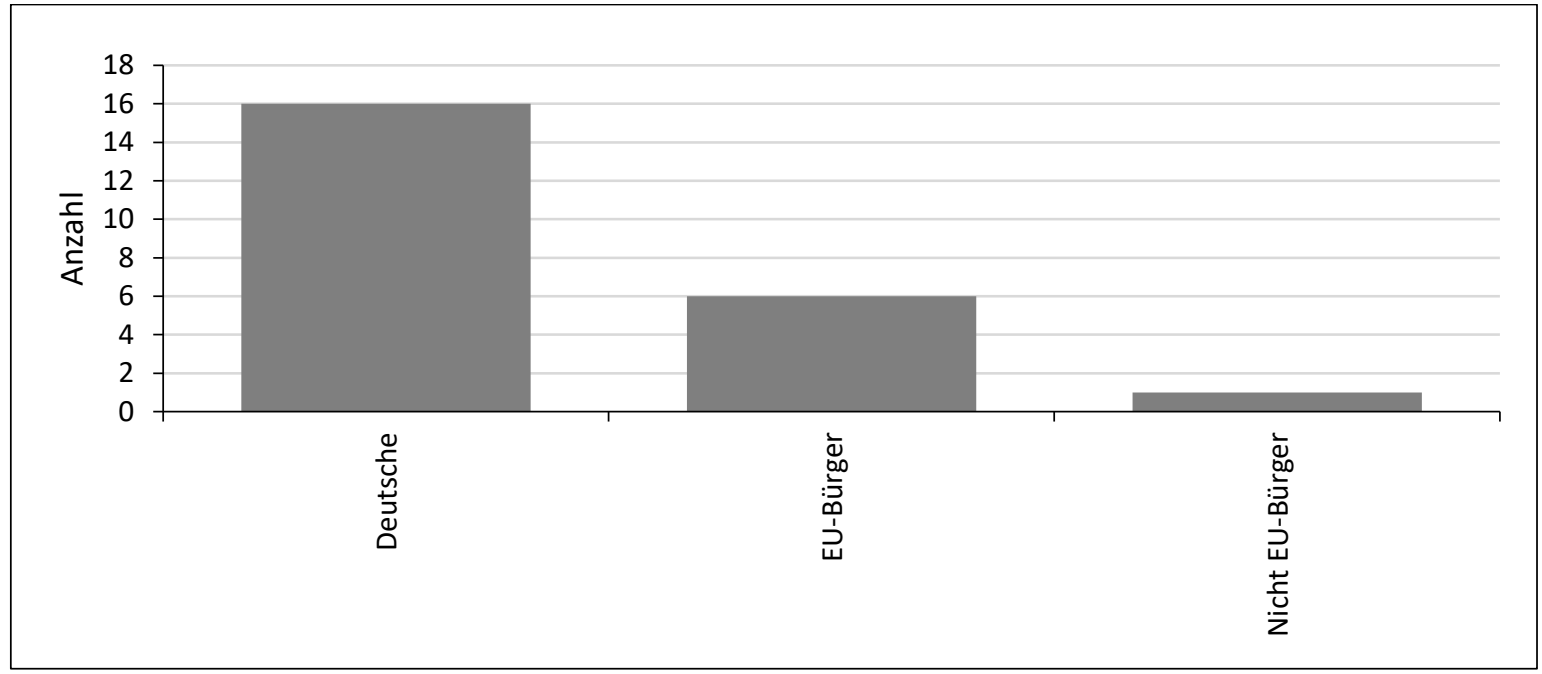

Abbildung 10: Herkunft der Amokfahrer

$\mathrm{Zu}$ den Tatmotiven fanden sich in den Presseartikeln über Amokfahrten die meisten Angaben. Nur in vier Fällen (17,4 \%) gab es hierzu überhaupt keine Angaben. Bei den anderen 18 Amokfahrern (82,6 \%) waren tatauslösende Motive Wohnungsverlust 4,3\% $(\mathrm{n}=1)$, Verlust des Arbeitsplatzes 4,3\% $(\mathrm{n}=1)$, Niederlage bei einer Auseinandersetzung vor 
Gericht 4,3\% $(\mathrm{n}=1)$, andere schwerwiegende Lebenskonflikte $13 \%(\mathrm{n}=3)$ bzw. Ehescheidung /Partnerschaftstrennung 17,4 \% $(\mathrm{n}=4)$. Bei weitern 39,1\% der Amokfahrer $(\mathrm{n}=9)$ war ein tatauslösendes Motiv bekannt, stand aber in keinem nachvollziehbaren Verhältnis zur Schwere der von ihnen begangenen Tat. In diesem Fällen ist durchaus möglich, dass, auch wenn hier keine psychiatrische Vorerkrankung bekannt war, ein Wahnmotiv handlungsleitend war.

Über das sonstige Vortatverhalten der Amokfahrer liegen in 34,8 \% der gesicherten Amokfälle $(n=8)$ keine Angaben vor. Von 21,7\% der Amokfahrer $(n=5)$ wurde berichtet, dass sie ein aggressives Vortatverhalten zeigten, bei weiteren $21,7 \%$ war äußerlich keine aggressive Affektspannung vor der Tat erkennbar. Und bei den übrigen 21,7\% der bestand kein motivationaler Zusammenhang zwischen ihrem Verhalten vor der Tat und ihrer Amokfahrt.) 


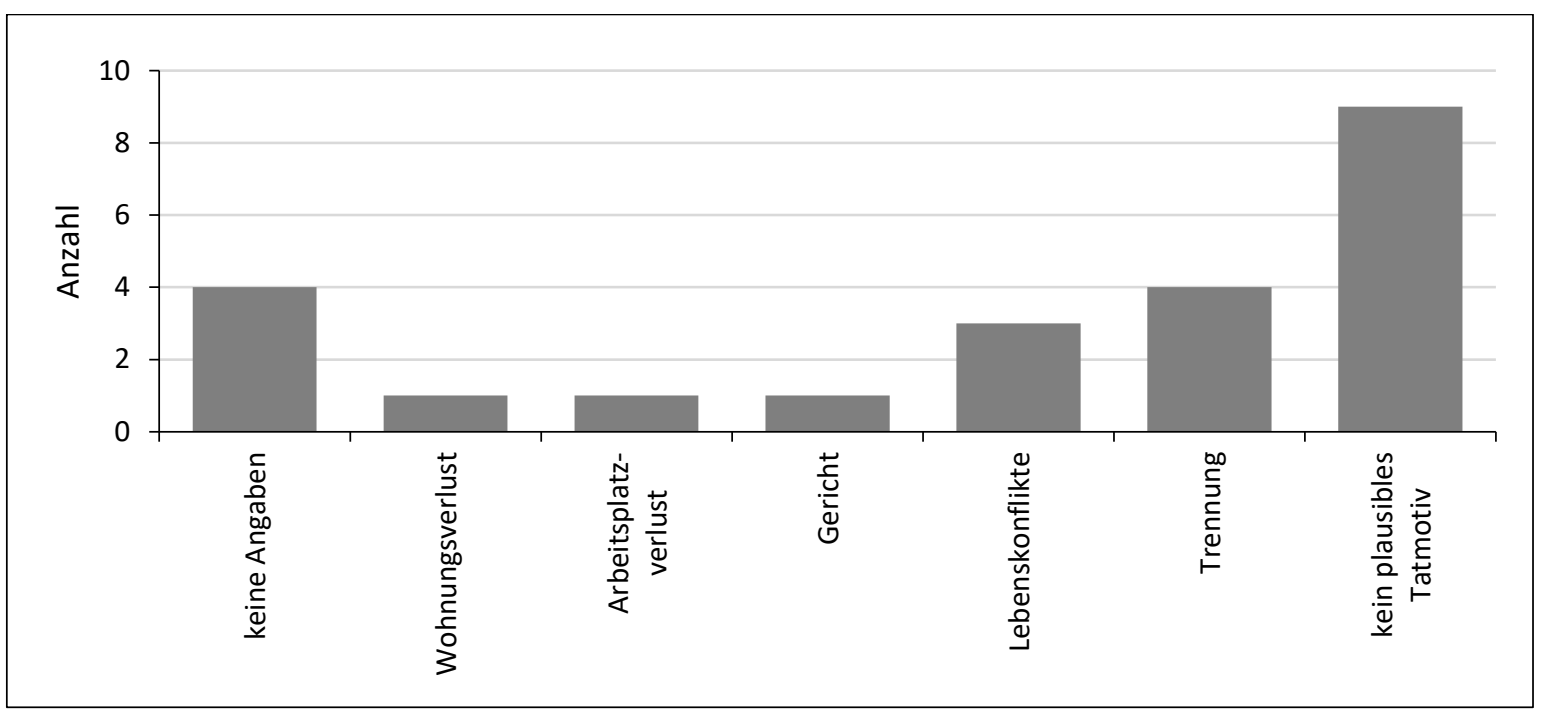

Abbildung 11: Tatmotive

In 52,2 \% der Fälle $(\mathrm{n}=12)$ war das Tatmotiv für die Amokfahrt sofort handlungseinleitend. Nur in jeweils einem Fall lagen zwischen dem Kränkungs- oder Bedrohungsereignis, das zum Tatmotiv der späteren Amokfahrt wurde, eine Latenzzeit von Tagen bzw. Monaten. In einem Fall erfolgte die Tat sogar noch vor dem befürchteten Ereignis, das das hier das Tatmotiv darstellte.

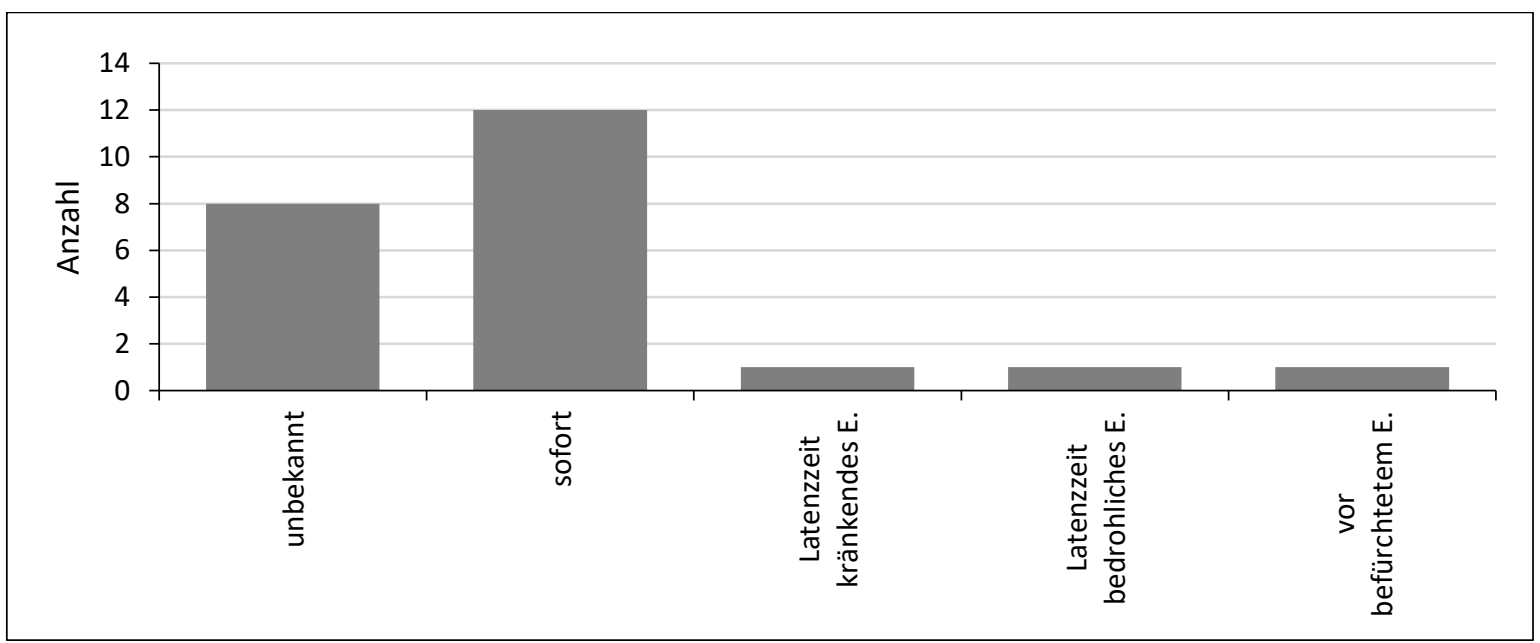

Abbildung 12: Zeit-Tatmotiv-Beziehung 
Bei 73,9 \% der Amokfahrten $(n=17)$ die ihm unbekannten Tatopfer wahllos während der Fahrt. Mit seinem Fahrzeug. Bei weiteren $13 \%$ der Amokfahrten $(\mathrm{n}=3)$ war die Tat ausschließlich auf eine umschriebene Menschengruppe gerichtet, in die der Amokfahrer hineinfuhr. Bei weiteren $13 \%$ der Amokfahrten $(n=3)$ kam es nach anfänglicher Gerichtetheit auf eine Menschengruppe sekundär zu einer wahllosen Ausdehnung der Amokfahrt auf zufällige Tatopfer. Bei $91.3 \%$ der untersuchten Amokfahrten $(n=21)$ bestand von vornherein keine persönliche Täter-Opfer-Beziehung. Nur in je einem Fall (4,3\%) bestand zu Beginn der Amokfahrt zwischen dem späteren Amokfahrer und einen Tatopfern eine persönliche Täter-Opfer-Beziehung: Im einem Fall war das Opfer der Amokfahrt die Partnerin des Täters, im zweiten Fall waren es Mitglieder der eigenen Familie des Amokfahrers.

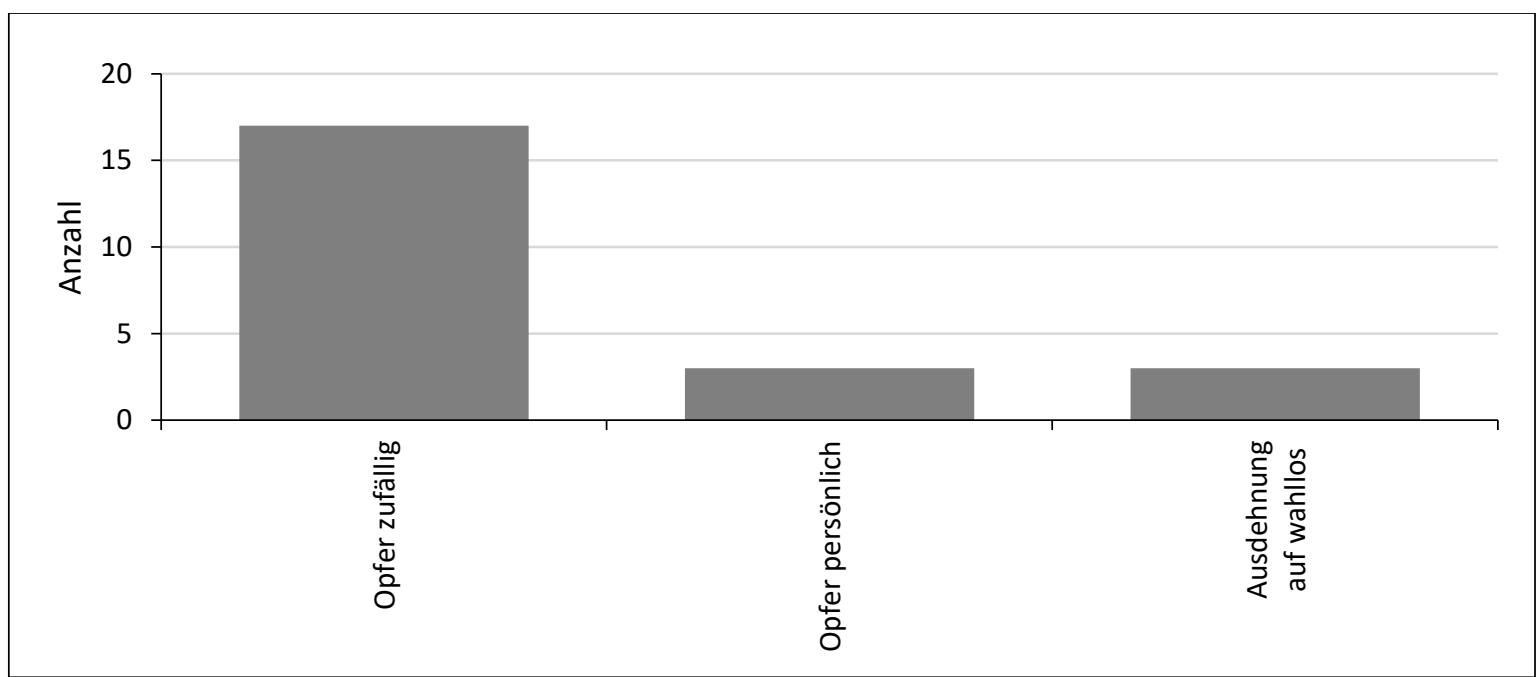

Abbildung 13: Konfliktbezogene. Täter-Opfer Beziehung

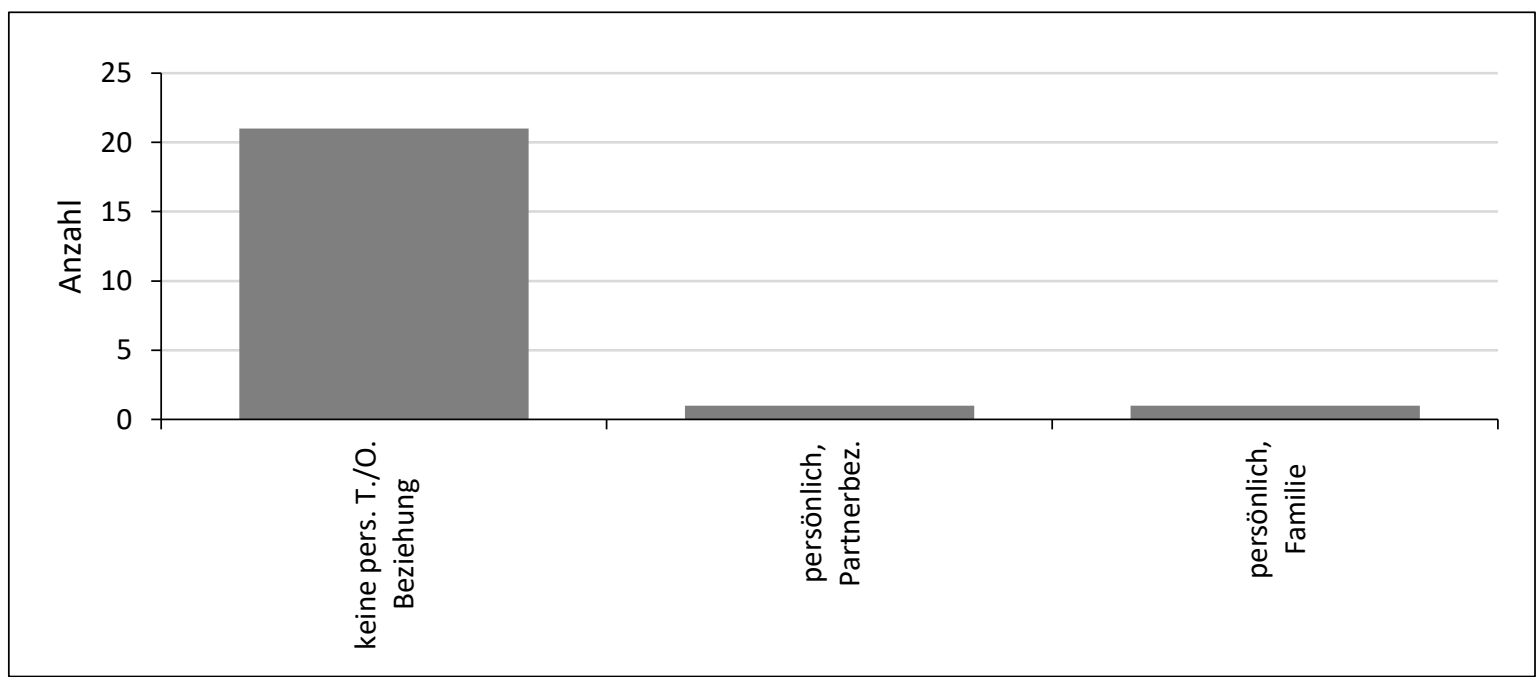

Abbildung 14: Persönliche Täter-Opfer Beziehung 
Sich ein Auto als Tatwaffe für eine Amokfahrt zu beschaffen ist - zumindest in Europa wesentlich leichter, als an eine Schusswaffe für einen Amoklauf zu kommen. In Deutschland ist heutzutage fast jeder Erwachsene im Besitz einer Fahrerlaubnis und es gibt kaum noch einen Haushalt, in dem nicht mindestens ein motorisiertes Fahrzeug stets fahrbereit zur Verfügung steht. Somit wäre die Durchführung einer Amokfahrt mit einem motorisierten Fahrzeug - technisch und logistisch gesehen für eine sehr große Personenzahl denkbar einfach und bedürfte nicht einmal der Beschaffung einer Tatwaffe.

Der Tatbeginn der in dieser Arbeit untersuchten Amokfahrten erscheint ungefähr gleichmäßig über einen Zeitraum von 24 Stunden verteilt mit einem 1. Gipfel in den Vormittagsstunden und einem 2. kleineren Gipfel am Nachmittag. In 21,7\% der Fälle $(n=5)$ lagen keine Angaben zum Tatbeginn vor.

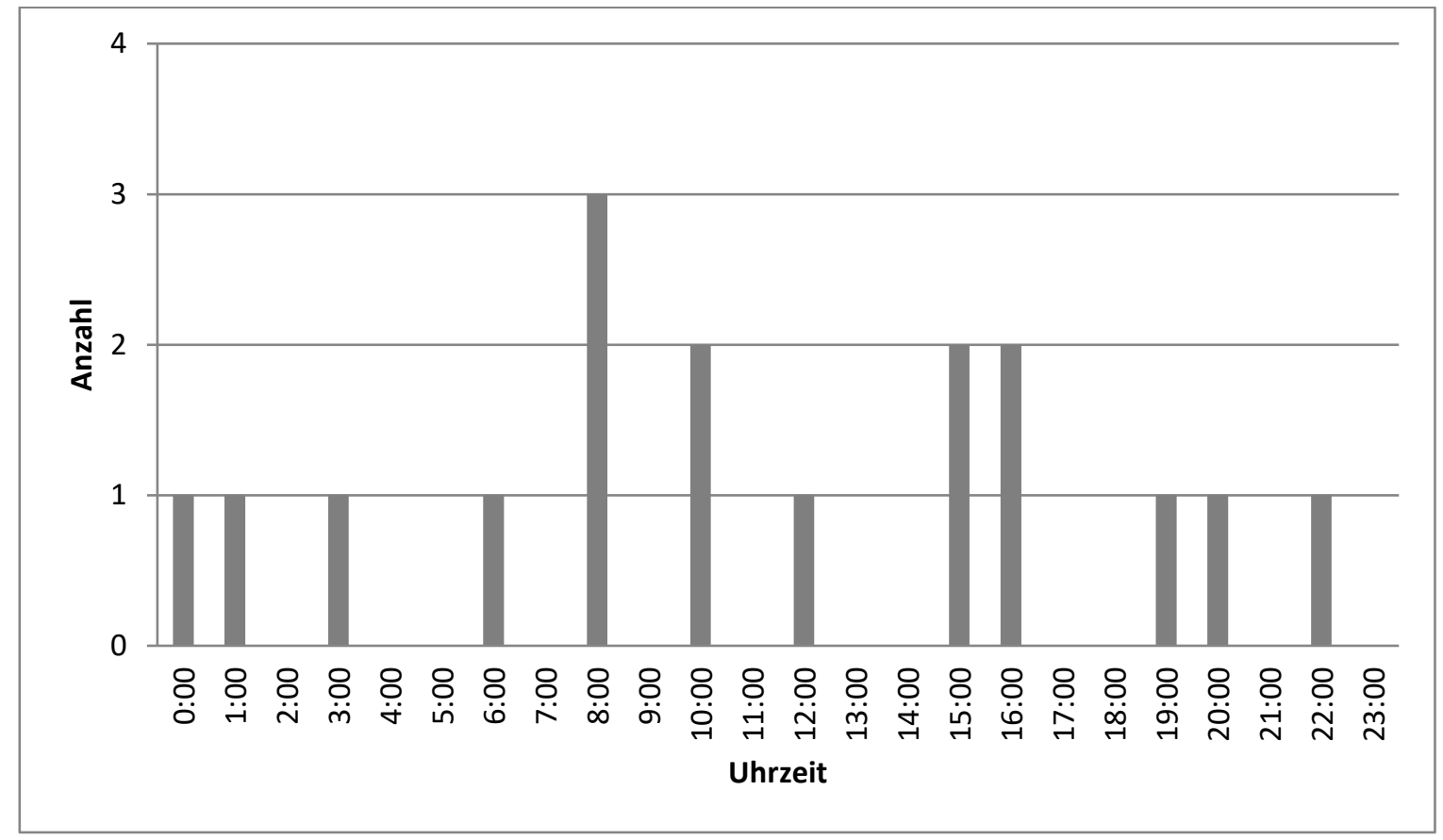

Abbildung 15: Anzahl der Fahrten pro Uhrzeit 
Von den hier untersuchten Amokfahrten dauerten 56,5\% $(\mathrm{n}=13)$ nicht länger als eine Stunde. Nur in je einem der Fälle (4,3\%) dauerte die Amokfahrt zwei, vier oder gar fünf Stunden. Bei 30,4 \% der untersuchten Amokfahrten $(n=7)$ waren keine Angaben zur Dauer der Amokfahrt vorhanden.

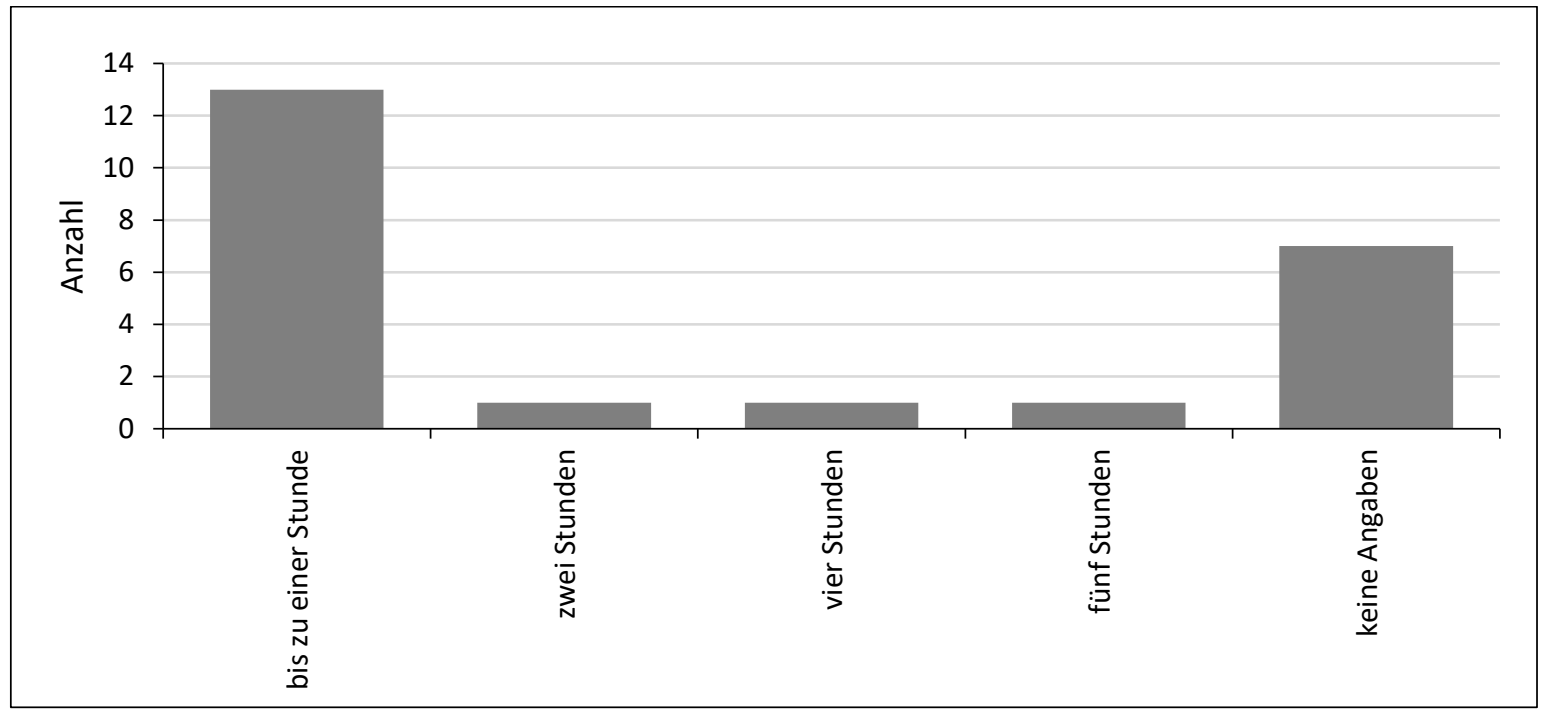

Abbildung 16: Tatdauer

Bei 30,4 \% der untersuchten Amokfahrten $(n=7)$ begann und endete die Tat an einem umschriebenen Tatort. In 69,6 \% der Fälle $(\mathrm{n}=16)$ begann die Amokfahrt an einem umschriebenen Tatort, der sich im weiteren Tatverlauf erheblich räumlich ausdehnte.

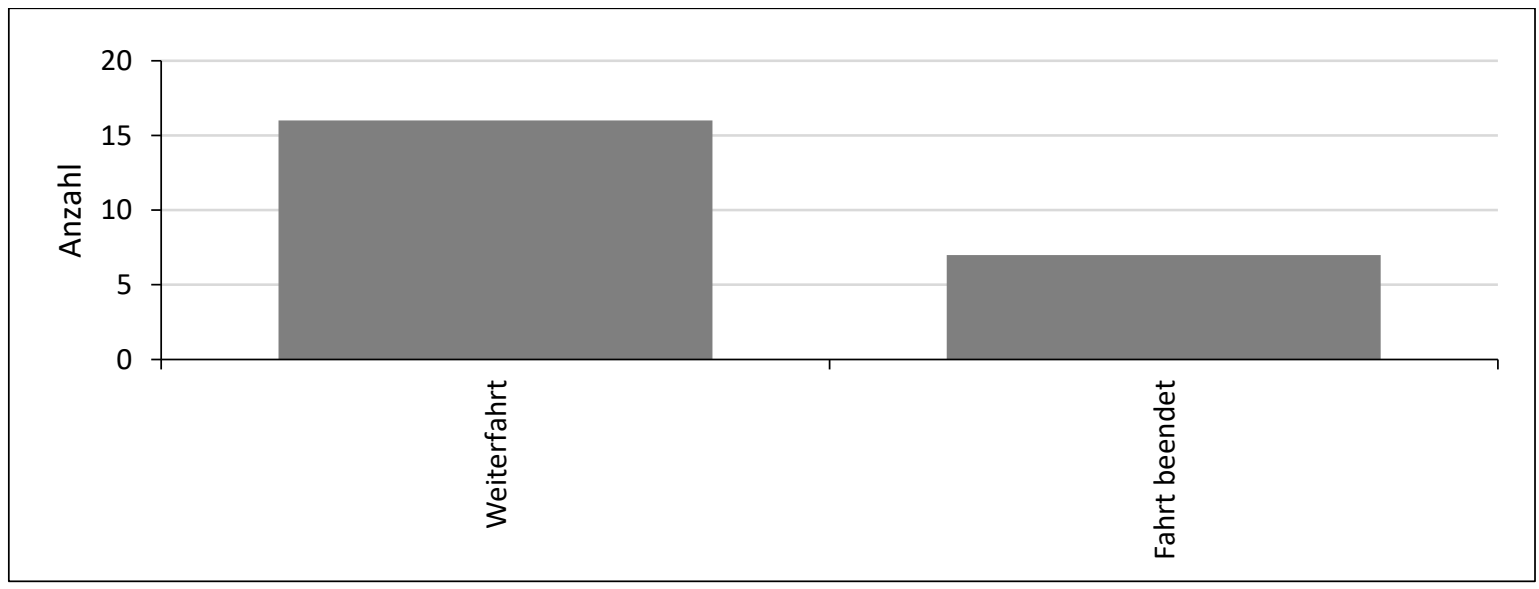

Abbildung 17: Tatortwechsel 
Bei 52,2\% der untersuchten Amokfahrten $(\mathrm{n}=12)$ gab es keine Todesopfer, bei weiteren $34,8 \%$ der untersuchten Amokfahrten $(n=8)$ gab es ein Todesopfer. In 2 Fällen $(8,7 \%)$ wurden durch die Amokfahrt drei Menschen getötet und in einem Fall (4,3 \%) forderte eine Amokfahrt sogar fünf Todesopfer.

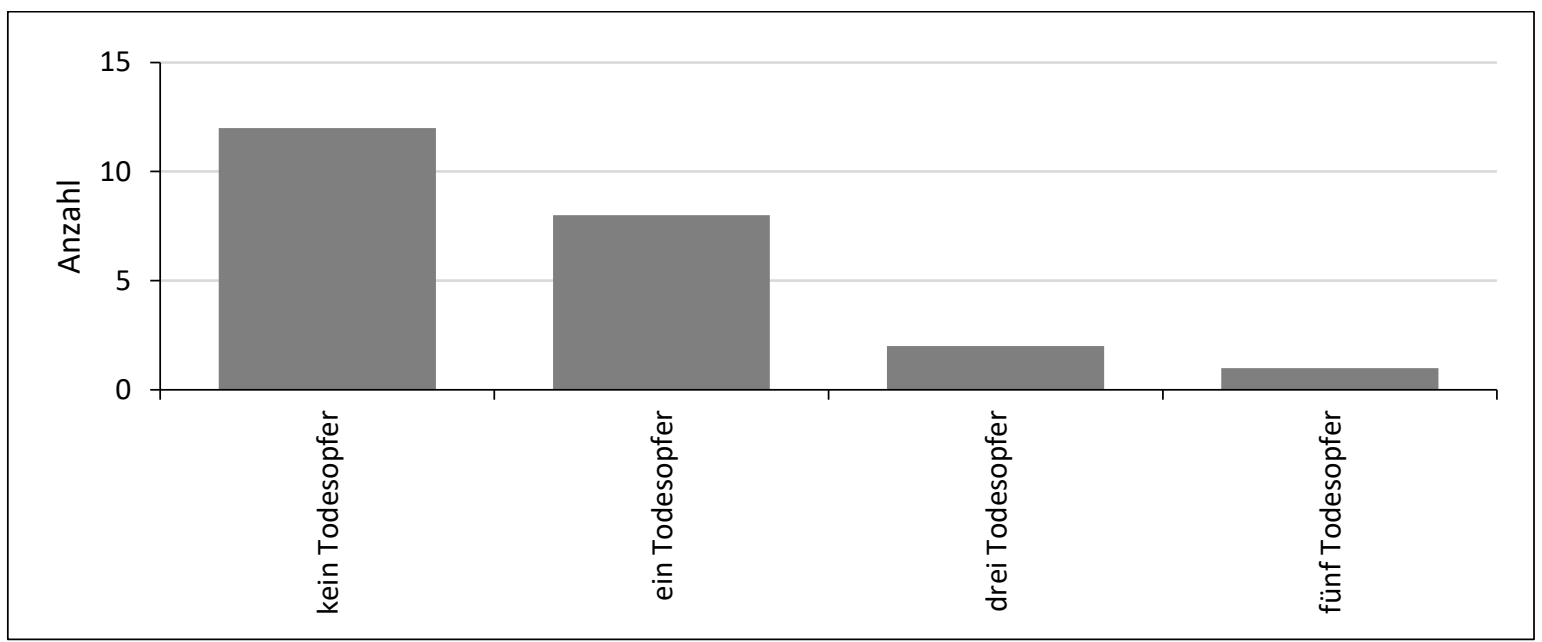

Abbildung 18: Todesopferzahl

Bei vier Amokfahrten (17,4 \%) gab es keine Verletzten, bei sechs weiteren (26,1\%) wurde jeweils eine Person verletzt. Bei zwei der untersuchten Amokfahrten (8,7 \%) wurden jeweils zwei Personen verletzt, bei einer weiteren Amokfahrt (4,3\%) drei Personen. Bei drei weiteren Amokfahrten (13,0 \%) gab es vier Verletzte, in je einem der folgenden Fälle wurden durch einen Amokfahrer fünf bzw. sechs Personen verletzt. In mehreren Fällen forderten Amokfahrten noch höhere Opferzahlen - einem Fall verletzte ein Amokfahrer 14 Personen, in zwei weiteren Fällen gab es nach einer Amokfahrt 15 Verletzte in einem Fall sogar 26 Verletzte. 


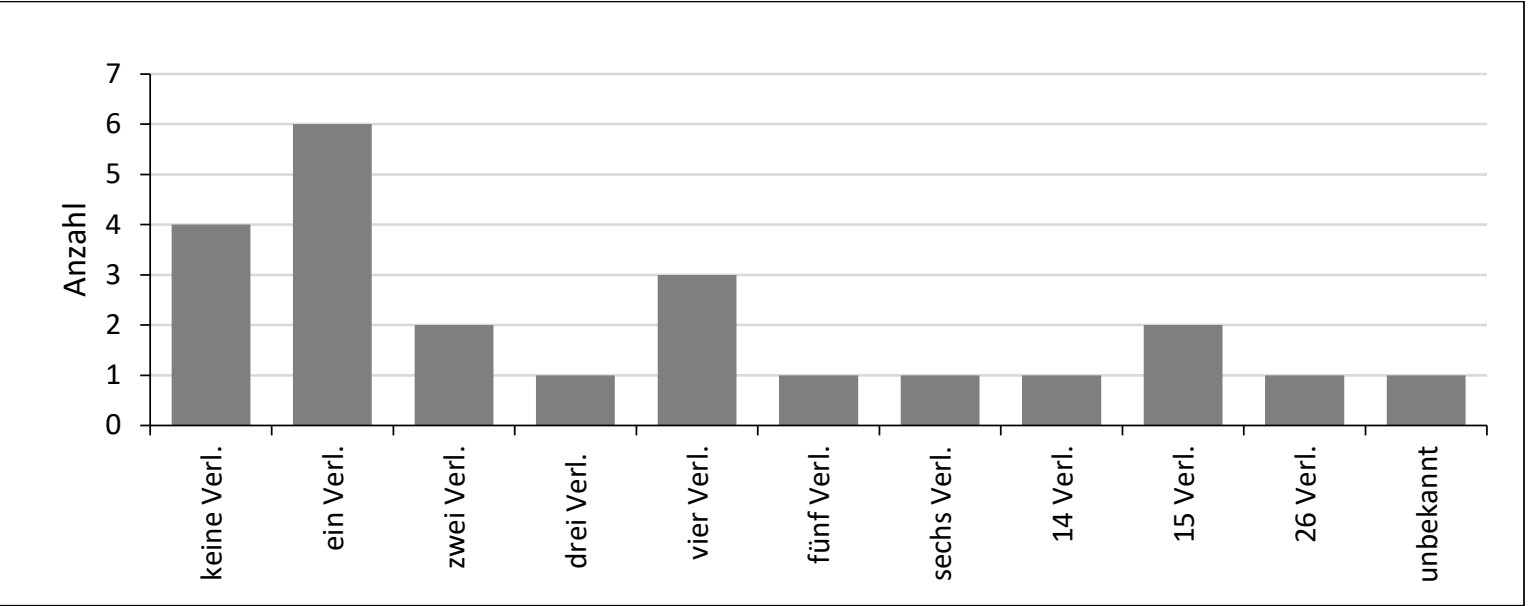

Abbildung 19: Verletztenzahl pro Fall

In zwei Fällen (8,7 \%) konnte das Geschlecht der Tatopfer nicht ermittelt werden. In weiteren neun Fällen (39,1 \%) waren die Opfer überwiegend männlich, in fünf Fällen (21 7 \%) überwiegend weiblich und in sieben Fällen (30,4 \%) gemischt männlich und weiblich.

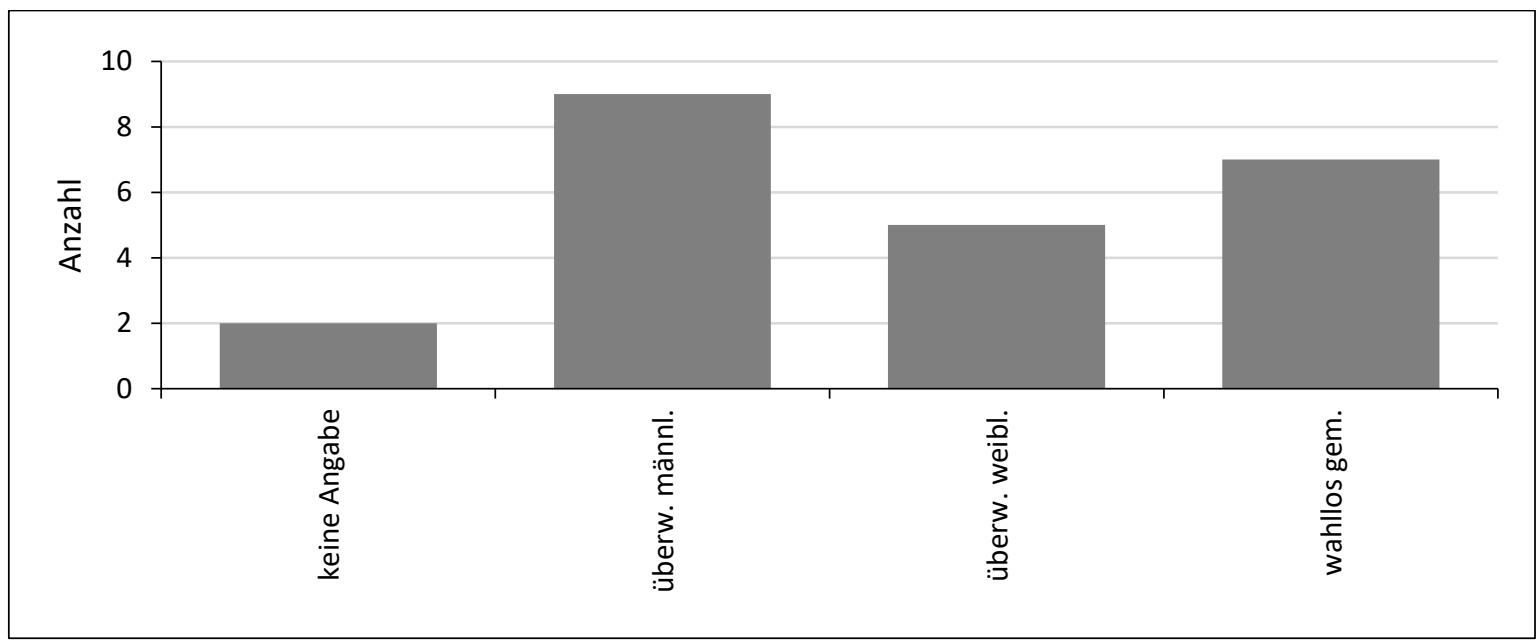

Abbildung 20: Opfergeschlecht

In zwei Fällen (8,7 \%) gab es keine Angaben zum Alter der Opfer der Amokfahrt. In 16 Fällen (69,6 \%) handelte es sich bei den Tatopfern überwiegend um Erwachsene, bei fünf weiteren Fällen (21,7 \%) waren die Opfer vom Alter her wahllos gemischt. 


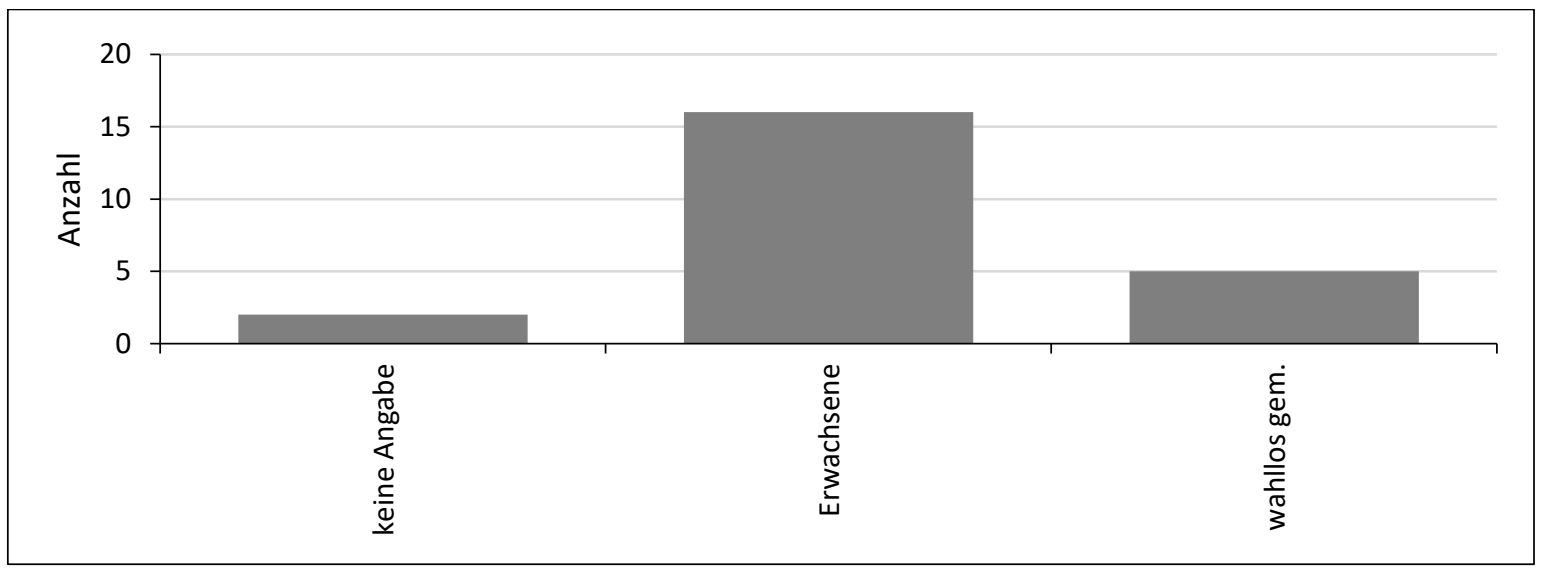

Abbildung 21: Opferalter

Bei 18 von 23 Amokfahrten (78,3 \%) richteten die Täter erhebliche Sachschäden an. In fünf Fällen (21,7 \%) wurde durch die Amokfahrt kein oder nur ein geringer Sachschaden angerichtet (z.B. Blechschäden), weil es dem Unfallgegner gelang, dem Amokfahrer im letzten Moment auszuweichen.

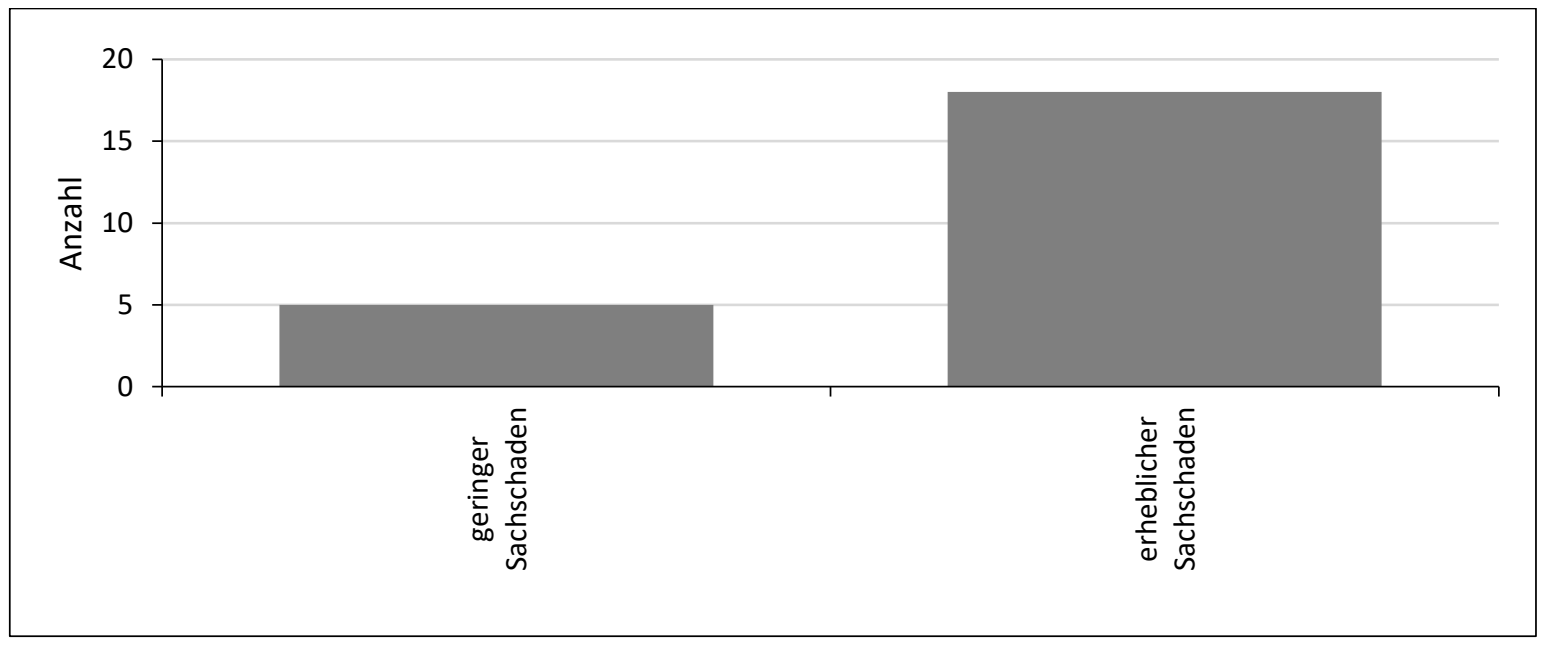

Abbildung 22: Sachschaden

Um die Amokfahrt zu beenden, musste in den meisten Fällen der Amokfahrer von der Polizei gestoppt werden. Allerdings begingen in dieser Untersuchung 82,6 \% der Amokfahrer $(\mathrm{n}=19)$ vor ihrer Ergreifung durch die Polizei einen Fluchtversuch, Lediglich 17,4\% $(\mathrm{n}=4)$ ließen sich am Ende der Amokfahrt widerstandslos von der Polizei festnehmen. 


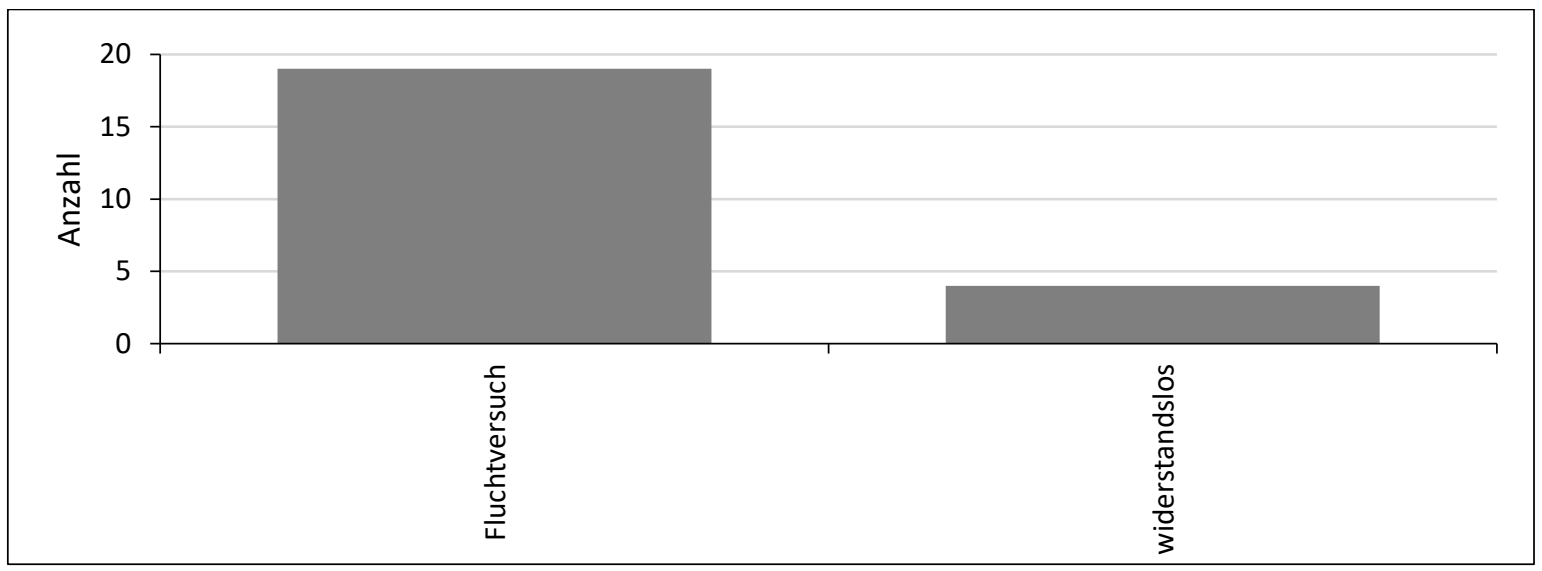

Abbildung 23: Fluchtverlauf

Um ihre Amokfahrt zu beenden, mussten 43,5 \% der Amokfahrer $(n=10)$ dieser Untersuchung von der Polizei überwältigt und gewaltsam entwaffnet werden. Nur vier von ihnen $(17,4 \%)$ ließen sich, nachdem die Polizei sie gestoppt hatte, widerstandslos festnehmen. Ein Täter (4,3 \%) stellte sich selbst der Polizei, weiter acht Amokfahrer (34,8 \%) begingen am Ende ihrer Tat einen Suizidversuch oder vollendeten Suizid.

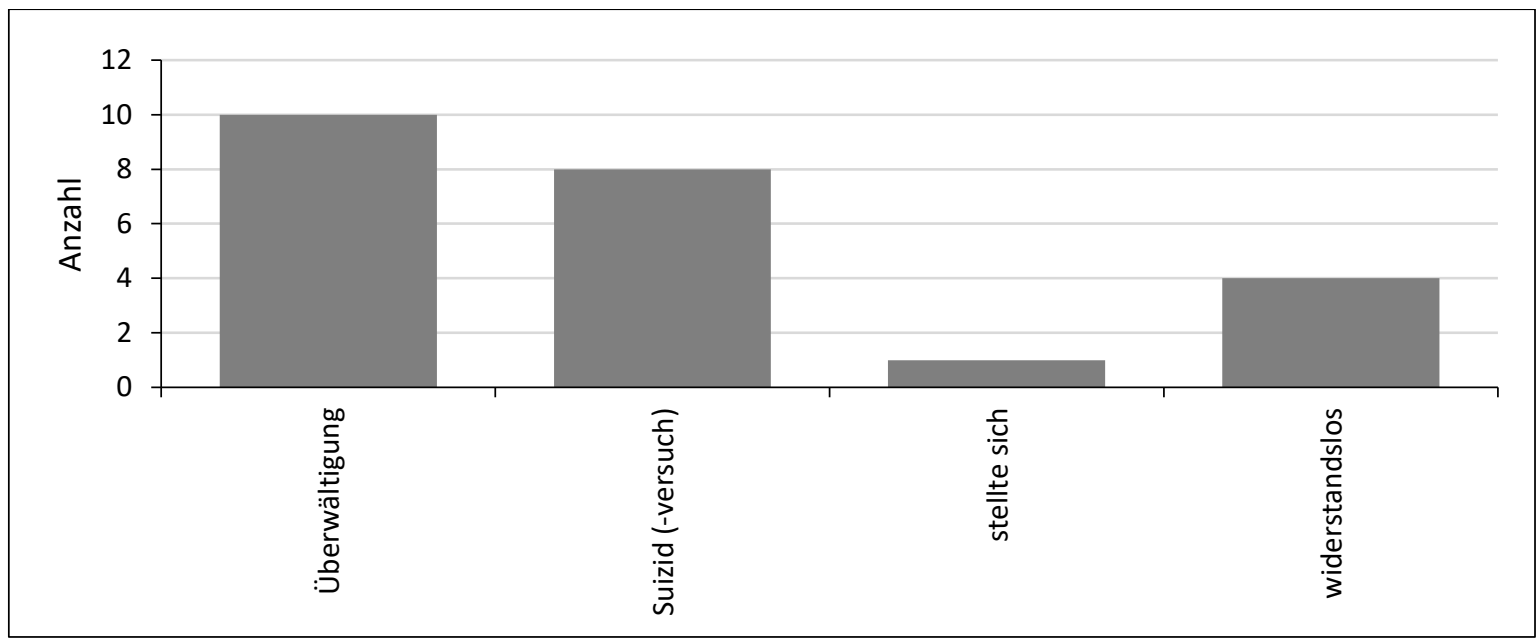

Abbildung 24: Ende Amokfahrt

Bei $13 \%$ der Fälle $(n=3)$ blieb unbekannt, wer nach Ende der Tat noch vor Ort bei dem Amokfahrer eine Diagnose stellte. Nur in einem Fall (4,3 \%) erfolgte die Diagnosestellung durch einen Arzt, bei $60,9 \%$ der Fälle $(n=14)$ erfolgte eine erste Diagnosestellung bzw. 
Einschätzung des Gesundheitszustandes des Amokfahrers durch Einsatzkräfte der Polizei vor Ort. In $21,7 \%$ der Fälle $(n=5)$ wurde anhand des geschilderten Tatverhaltens des Amokfahrers im Rahmen dieser Untersuchung eine Verdachtsdiagnose gestellt.

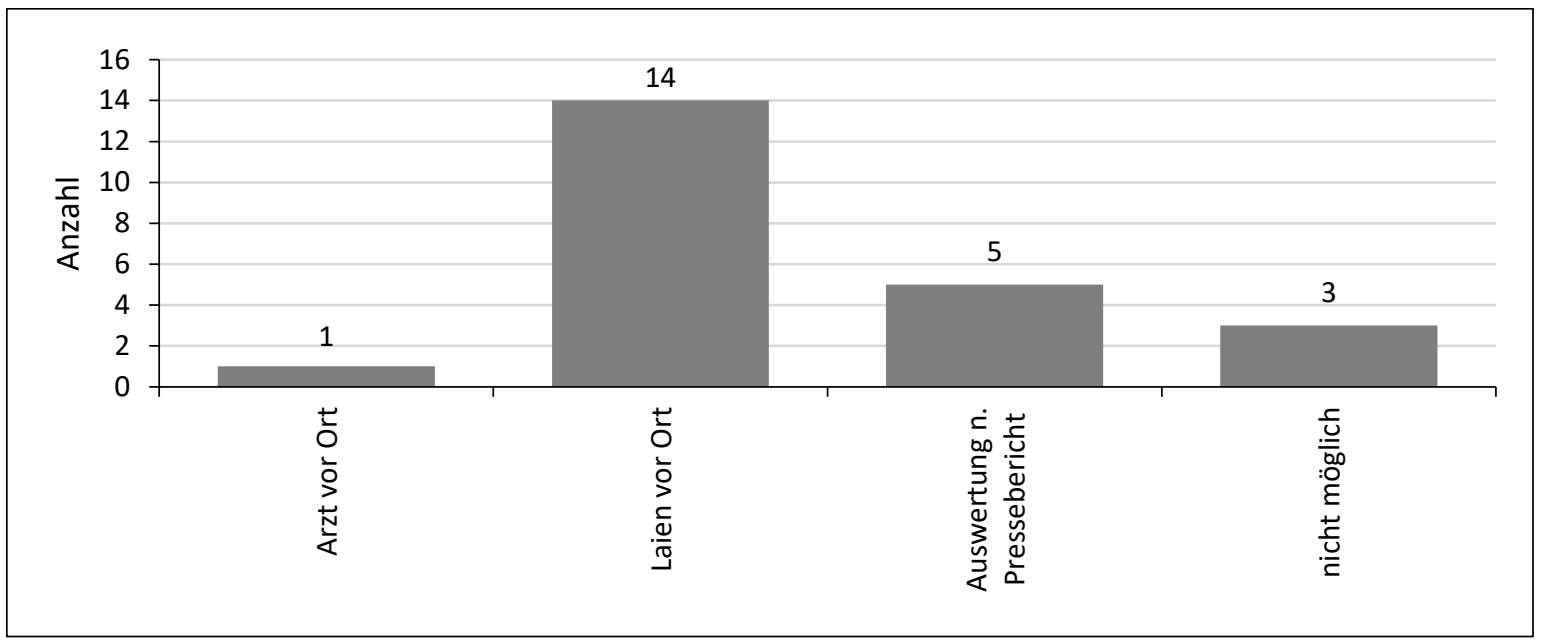

Abbildung 25: Art der Diagnosestellung

Die Diagnosestellung erfolgte zur Vergleichbarkeit der eigenen Daten mit denen der DreiDekaden-Amokstudie mit der Methode des sogenannten Deutschen Schichtenmodells. Gemeint ist hier das Schichten- oder Strukturmodell der menschlichen Psyche, das Sigmund Freud 1923 entwickelte und das als Deutsches Schichtenmodell in die psychoanalytische Literatur einging. Freud postulierte mit diesem Modell den Aufbau der menschlichen Psyche aus drei Schichten - dem Es, dem Ich und dem Über-Ich. Als Es bezeichnete Freud den unbewussten Teil der menschlichen Psyche, wo sich die Affekte und Impulse eines Menschen befinden, mit dem Ich das Ich-Bewusstsein und die Persönlichkeitsstruktur eines Menschen und mit dem Über-Ich die verinnerlichten Werte und Normen, nach denen eine Person ihr Handeln ausrichtet. Es lässt sich mit diesem Modell z. B. beschreiben, wie eine affektive Enthemmung infolge eines akuten psychischen Krankheits- oder Intoxikationszustandes bei entsprechender Intensität aus dem Unbewussten in das Bewusstsein eines Menschen einbricht und als emotional hoch aufgeladenes, jedoch nicht beabsichtigtes Verhalten nach außen dringt. Die affektive Enthemmung kann unter Umständen so stark sein, dass sie noch weiter in die Über-Ich-Schicht eindringt und dort verinnerlichte Regeln, Normen, Werte und Verbote außer Kraft setzt, so dass weder der Verstand (Ich-Schicht), noch moralische Überzeugungen (Über-Ich-Schicht) dem aus der Es-Schicht durchbrechenden Affektsturm standhalten können. Da sich mit diesem Modell auch das Phänomen Amok plausibel darstellen und nachvollziehen lässt, nutzte Adler das deutsche Schichtenmodell 
vielfach (Adler et al. 1993, 1994 und 2006), um einerseits das bei der Untersuchung des Amoktäters auffallende psychisch hoch auffällige Verhalten diagnostisch einzuordnen. Zum anderen wollte er nach der Beschreibung der Phänomenologie eines Amoktäters noch in tiefere Schichten seiner Persönlichkeit vorzudringen, um dort ggf. tiefer liegende Störungen festzustellen, die mit der hervorgetretenen Amoktat in Zusammenhang stehen bzw. ihr zugrunde liegen. Heute würde man stattdessen möglicherweise von weiteren bei einem Amoktäter vorliegenden Komorbiditäten sprechen, wobei der Begriff Komorbidität mit der inhaltlichen Bedeutung des Begriffs Schichtenmodell nicht übereinstimmt. Während Komorbidität mehrere nebeneinander und oft unabhängig voneinander bestehende Erkrankungen oder psychische Störungen bezeichnet, werden mit dem deutschen Schichtenmodell zwiebelschalenartig über- bzw. untereinander angeordnete psychische Erkrankungen oder Störungen bei einer Person beschrieben, von denen auf den ersten Blick nur die obersten Schicht zu erkennen ist.

Nach Diagnosestellung mit dem Deutschen Schichtenmodell lag bei 26,1 \% der Amokfahrer $(n=6)$ als offensichtliche Erkrankung eine schizophrene Psychose vor, bei weiteren $8,7 \%(\mathrm{n}=2)$ handelte es sich um eine unspezifische Psychose, bei zwei weiteren Amokfahrern $(8,7 \%)$ bestand eine Wahnkrankheit. Bei weiteren zwei Amokfahrern $(8,7 \%)$ lag eine schwere Persönlichkeitsstörung vor und bei sechs der Amokfahrer (26,1\%) stellte ihre Amokfahrt eine Affekthandlung nach einer akuten schweren Belastungssituation dar. Nur ein Amokfahrer dieser Untersuchung (4,3\%) war - nach allem was man weiß - psychisch gesund.

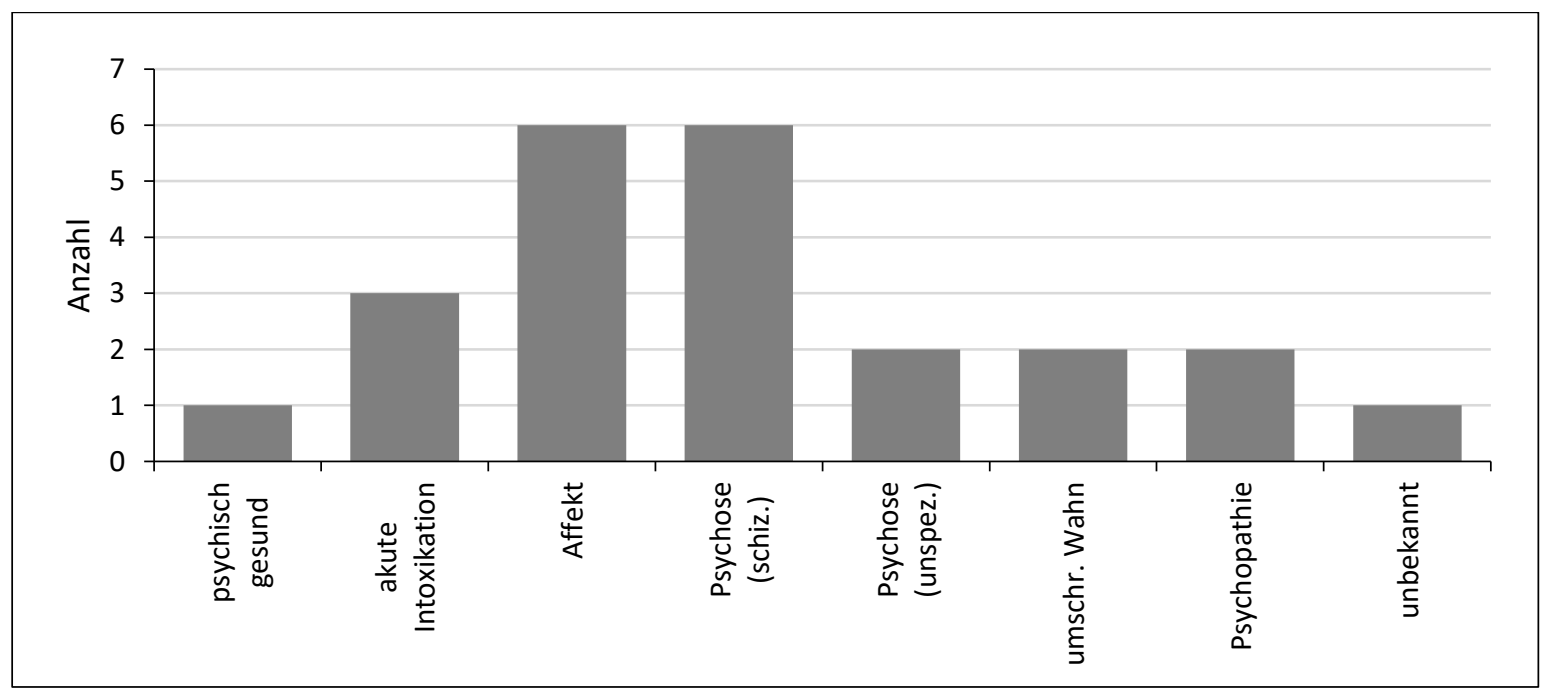

Abbildung 26: Diagnosestellung nach dem Deutschen Schichtenmodell 
Es waren 17,4 \% der Amokfahrer $(\mathrm{n}=4)$ während ihrer Tat Alkohol intoxikiert, $13 \%$ von ihnen $(\mathrm{n}=3)$ standen als Amokfahrer unter Einfluss von Amphetaminen. In 69,6 \% der Fälle $(\mathrm{n}=16)$ lagen keine Informationen über eine Intoxikation während der Amokfahrt vor.

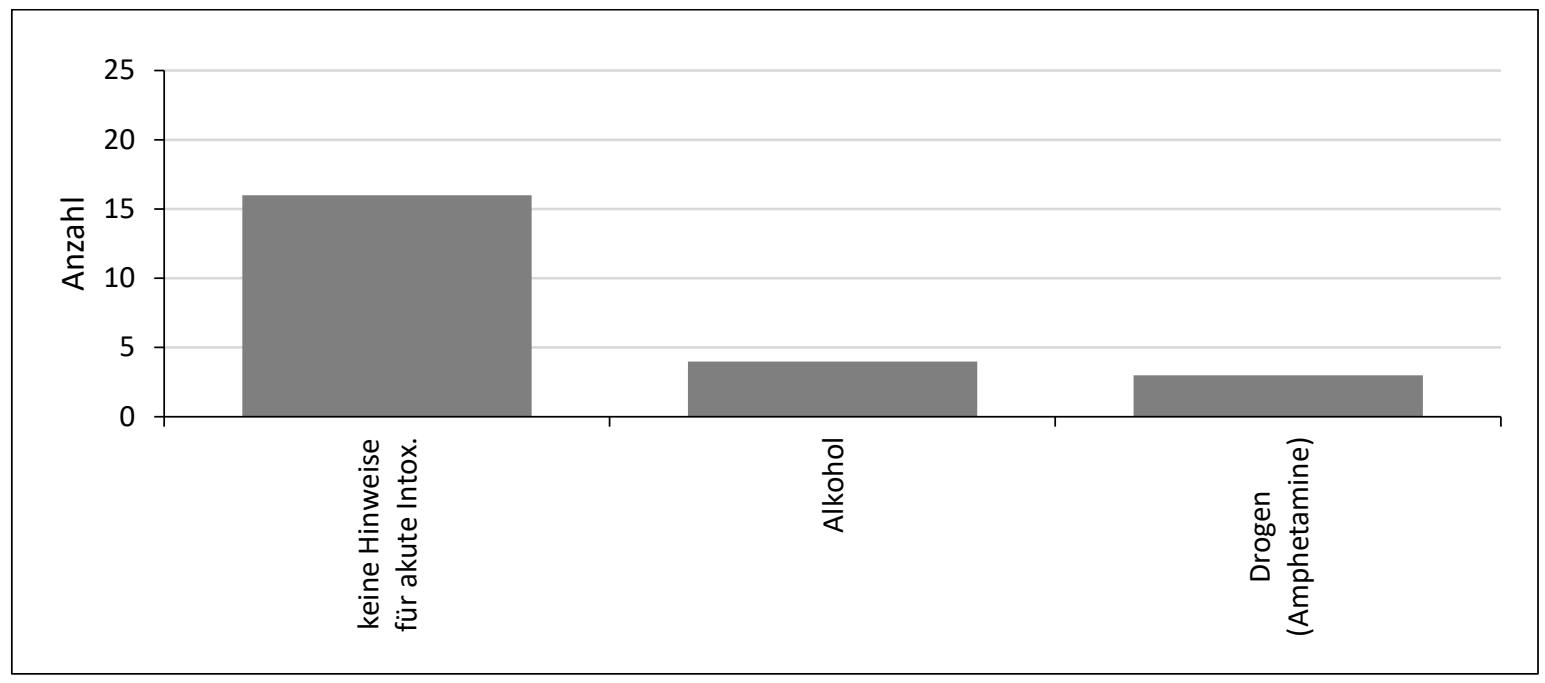

Abbildung 27: Akute Intoxikation

Bei 26,1 \% der Amokfahrer $(\mathrm{n}=6)$ bestand eine retrograde Amnesie für die Zeit ihrer Amokfahrt, zwei Amokfahrer (8,7 \%) schilderten starke Gefühle von Ärger, Wut oder Rache kurz vor und auch während ihrer Amokfahrt, acht Amokfahrer (34,8 \%) fielen nach der Tat durch verschiedene psychopathologische Syndrome auf, ein Amokfahrer (4,3 \%) konnte dazu keine Angaben machen, weil er seine Amokfahrt nicht überlebte und bei sechs Amokfahrern (26,1\%) lagen hierzu keine Informationen aus den Presseberichten vor. (Abb.: nächste Seite) 


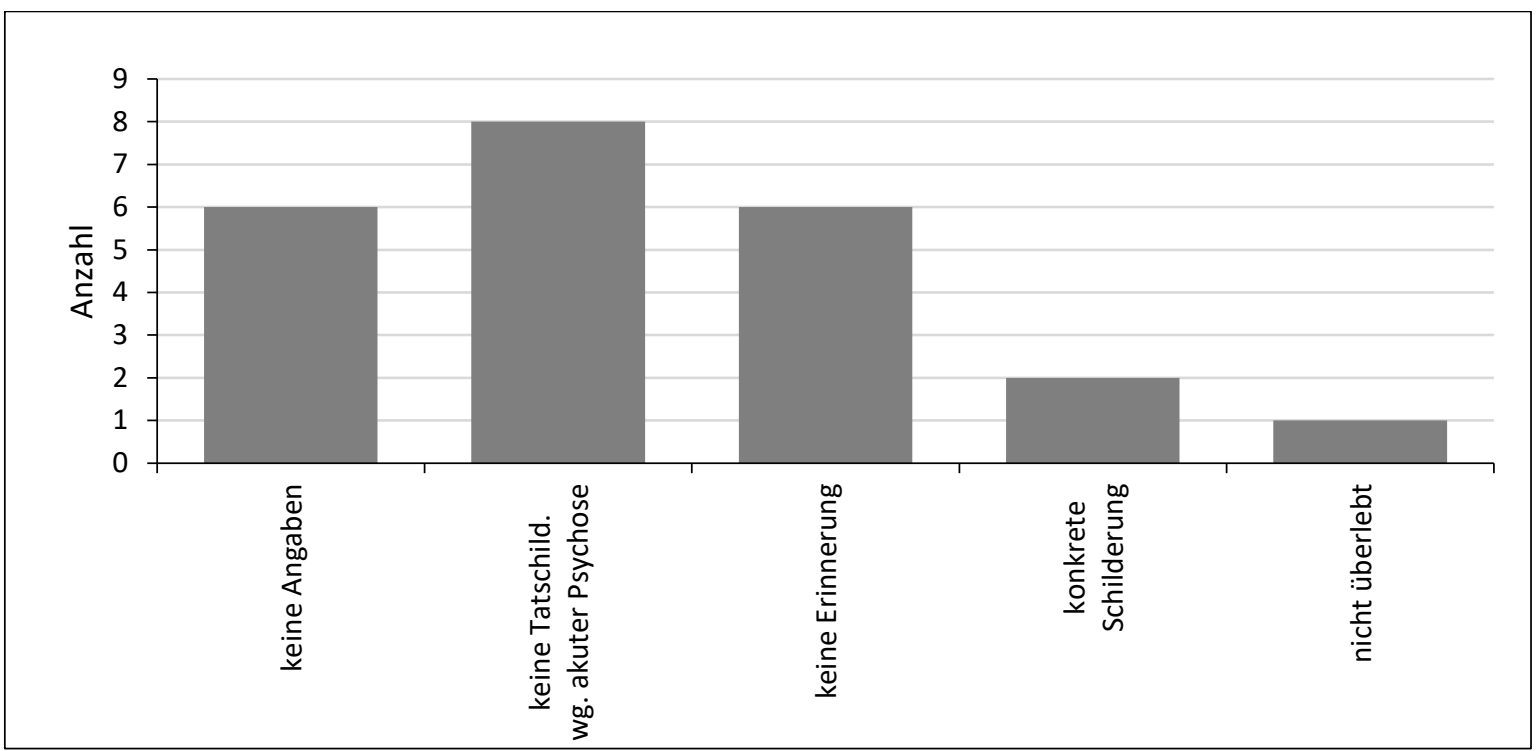

Abbildung 28: Angaben des Täters

Von den untersuchten Amokfahrern wurden 22 von 23 (95,7 \%) vor Gericht gestellt und rechtskräftig verurteilt. Ein Amokfahrer überlebte seine Amokfahrt nicht. Die Anklagepunkte bei ihnen lauteten gefährlicher Eingriff in den Straßenverkehr, Mord, versuchter Mord, Totschlag, gefährlicher Körperverletzung mit Todesfolge, Fahrerflucht und Widerstand gegen Vollstreckungsbeamte. Es wurden 39,1 \% der Täter $(n=9)$ zu Freiheitsstrafen verurteilt, davon zwei $(8,7 \%)$ zu einer Haftstrafe von zwei Jahren, einer $(4,3 \%)$ zu einer Haftstrafe von zwei Jahren und neun Monaten, ein weiterer (4,3 \%) zu einer Freiheitsstrafe von drei Jahren und einem Monat, ein Amokfahrer (4,3 \%) erhielt eine Haftstrafe von vier Jahren, ein weiterer $(4,3 \%)$ wurde zu einer Freiheitsstrafe von sechs Jahren verurteilt, ein Amokfahrer (4,3\%) zu eine Haftstrafe von sechs Jahren und sechs Monaten und ein weiterer $(4,3 \%)$ zu sieben Jahren Freiheitsentzug. Bei 14 Amokfahrern (60,9 \%) fanden sich keine Angaben über das weitere Täterschicksal.

\subsection{Amokfahrten in der Drei-Dekaden-Studie}

Die Untersuchungen über die Thüringer Amokfahrten 2001 - 2010 und die Untersuchungen über Amokfahrten deutschlandweit 2001 - 2010 gingen als kleiner, aber eigenständiger Teil in das anfangs schon erwähnte Drei-Dekaden-Amokprojekt von Adler (1993, 1994, 2006) 
ein. Während Schünemann (1991) unter den von ihm untersuchten 196 Amokfällen des Untersuchungszeitraums 1981 - 1990 (1. Dekade) 48 Amokfahrten fand (davon 31 in Deutschland), waren in der 2. Dekade (1991 - 2000) bei D. Marx (in Adler et al. 2006) nur zwei Fälle von Amokfahrten. Zusammen mit den eigenen 23 gesamtdeutschen Fällen der 3. Dekade

(2001 - 2010) waren es im gesamten Zeitraum der Amok-Langzeitstudie 73 Fälle von Amokfahrten, deren Auftreten sich im Gesamtverlauf diskontinuierlich darstellte

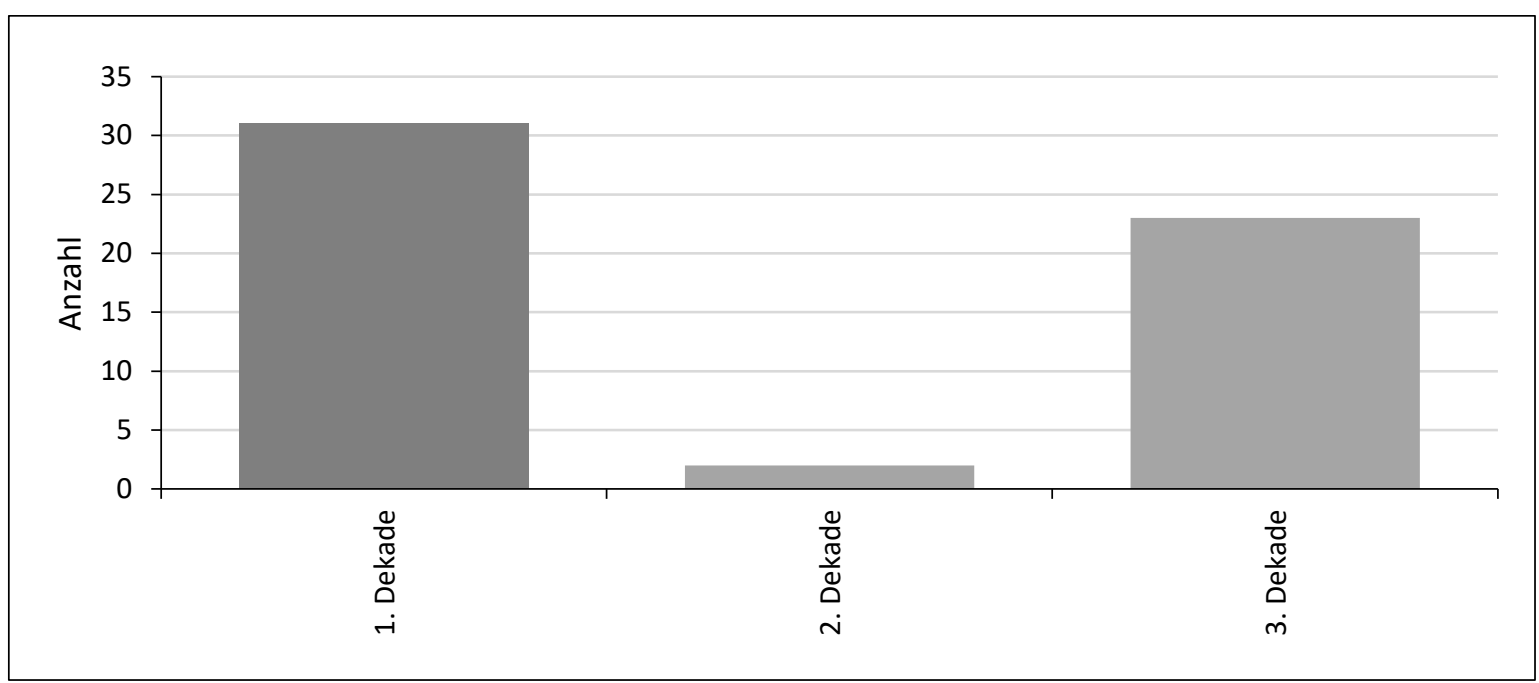

Abbildung 29: Anzahl der Amokfahrten deutschlandweit in den jeweiligen Dekaden 


\subsection{Berichte über Amokfahrten im europäischen Ausland im Zeitraum 2001 - 2010}

Im genannten Untersuchungszeitraum fanden sich in deutschen Presseberichten sowie in deutschsprachigen Online-Zeitungsausgaben aus Österreich und der Schweiz insgesamt 14 Artikel über Amokfahrten. Es handelte sich dabei um sechs Presseberichte über Amokfahrten aus Österreich, vier aus der Schweiz, zwei aus Frankreich. Je ein Bericht kam aus den Niederlanden und aus Schweden. Bei der Untersuchung dieser Berichte anhand der Amokkriterien zeigte sich, dass nicht alle Berichte tatsächliche Amokfahrten zum Inhalt hatten, sondern auch über andere schwere Verkehrsstraftaten berichteten. Es handelte sich dabei um: Aggressivität im Straßenverkehr (Tatmotiv: Rache): $n=3 ;(20 \%)$, psychisch auffällige (hier: akut psychotische) Autofahrer: $\mathrm{n}=12 ;(66 \%)$ und Amokfahrten: $\mathrm{n}=2$; $(11 \%)$

\subsection{Berichte in der deutschen Presse über außereuropäische Amokfahrten im Untersuchungszeitraum 2001 - 2010}

Auch nach intensiver Recherche fanden sich in der deutschen Presse des genannten Untersuchungszeitraums lediglich 10 Artikel, die über Fälle von Amokfahrten weltweit berichteten. vier Artikel berichteten über Amokfahrten, die sich in den USA ereigneten hatten, vier um Amokfahrten in Israel und je einer der Artikel berichtete über eine Amokfahrt in Mexiko bzw. China. Nach Prüfung aller 10 Fälle möglicher Amokfahrten anhand der Amokkriterien zeigte sich erneut, dass es sich nur bei einem kleinen Teil dieser Fälle um tatsächliche Amokfahrten handelte. Bei den vier angeblichen Amokfahrten in Israel handelte in jedem Fall um politisch motivierte Gewalttaten, bei denen Fahrzeuge als Tatwaffe eingesetzt wurden, um in terroristischer Absicht gezielt wehrlose Menschen zu töten. Alles in allem erbrachte die Untersuchung anhand der Amokkriterien in $40 \%$ der Fälle angeblicher Amokfahrten politisch motivierte Gewalt mit einem Fahrzeug als Waffe $(n=4)$, einen psychisch auffälligen Autofahrer (10\%), in drei Fällen Aggressivität im Straßenverkehr (30\%) und zwei echte Amokfahrten (20\%). 


\subsection{Zusammenfassung der Ergebnisse}

Bei der kontentanalytischen Auswertung mit Hilfe standardisierter Items (Kap. 6.1) gab es durchgängig in allen Presseartikeln ein Dunkelfeld an Informationen. Dieses Dunkelfeld entstand dadurch, dass die von der Untersucherin zum Zweck der Informationsgewinnung ausgewerteten Zeitungsartikel in keinem der Fälle zu allen 72 Items Informationen lieferten. Trotzdem gelang es, mit der kontentanalytischen Methode Informationen über Amokfahrer zu gewinnen, beginnend mit dem Alter der untersuchten Amokfahrer: Das Alter der Amokfahrer dieser Untersuchung streute und ohne erkennbaren Altersgipfel zwischen 19 und 79 Jahren mit einem Altersdurchschnitt von 35,5 Jahren. Von ihrer Lebenssituation vor der Amokfahrt wissen wir, dass 26,1 \% von ihnen allein lebten und 30,4\% in einer Partnerbeziehung. Es hatten 21,7 \% eine abgeschlossene Berufsausbildung als Arbeiter oder Handwerker (blue collar), $13 \%$ waren Angestellte (white collar) und weitere $13 \%$ besaßen einen Hochschulabschluss. Aber trotzdem fast die Hälfte der Amokfahrer über qualifizierte Abschlüsse verfügte, hatte nur 8,7 \% von ihnen einen sicheren Arbeitsplatz, weitere $8,7 \%$ lebten von Unterstützung und Gelegenheitsjobs - und 30,4 \% der späteren Amokfahrer im erwerbsfähigen Alter waren arbeitslos. Weitere 8,7 \% von ihnen, die ebenfalls nicht erwerbstätig waren, befanden sich bereits im Rentenalter.

Folgende Persönlichkeitsmerkmale kennzeichnen die Amokfahrer dieser Untersuchung: $17,4 \%$ von ihnen werden als impulsiv und unbeherrscht beschrieben, $13 \%$ waren schon im Vorfeld der Amokfahrt durch bedrohlichen Jähzorn aufgefallen. Weitere 8,7 \% zeigten querulatorische und hypochondrische, $8,7 \%$ paranoide bzw. anderweitig wahnhafte Auffälligkeiten und $30 \%$ der späteren Amokfahrer waren bereits im Vorfeld durch kriminelle Handlungen aus dem Bereich der einfachen Kriminalität (Körperverletzungsdelikte) aufgefallen. Von den Amokfahrern dieser Studie waren 95,7 \% männlichen Geschlechts, nur in einem einzigen Fall handelte es sich um eine Amokfahrerin. Von den untersuchten Amokfahrern waren 69,6 \% Deutsche, 26,6 \% stammten aus dem europäischen Ausland, lediglich ein Amokfahrer war außereuropäischer Herkunft.

Häufig traten im Vorfeld einer Amokfahrt bei dem späteren Täter schwerwiegenden lebensverändernde Ereignisse auf wie Ehescheidung/Partnerschaftstrennung (17,4 \%), schwerwiegende Lebenskonflikte (13\%), Arbeitsplatz- oder Wohnungsverlust (je ein Fall), Niederlage vor Gericht ein (Fall). Andererseits war bei 39,1 \% der untersuchten Amokfahrer war kein äußerliches Motiv für ihre Tat zu erkennen. Bei 52,2 \% der Amokfahrer war 
das jeweilige Tatmotiv unmittelbar handlungseinleitend, in einem Fall erfolgte die Tat sogar noch vor dem befürchteten Ereignis, das gleichzeitig das Tatmotiv darstellte. 21,7 \% der Amokfahrer wurden unmittelbar vor der Tat als aggressiv gespannt beschrieben, wobei der Tatbeginn bei diesen Amokfahrern unmittelbar erfolgte. Nur in einem Fall gab es eine Latenzzeit von mehreren Tagen, in einem anderen von mehreren Monaten zwischen dem tatauslösenden Motiv und der darauffolgenden Amokfahrt. Bei 21,7 \% der Amokfahrer war nach Zeugenangaben zu Beginn ihrer Tat keine äußere Affektspannung erkennbar, bei weiteren $21,7 \%$ war kein direkter Zusammenhang zwischen ihrem Vortatverhalten und ihrer anschließenden Amokfahrt erkennbar.

Die durchgeführte Auswertung der Amokfahrerfälle ergab außerdem, dass 52,2 \% der untersuchten Amokfahrer vor Begehung ihrer Tat psychisch erkrankt waren - vor allem an Psychosen aus dem schizophrenen Formenkreis. 20,4 \% dieser psychisch kranken Amokfahrer waren während ihrer Amokfahrt zusätzlich Alkohol- und/oder Drogen intoxikiert. Bei Letzterem handelte es sich zumeist um Intoxikationen mit Cannabis und/oder Amphetaminen. Bei 91,3 \% der untersuchten Amokfahrten gab es keine persönliche Täter-OpferBeziehung und 73,9 \% der Amokfahrer dieser Studie verletzten ihre Opfer wahllos. In $13 \%$ der Fälle fuhr der Amokfahrer mit seinem Tatfahrzeug direkt in eine Menschengruppe hinein und die Tat endete auch dort. Bei weiteren $13 \%$ der Amokfahrer begann die Amokfahrt ebenso, richtete sich aber in einem zweiten Teil der Tat wahllos gegen weitere Personen. Von allen hier untersuchten Amokfahrten dauerten mehr als die Hälfte (56,5 \%) nicht länger als eine Stunde. 69,6 \% der Amokfahrten begannen an einem umschriebenen Tatort, um sich dann im weiteren Tatverlauf (meist entlang der Straße, auf der die Amokfahrt stattfand) räumlich auszudehnen.

Fast die Hälfte der untersuchten Amokfahrer (47,8 \%) verursachte mit ihrer jeweiligen Amokfahrt extrem schwere Verkehrsunfälle mit zwischen einem und fünf Todesopfern. Rechnet man bei den Unfallopfern noch die Verletzten hinzu, so gab es bei $82,6 \%$ der untersuchten Amokfahrten zu zwischen einem und 26 Verletzten. Zusätzlich richteten wurden bei 78,3 \% der Amokfahrten erhebliche Sachschäden angerichtet. 82,6 \% der Amokfahrer flüchteten vom Unfallort und wurden erst nach einer längeren Verfolgungsfahrt durch die Polizei gestoppt, wobei 43,5 \% von ihnen erst aufgaben, nachdem sie von der Polizei gewaltsam gestoppt, überwältigt und entwaffnet worden waren. Alle Amokfahrer (bis auf einen, der am Ende seiner Amokfahrt Suizid beging) wurden vor Gericht gestellt und zu Freiheitsstrafen zwischen zwei und sieben Jahren verurteilt. Die beiden Thüringer 
Amokfahrer wurden wegen krankheitsbedingter Schuldunfähigkeit zum Zeitpunkt der Tat in einer psychiatrischen Klinik untergebracht.

\section{Diskussion}

\subsection{Soziodemographische Charakterisierung der Amokfahrer}

Ausgangspunkt dieser Untersuchung war, das Auftreten von Amokfahrten im Vergleich zu anderen Formen gefährlichen Fahrverhaltens im Straßenverkehr zu untersuchen und Aussagen zu treffen über die tatsächliche Häufigkeit des Auftretens von Amokfahrten. Aus methodologischer Sicht mag es nicht unproblematisch erscheinen, dass die Datengewinnung hierzu aus Presseberichten auf Basis von polizeilichen Unfallberichten erfolgte. Somit lag die primäre Deutungshoheit, ob es sich bei dem besonderen Verkehrsereignis um eine Amokfahrt gehandelt hat oder nicht, zuerst einmal bei dem Journalisten, der den Artikel schrieb. Und der erhielt seine Informationen über die Pressestelle der jeweiligen Polizeiinspektion, wo nach polizeiinternen Kriterien entschieden worden war, welche Informationen in welchem Umfang der Öffentlichkeit zugänglich gemacht werden sollen. Folge dieser doppelten Datenselektion ist ein Dunkelfeld bzw. eine nicht unerhebliche Missingdata-Struktur, die bei der eigenen Datenerhebung und Verschlüsselung der Daten in Items deutlich wurde. Aufgrund dieser Missing-data-Struktur konnten aus keinem der untersuchten Presseartikel Informationen zu allen 72 Items gewonnen werden, die später in die kontenanalytische Untersuchung eingingen. Dass die kontentanalytische Untersuchung von Presseartikeln eine anerkannte wissenschaftliche Methode zur Informationsgewinnung ist, wurde bereits unter Kap. 2.8 dargestellt. Im Folgenden sollen die Alleinstellungsmerkmale der Amokfahrt und des Amokfahrers im Vergleich zum Amoklauf und dem Amokläufer sowie den anderen schon genannten gefährlichen Verkehrsteilnehmern, die überdurchschnittlich viele schwere Verkehrsunfälle mit Personen- und Sachenschäden verursachen, diskutiert und dargestellt werden.

\subsection{Amokfahrer vs. Amokläufer}

Zur Tätertypologie des Amokfahrers konnten ausreichend Informationen gewonnen werden, um zeigen zu können, zwischen beiden Formen eines Amoktäters sowohl Gemein- 
samkeiten als auch Unterschiede gibt. Adler (2010a) beschreibt den Amokfahrer als Sonderform des Amokläufers. Beiden Tätertypen ist gemeinsam, dass mehr als 90 \% Männer mit einem durchschnittlichen Alter von 35,5 Jahren sind. Auch hinsichtlich ihrer beruflichen und Arbeitssituation gibt es kaum Unterschiede. Diese beginnen erst bei den primärpersönlichen Eigenschaften: Während der Amokläufer im Allgemeinen als gehemmtaggressiv und sozial isoliert beschrieben wird, finden sich beim Amokfahrer Eigenschaften wie Impulsivität und Unbeherrschtheit bis zum Jähzorn und nicht selten querulatorisches, hypochondrisches und paranoid-wahnnahes Verhalten. Auch die tatauslösenden Motive von Amokläufer und Amokfahrer sind verschieden: Während beim Amokläufer zumeist reale Trennungs-, Verlust- oder Konfliktsituationen das tatauslösende Motiv darstellen, sind es bei etwa $40 \%$ der Amokfahrer neben realem Verlust- oder Kränkungserleben wahnhafte, äußerlich nicht erkennbare Tatmotive. Eine Erklärung dafür könnte in der Täterpersönlichkeit liegen: Während es sich bei den Amokläufern überwiegend um Täter mit einer Persönlichkeit handelt, die geprägt ist durch ein hohes Maß an Kränkbarkeit, Rigidität, Depressivität und Rachephantasien, sind es bei den Amokfahrern nicht wenige Täter, die schon vor ihrer Amokfahrt an einer Psychose aus dem schizophrenen Formenkreis oder einem chronischen Wahn erkrankt waren.

Auch im Tatablauf unterscheiden sich Amoklauf und Amokfahrt: Während der klassische Amoklauf einen Vier-Phasen-Ablauf hat, beginnt die Amokfahrt in $90 \%$ der Fälle bereits unmittelbar nach dem tatauslösenden Ereignis. Ebenso unterscheiden sich Amoklauf und Amokfahrt bei der Opferwahl: Während der Amokläufer seine Tat zuerst gegen ihm bekannte Personen richtet und erst in der Phase der sekundären Tatausweitung auch ihm unbekannte Personen angreift, ist die Amokfahrt von vornherein wahllos und gegen Personen gerichtet, die dem Amokfahrer unbekannt sind. Der nächste Unterschied besteht in der Wahl der Tatwaffe: Da Amokläufer heutzutage fast immer Schusswaffen verwenden, gibt es beim Amoklauf regelhaft Todesopfer, aber kaum Sachschäden. Bei der Hälfte der Amokfahrten gibt es keine Todesopfer, aber mehr Verletzte und fast immer auch erhebliche Sachschäden. Auch das Ende der Tat ist verschieden: Während ganz im Sinne der Menninger-Trias die meisten Amokläufer ihre Tat nicht überleben, ist es bei den Amokfahrern genau umgekehrt. Mehr als 90 \% der Amokfahrer werden am Ende ihrer Amokfahrt von der Polizei festgenommen, vor Gericht gestellt und zu teil hohen Haftstrafen verurteilt (bzw. bei Schuldunfähigkeit in eine psychiatrische Klinik eingewiesen. 


\subsection{Amokfahrer in der 3-Dekaden-Studie in Deutschland}

Bis zu dieser Untersuchung stellten Amokfahrten im Rahmen der Langzeitstudie von Adler (2015) Zufallsbefunde dar, die anhand der Amokkriterien als Sonderform des Amoklaufs diagnostiziert, aber nicht weiter typisiert wurden. Während sich unter den von Schünemann (1991) untersuchten 196 Amokfällen der Dekade 1981 bis 199048 Amokfahrten befanden (31 davon aus Deutschland), waren es in der Dekade 1991 bis 2000 nur zwei (deutsche) Fälle, die im Rahmen einer Publikation von Adler et al. (2006) über Amokläufer als Zufallsbefunde auftraten. Die ausschließliche und gezielte Untersuchung von Amokfahrten für den Zeitraum der Dekade 2001 bis 2010 erfolgte im Rahmen dieser Studie, wobei für Thüringen lediglich zwei, für ganz Deutschland 23 gesicherte Fälle echter Amokfahrten (einschließlich der beiden Thüringer Fälle) aus Presseberichten verifiziert wurden. Mit einer solchen außerordentlichen Seltenheit des Vorkommens von Amokfahrten auf deutschen Straßen ist auch die scheinbare Diskontinuität zu erklären, mit der Amokfahrten von den drei Untersuchern der Drei-Dekaden-Studie gefunden wurden. Die relativ große Zahl von Amok-fahrten bei Schünemann (1991) könnte dabei ein Ausdruck des von Phillips (1977) beschriebenen „Nachahmer-Effekts“ sein: Bei den Amokfahrten in Schünemanns Untersuchung handelte sich in nicht geringer Zahl um spektakuläre Amokfahrten mit schweren militärischen Fahrzeugen wie Panzern u. ä. durch Soldaten der damals in Deutschland stationierten britischen bzw. amerikanischen Truppen. Diese Amokfahrten traten innerhalb eines relativ kurzen Zeitraums mehrfach hintereinander auf - und danach in dieser Häufigkeit bis heute nicht wieder. Eine weitere Erklärung für die Häufung von Amokfahrten in der ersten Dekade, ihrem fast vollständigen Rückgang in der zweiten Dekade und einer erneuten Zunahme in der dritten Dekade könnte mit der extremen Seltenheit des Vorkommens dieses Phänomens in Zusammenhang stehen: Auch wenn für die Untersuchungen bereits 10-Jahres-Zeiträume und ein Untersuchungsgebiet von der Größe der Bundesrepublik Deutschland gewählt wurden, konnten doch in der dritten Dekade nicht mehr als 23 echte Amokfahrten festgestellt werden. Das waren durchschnittlich 2,3 Amokfahrten pro Jahr, über den untersuchten 10-Jahres-Zeitraum hinweg jedoch diskontinuierlich auftraten.

Dass die Zahl der Amokfahrten in der zweiten Dekade im Vergleich zur ersten und dritten Dekade besonders niedrig ausfiel, lag auch am Untersuchungsgegenstand dieser Arbeit: Da sich die Untersucher hier in erster Linie mit Amokläufern befassten, waren die zwei zufäl- 
lig gefundenen Amokfahrten eher ein „Nebenprodukt“, das nicht in diese Untersuchungen einging. Eine andere Erklärung für das diskontinuierliche Auftreten der Amokfahrten könnte sein, dass die ausgewerteten Presseartikel immer nur einen zufälligen Ausschnitt des Auftretens von Amokfahrten erfassten. Um zu einem wesentlich größeren Datenpool an echten Amokfahrten zu kommen, müssten Untersuchungen über das Auftreten von Amokfahrten über noch deutlich längere Zeiträume als Dekaden durchgeführt werden - und/oder sich das Untersuchungsgebiet über eine wesentlich größere Fläche als Deutschland erstrecken. Solche Überlegungen sind denkbar und möglicherweise auch umsetzbar, würden aber den Rahmen einer Dissertationsarbeit sprengen.

\subsection{Abgrenzung von Amokfahrten gegenüber anderen Formen gefährlichen Fahr- verhaltens im Straßenverkehr}

Aufgrund der geringen Fallzahlen echter Amokfahrten in der Thüringen-Studie und der wenigen deutschen Presseberichte über Amokfahrer europa- und weltweit, erfolgte eine Falltypisierung, um tatsächliche Amokfahrten gegenüber anderen Formen gefährlichen Fahrverhaltens im Straßenverkehr eindeutig abzugrenzen. Schon bei der Untersuchung der Thüringer Amokfahrten (Kap. 2.2) mit Hilfe der Amokkriterien wurde deutlich, dass es sich bei den in der Thüringer Presse so genannten „Amokfahrten“ in 22,4 \% der Fälle in Wirklichkeit um eine erhebliche Verkehrsgefährdung durch Geister- oder Falschfahrer gehandelt hatte. In weiteren 19,7 \% der Fälle war es gefährliches Fahrverhalten psychisch auffälliger bzw. Alkohol- und/oder Drogen intoxikierter Autofahrer. In weiten 9,2 \% der Fälle von der Presse so genannter Amokfahrten waren es aggressive Autofahrer, die durch ihr Fahrverhalten andere Verkehrsteilnehmer erheblich gefährdeten. Dass bei der Untersuchung nur eine Suizidfahrt (1,32 \%) gefunden wurde, könnte die Vermutung von Petersen (1974) bestätigen, dass bei single car crashs mit tödlichem Ausgang die polizeilichen Ermittler am Unfallort das Vorliegen einer Suizidfahrt oft gar nicht in Erwägung ziehen. Andererseits könnte es aber auch ein Hinweis darauf sein, dass es mit der Presse eine Übereinkunft gibt, zur Verhinderung von Nachahmer-Suiziden über Suizidfälle im Allgemeinen und Suizidfahrten im Straßenverkehr im Besonderen nur in Ausnahmefällen zu berichten.

Neben den zwei gesicherten echten Amokfahrten (2,61\%) gab es in dieser Untersuchung noch weitere sechs nicht hinreichend gesicherte Fälle möglicher Amokfahrten $(6,5 \%)$ sowie anstelle tatsächlicher Amokfahrten andere Unfallursachen schwerer 
Verkehrsunfälle bzw. Verkehrsgefährdung wie Flucht vor der Polizei zur Verdeckung einer anderen Straftat, grober Unfug, jugendlicher Leichtsinn, akutes gesundheitliches Ereignis, die aber für die Falltypisierung letztlich keine Rolle spielten.

Bei den Presseartikeln über so genannte Amokfahrten aus dem europäischen Ausland handelte es sich neben zwei Fällen echter Amokfahrten (11\%) um gefährliches Fahrverhalten psychisch auffälliger (66 \%) oder aggressiver Autofahrer (20\%). Die deutschen Presseartikel über Amokfahrten weltweit berichteten über zwei Fälle echter Amokfahrten, ansonsten aber über Fälle von politisch motivierter Gewalt, bei denen die Täter schwere motorisierte Fahrzeuge in Menschgruppen lenkten, um zu töten und zu verletzen (40 \%) bzw. um gefährliches Fahrverhalten aggressiver Autofahrer (30 \%). Es wurde dabei deutlich, dass ungeachtet des jeweiligen Untersuchungsgebietes (Bundesland Thüringen, Deutschland, Europa, weltweit) echte Amokfahrten sehr selten vorkommen. Von der Presse häufig „Amokfahrer“ genannte andere besonders unfallträchtige Autofahrertypen zeigen in ihrem Verhalten teilweise Ähnlichkeiten mit dem echten Amokfahrer, können aber mit Hilfe der Amokkriterien eindeutig von diesem abgegrenzt werden.

So gibt es Übereinstimmungen zwischen dem aggressiven Autofahrer und dem Amokfahrer: Beide sind in ihrer überwiegenden Mehrheit Männer, haben häufig einen impulsivunbeherrschten Charakter und sind oft schon vorher anderweitig kriminell in Erscheinung getreten (Whitlock 1971, Michalowski 1975, Grey et al. 1989, Greiner 1995). Unterschiede finden sich zuerst einmal beim Alter: Mit einem Durchschnittsalter von 21,5 Jahren sind die aggressiven Autofahrer (Adelt et al. 1999) deutlich jünger als die Amokfahrer mit einem Durchschnittsalter von 35,5 Jahren. Der Hauptunterschied zwischen beiden Fahrergruppen ist jedoch die Intention ihres Handelns: Während der Amokfahrer sein Auto intentional als Waffe gegen andere Verkehrsteilnehmer einsetzt, ist beim aggressiven Autofahrer eine solche Intentionalität nicht vorhanden. Durch ihr rücksichtslos-egoistisches und instrumentell-aggressives Fahrverhalten, mit dem sie im öffentlichen Straßenverkehr in Erscheinung treten (Maag und Krüger 2003), handeln sie nicht absichtsvoll in der Art des Amokfahrers, sondern gefährden auf verantwortungslose Weise andere Verkehrsteilnehmer wie auch ihr eigenes Leben. Aggressive Autofahrer nehmen es billigend in Kauf, dass sie durch ihre unfallträchtige Fahrweise mit hoher Wahrscheinlichkeit zum Verursacher schwerer Verkehrsunfälle werden können, dabei andere Verkehrsteilnehmer auf extrem fahrlässige Weise verletzen oder gar töten oder bei dem von ihnen verursachten Unfall auch selbst zu Tode kommen können. Das spektakulär-gefährliche und unfallträchtige Fahrverhalten 
dieser jungen, aggressiven männlichen Autofahrer wird, so zeigte diese Untersuchung, von der Presse nicht selten medienwirksam als „Amokfahrt“ bezeichnet und machte bei der Thüringer Untersuchung 9,2 \% aller so genannter Amokfahrten aus. Es handelte sich hierbei aber schon deshalb nicht um echte Amokfahrten, weil bei ihnen das Kennzeichen der Intentionalität der Tat nicht vorlag.

Übereinstimmungen wurden auch zwischen Amokfahrern und akut psychisch kranken bzw. alkohol- und/oder Drogen intoxikierten Autofahrern gefunden: Aus der Gruppe der psychisch kranken bzw. psychisch gestörten Autofahrer verursachen Personen mit schweren Persönlichkeitsstörungen am häufigsten schwere Unfälle mit Personenschäden (Kastrup et al. 1978). Fong et al. (2001) verwiesen bei den Unfallverursachern besonders auf die Gruppe der Autofahrer mit einer aggressiv-feindseligen Persönlichkeit. Die zweitgrößte Gruppe besonders unfallträchtiger Autofahrer stellen nach Kastrup et al. (1978) alkoholund/oder Drogen intoxikierte Autofahrer dar. Wenn jedoch ein Autofahrer mit einer aggressiv-feindseligen Persönlichkeit noch zusätzlich alkohol- und/oder Drogen intoxikiert im Straßenverkehr unterwegs ist, so erhöht sich sein Risiko, einen schweren Verkehrsunfall mit Personenschäden zu verursachen, noch einmal deutlich (Nabi et al. 2006). Hier gibt es zusätzlich auch noch eine Querverbindung zwischen psychisch auffälligem und aggressivem Autofahrer: Mehrere Autoren (Mayer 1987, Fong et al. 2001) sind der Meinung, dass bei Autofahrern, die alkoholisiert oder Drogen intoxikiert am Steuer sitzen und bei denen gleichzeitig Persönlichkeitseigenschaften wie Feindseligkeit und ein hohes Maß an Kränkbarkeit vorliegen, ein besonders hohes Risiko besteht, schwere Verkehrsunfälle mit Personenschäden zu verursachen. Während in den Untersuchungen von Kastrup et al. (1978) über psychisch kranke und gestörte Autofahrer Personen mit schweren Persönlichkeitsstörungen mit 22,2 \% die größte Gruppe der Unfallverursacher bildeten, lagen an zweiter Stelle die alkohol- und drogenkranken Unfallverursacher (15,8\%), gefolgt von Personen nach einer akuten Belastungsreaktion (13,2 \%) und von Personen mit einer affektiven oder psychotischen Erkrankung (10,4 \%). Weitere 38,4 \% der Fälle dieser Untersuchung verteilten sich auf eine größere Anzahl von Diagnosen im einstelligen Bereich, die aber für diese Untersuchung nicht von Bedeutung sind. Im Unterschied dazu zeigte sich bei den Amokfahrern der eigenen Untersuchung eine ganz andere Diagnoseverteilung: Bei 52,2 \% aller Amokfahrer bestand zum Zeitpunkt ihrer Amokfahrt eine akute Psychose aus dem schizophrenen Formenkreis, bei 40,1 \% von ihnen lag eine schwere Persönlichkeitsstörung vor und 20,4 \% waren zum Zeitpunkt ihrer Amokfahrt alkohol- und/oder Drogen intoxikiert. 
Die Suchtmittelintoxikation bestand nur in einigen Fällen ausschließlich $(7,7 \%)$, bei 12.7 \% der intoxikierten Amokfahrer lag eine Kombination der Diagnosen schwere Persönlichkeitsstörung und akute Alkoholintoxikation bzw. akute Psychose und akute Drogenintoxikation vor. Eine sichere Unterscheidung zwischen psychisch auffälligen u./o. intoxikierten Unfallfahrern und echten Amokfahrern ist auch in diesem Fall nur durch die Prüfung der Intentionalität des Geschehens zu erreichen: Der psychisch gestörte oder suchtkranke Autofahrer ist aufgrund der Auswirkungen seiner schweren akuten psychischen Erkrankung, seiner schweren Persönlichkeitsstörung oder seiner akuten Suchtmittelintoxikation nicht oder nicht ausreichend in der Lage ist, sein Fahrzeug im Straßenverkehr verkehrssicher zu führen und die Verkehrsregeln ausreichend sorgfältig zu beachten, um keinen Verkehrsunfall zu verursachen. Diese erheblich unfallträchtigen Autofahrer handeln, wenn sie sich ungeachtet ihrer aktuell verminderten Fahrtüchtigkeit hinter das Steuer setzen, grob fahrlässig, aber nicht intentional. Sie legen es nicht von vornherein darauf an, mit ihrer zustandsbedingt gefährlichen Fahrweise anderen Verkehrsteilnehmern Schaden zuzufügen, nehmen aber mit ihrer Entscheidung, sich in ihrem nicht oder nicht ausreichend fahrtüchtigen Zustand mit dem Auto in den öffentlichen Verkehr zu begeben, billigend in Kauf, dass sie eine Gefahr für andere Verkehrsteilnehmer sind. Allerdings verursacht diese Personengruppe weitaus häufiger schwere Verkehrsunfälle mit Personenschäden als die Gruppe der echten Amokfahrer. Wegen der Schwere der von ihnen verursachten Verkehrsunfälle und des oft spektakulären Unfallgeschehen wird in der Presse in solchen Fällen oft von Amokfahrten gesprochen, ohne dass es sich tatsächlich um solche handelt. Ein Amokfahrer handelt per definitionem immer intentional. Oft selbst zum Zeitpunkt seiner Amokfahrt in einem psychischen Ausnahmezustand, in einigen Fällen noch zusätzlich Drogen intoxikiert, ist seine Tat, nicht selten motiviert durch sein Wahnerleben, von Anfang an darauf ausgerichtet, ihm unbekannten Verkehrsteilnehmern größtmöglichen Schaden zuzufügen und dabei seinen eigenen Tod durch einen Verkehrsunfall zu suchen oder billigend in Kauf zu nehmen (was wieder eine Schnittmenge mit den Suizidfahrern darstellt).

Das heißt, dass es zwischen Amokfahrern und Suizidfahrern Übereinstimmungen gibt aber auch eindeutige Unterschiede. Die von Ford und Moseley (1963), MacDonald (1964), Hamburger (1969) untersuchten Suizidfahrer waren alle im Vorfeld ihrer Autosuizids manifest psychisch krank (und einige von ihnen noch zusätzlich alkoholkrank), was wiederum eine Schnittstelle mit der Gruppe der psychisch kranken Unfallfahrer darstellt. Eine Ge- 
meinsamkeit von Amokfahrern und Suizidfahrern ist, dass ihr gefährliches Verhalten im Straßenverkehr darauf gerichtet ist zu töten. Beide Fahrertypen handeln intentional, gerichtet auf ein Ergebnis ihrer Fahrt, dass sie erreichen wollen. Ein wesentlicher Unterschied zwischen Amokfahrer und Suizidfahrer ist die Ausrichtung ihres intentionalen Handelns: Ein Suizidfahrer ist intentional ausschließlich darauf ausgerichtet, sich selbst durch einen ebenfalls von ihm selbst intendierten „Verkehrsunfall" (häufig als Fahrt mit einem Pkw mit hoher Geschwindigkeit, nicht angeschnallt und ungebremst gegen einen Baum, einen Betonpfeiler oder als absichtlicher Frontalzusammenstoß mit einem wesentlich größeren Fahrzeug) zu töten (Schmidt et al. 1972). Der Amokfahrer ist zwar intentional ebenfalls darauf gerichtet, sich durch seine Amokfahrt zu töten oder dabei getötet zu werden (zweites und drittes Menninger-Kriterium), ist aber in seiner Intentionalität primär darauf gerichtet, mit seinem Fahrzeug als Waffe andere Personen zu töten, zu verletzen und größtmögliche Schäden anzurichten. Während ein Suizidfahrer, wenn er im Straßenverkehr absichtlich entgegen der Richtungsfahrbahn unterwegs ist (hier bildet der Suizidfahrer eine Schnittmenge mit dem Falschfahrer) einen Frontalzusammenstoß mit einem wesentlich größeren Fahrzeug (z. B. Lkw) anstrebt, um sich dadurch sicher zu töten (nicht aber den Fahrer des anderen Fahrzeugs), fährt der Amokfahrer als Falschfahrer (hier besteht eine Schnittmenge zwischen Amokfahrer und Falschfahrer) intentional und gerichtet frontal auf ein anderes Fahrzeug ähnlicher Größe mit hoher Geschwindigkeit zu in der Absicht, größtmögliche Schäden (insbesondere Personenschäden) anzurichten und die Tat auch selbst nicht zu überleben (Homizid-Suizid-Tat). Somit unterscheidet sich die Amokfahrt als Homizid-Suizid-Tat eindeutig von der Suizidfahrt als reiner Suizid-Tat.

Amokfahrer können, wie soeben dargestellt, bei ihrer Amokfahrt auch als Falschfahrer im Straßenverkehr unterwegs sein. Sie können dann vor dem Fahrer des Fahrzeugs, dem sie mit hoher Geschwindigkeit (in einem Fall im Dunkeln ohne Licht) entgegenfahren, im wahrsten Sinne des Wortes wie ein Geisterfahrer plötzlich auftauchen und so ihn und mögliche Insassen seines Fahrzeugt in akute Lebensgefahr bringen. Einigen der Autofahrer, denen plötzlich ein Amokfahrer in der Art eines Geisterfahrers entgegenkam, gelang es noch im letzten Moment, dem Amokfahrer auszuweichen und sich zu retten, indem die Fahrbahn mit der so genannten „Flucht ins Grüne“ nach rechts verließen und dadurch leichtere Schäden und Verletzungen davontrugen. Wo das nicht gelang, kam es zu einem katastrophalen Unfall mit Toten, Verletzten und erheblichen Sachschäden. Es zeigen sich 
hier Gemeinsamkeiten zwischen Geisterfahrer und Amokfahrer, da beide mit ihrer Fahrt entgegen der Richtungsfahrbahn unterwegs sind und damit eine erhebliche Gefahr für alle anderen Autofahrer darstellen, die entsprechend des Richtungsfahrgebots rechts fahren. Während sich der bewusste Falschfahrer (Färber und Färber 1980) dazu entschied, kurzzeitig entgegen der Fahrtrichtung zu fahren (z. B. um auf diese Weise einen vorherigen Fahrfehler zu korrigieren), ist seine Intention jedoch darauf gerichtet, seine Fahrt so bald als möglich in der richtigen Richtung fortzusetzen. Er geht bei seinem Fahrmanöver bewusst ein erhebliches Risiko ein und handelt damit im verkehrsrechtlichen Sinne grob fahrlässig. Der unbewusste Falschfahrer (Färber und Färber 1980) ist aus fehlender Übersicht oder aus einer Überforderungssituation heraus auf die Gegenfahrbahn geraten und wurde so unabsichtlich zum Geisterfahrer. Bei ihm ist keinerlei Intentionalität zur Begehung seiner Falschfahrt erkennbar. Er wurde zum Geisterfahrer entweder infolge einer Fehlentscheidung bei fehlender Übersicht über die Verkehrssituation (bei dichtem Nebel fälschlich statt der Autobahnauffahrt die Autobahnabfahrt zum Auffahren gewählt, das falsche Verkehrsleitzeichen bei unübersichtlicher Ausschilderung gewählt) oder infolge akuter Fahruntüchtigkeit (unterwegs im Straßenverkehr als alkohol- und/oder Drogen intoxikierter Autofahrer) - oder infolge Uneinsichtigkeit bei bereits eingetretener dauerhafter Fahruntüchtigkeit beim hochbetagten Autofahrer mit bereits fortgeschrittener Demenz. Weder der bewusste Falschfahrer noch der unabsichtlich zum Geisterfahrer gewordene Autofahrer handeln intentional in der Absicht zu töten wie der Amokfahrer. Aber aus den verschiedenen genannten Gründen können sie, während sie im Straßenverkehr entgegen der Richtungsfahrbahn im Straßenverkehr unterwegs sind, zu einer erheblichen Gefahr für andere Verkehrsteilnehmer werden und schwere Unfälle mit Personen- und Sachschäden verursachen.

\subsection{Ausblick}

Die Ergebnisse dieser Untersuchung bestätigten die verwendete Arbeitshypothese, nach der Amokfahrten im öffentlichen Straßenverkehr extrem seltene Ereignisse sind. Der möglicherweise durch Presseveröffentlichungen entstandene Eindruck, dass Amokfahrten im Straßenverkehr häufiger vorkommen als früher, konnte widerlegt werden. Weiterhin wurde gezeigt, dass es außer den Amokfahrern noch weitere Autofahrertypen gibt, die überdurch- 
schnittlich häufig schwere Verkehrsunfälle verursachen. Diese haben Gemeinsamkeiten mit dem Amokfahrer, unterscheiden sich aber gleichzeitig von ihm eindeutig Es konnte gezeigt werden, dass sich unter den Amokfahrern dieser Untersuchung überdurchschnittlich viele psychisch kranke Personen befanden, bei denen es infolge eines ungünstigen Zusammentreffens von subjektiven und objektiven Belastungsfaktoren zu einem Impulskontrollverlust mit der Folge einer sich unmittelbar daran anschließenden Amokfahrt kam. Es könnte Forschungsgegenstand einer weiterführenden Arbeit sein, valide Parameter zu entwickeln, mit denen die Wahrscheinlichkeit des Auftretens einer Impulstat (wie sie auch die Amokfahrt darstellt) bei gefährdeten Personen prognostisch beurteilt werden könnte.

\section{Zusammenfassung}

Ziel dieser Arbeit war es, die Häufigkeit des Vorkommens von Amokfahrten im Straßenverkehr zu untersuchen. Mit Hilfe der wissenschaftlichen Amokkriterien war es möglich, echte Amokfahrten von anderen, zumindest äußerlich homizidal-suizidal erscheinenden, gefährlichen und erheblich unfallträchtigem anderen Phänomenen im Straßenverkehr eindeutig abzugrenzen und Aussagen über die tatsächliche Häufigkeit des Auftretens von Amokfahrten zu treffen. Es konnte gezeigt werden, dass Amokfahrten im Spektrum schwerer Verkehrsunfälle mit Personenschäden nur einen verschwindend geringen Anteil ausmachen. Es konnte weiterhin gezeigt werden, dass nur die allerwenigsten der spektakulären Verkehrsstraftaten, die von den Verfassern der untersuchten Presseartikel mit „Amokfahrten“ betitelt wurden, die Kriterien einer echten Amokfahrt erfüllten. Des Weiteren konnte gezeigt werden, dass bei den Amokfahrern psychische Störungen (und hierbei vor allem akute Psychosen und schwere Persönlichkeitsstörungen) im Vergleich zur Allgemeinbevölkerung deutlich häufiger anzutreffen sind. Beide Thüringer Amokfahrer waren während ihrer Amokfahrten akut psychotisch, bei beiden war bereits vor der Tat eine Psychose aus dem schizophrenen Formenkreis diagnostiziert worden. An einer Psychose leiden ca. 5 \% an der Gesamtbevölkerung Deutschlands, Millionen Menschen sind täglich Autofahrer im Straßenverkehr unterwegs. Dabei ist nicht auszuschließen, dass darunter Menschen sind, die an einer Psychose erkrankt sind. Würde allein das Vorliegen einer Psychose bei einem Autofahrer dazu führen, dass er zum Amokfahrer wird, müsste somit die Zahl der Amokfahrten auf bundesdeutschen Straßen deutlich höher liegen, als es diese Untersuchung 
zeigen konnte. Das zeigt, dass psychische Erkrankung oder schwere Persönlichkeitsstörung nicht die alleinigen Voraussetzungen darstellen, um zum Amokfahrer zu werden. Die Erklärung dafür ist, dass für eine Amokfahrt mehrere tatauslösende Faktoren zeitnah zusammentreffen müssen. $\mathrm{Zu}$ diesen Faktoren zählen: Ein überwältigendes Lebensereignis, das krankheits- oder störungsbedingt paranoid verarbeitet wird, eine erhöhte psychische Vulnerabilität und ein unmittelbarer Zugang zu einem motorisiertem Fahrtzeug als Tat waffe. Der psychische Ausnahmezustand, in dem sich eine Person unter den geschilderten Umständen befindet, führt dann zu dem für Amok typischen Kontrollverlust, der unmittelbar zur Tatausführung, d. h. zum Beginn der Amokfahrt überleitet, wobei das tatauslösende Ereignis von außen gesehen eher banal und geringfügig erscheint. Das im Gegensatz dazu unverhältnismäßig erscheinende Kränkungserleben des späteren Amokfahrers ist nur nachvollziehbar, wenn man seinen psychosenahen oder bereits psychotischen inneren Zustand in die Beurteilung einbezieht. So wird die äußere Situation entsprechend des inneren Erlebens umgedeutet. Der Impetus eines Amokfahrers bei Beginn und Durchführung seiner Amokfahrt steht somit in einem direkten Zusammenhang mit den von der akuten psychotischen Symptomatik in seinem Inneren ausgehenden heftigen Gewaltimpulsen. Dass er über diese Gewaltimpulse während der Amokfahrt zunehmend die Kontrolle verliert und nur noch äußere Gewalt wie Polizeimaßnahmen gestoppt werden kann, zeigte die Beispiele echter Amokfahrten in dieser Arbeit. Ist es möglich, eine Amokfahrt zu verhindern, indem man den Täter zuvor schon einer Behandlung zuführt? Es gibt in der Bevölkerung durchaus etliche Personen, die chronisch psychisch krank sind und in krisenhaften Lebenssituationen deutlich weniger Ressourcen haben, auf angemessene Weise damit umzugehen. Viele von ihnen haben sich aus persönlichen und psychosebedingten Gründen noch nie freiwillig in fachärztliche Behandlung gegeben. Kann die Gefahr, die von solchen Personen, die zum Amokfahrer werden könnten, verringert werden - und wenn ja, wie? Grundsätzlich ist es unerlässlich, dass die Grunderkrankung einer solchen Person behandelt wird, weil, wie wir sahen, Wahndynamik und gewalttätige Wahnimpulse die Triebkräfte einer Amokfahrt darstellen. Wenn sich aber eine psychisch kranke und verhaltensauffällige Person nicht aus eigenem Antrieb in psychiatrische Behandlung begibt, sondern stattdessen externalisierend und auf aggressive Weise für seine Probleme Menschen in ihrem Umfeld verantwortlich macht und diese zum Teil angreift, wäre es wichtig, dass öffentliche Dienste wie der Sozialpsychiatrische Dienst einbezogen werden. Wegschauen und sich nicht zu- 
ständig fühlen lässt die Probleme einer Person wie im Falle des Holger Z. weiter ,hochkochen“ und kann, wie wir gesehen haben, in eine Amokfahrt münden. 


\section{Anhang}

Tabellen A1 (Items) und A2 (Auflistung der ausgewerteten Presseartikel)

$\mathrm{Zu}$ Tabelle A1: Items

Das kontentanalytische Verfahren zur Auswertung von Amokfällen wurde in dieser Untersuchung angewandt auf Amokfahrten der Dekade III des gesamten Bundesgebiets (Kap. 2.3). Diese wurden im Sinne einer Re-Analyse mit den bereits erhobenen entsprechenden und bereits ausgewerteten Fällen der Dekaden I und II in Beziehung gesetzt. Die zu vergleichenden Fälle aller drei Dekaden wurden mit demselben 72-Item-Schema ausgewertet, um so eine Vergleichbarkeit der Daten untereinander zu erreichen.

Zu Tabelle A2: Presseartikel

Diese Tabelle liegt zur besseren Lesbarkeit im Querformat dar. 


\begin{tabular}{|c|c|c|}
\hline Item Nr. & Beschreibung & Bewertung \\
\hline $1-2$ & Fall Nummer & (Zehner, Einer) \\
\hline 3 & Typ-Code Nr. & $\begin{array}{l}0=\text { unbekannt } \\
1=\text { Schulamok } \\
\mathbf{2}=\text { Autoamok (Verkehr) } \\
3=\text { Behörde } \\
4=\text { offene Plätze } \\
5=\text { sonstiges }\end{array}$ \\
\hline $4-9$ & $\begin{array}{l}\text { Datum der Tat oder ggf. nur } \\
\text { Meldung }\end{array}$ & $\begin{array}{l}\text { (Tag, Monat und Jahr, falls Tag nicht bekannt }= \\
\text { xx) }\end{array}$ \\
\hline 10 & Geschlecht & $\begin{array}{l}0=\text { unbekannt } \\
1=\text { männlich } \\
2=\text { weiblich }\end{array}$ \\
\hline $10-11$ & Alter & (zweistellig: Jahre, $\mathrm{x}=$ unbekannt) \\
\hline 13 & Familienstand & $\begin{array}{l}0=\text { unbekannt } \\
1=\text { ledig immer Single } \\
2=\text { verheiratet } \\
3=\text { in Trennung lebend } \\
4=\text { getrennt lebend } \\
5=\text { verwitwet } \\
6=\text { geschieden } \\
7=\text { andere Lebensgemeinschaft } \\
8=\text { wiederverheiratet } 0 . \text { neue Partnerschaft }\end{array}$ \\
\hline 14 & $\begin{array}{l}\text { Lebensgemeinschaft } \\
\text { aktuell }\end{array}$ & $\begin{aligned} 0= & \text { unbekannt } \\
1= & \text { altersinadäquat bei den Eltern }- \\
& \text { nicht Kinder oder Jugendliche, diese dann } \underline{4} \\
2= & \text { Ehefrau } \\
3= & \text { Familie, d.h. Ehefrau und Kind/-er } \\
4= & \text { anderer Partner o. Gruppe, alters- } \\
& \text { angemessen bei den Eltern } \\
5= & \text { Heim oder andere geschützte Umgebung } \\
6= & \text { allein }\end{aligned}$ \\
\hline $\begin{array}{l}15 \\
\text { (Forts. } \\
\text { nächste } \\
\text { Seite }\end{array}$ & $\begin{array}{l}\text { Level der Berufsausbildung/- } \\
\text { ausübung }\end{array}$ & $\begin{aligned} 0= & \text { unbekannt } \\
1= & \text { ungelernt } \\
2= & \text { unstete Versuche, die keine Zuordnung er } \\
& \text { möglichen } \\
3= & \text { Handwerker o. vergleichbare Berufe (blue } \\
& \text { collar) o. entsprechende alterstypische Aus } \\
& \text { bildung dazu }\end{aligned}$ \\
\hline
\end{tabular}




\begin{tabular}{|c|c|c|}
\hline Item Nr. & Beschreibung & Bewertung \\
\hline & & $\begin{aligned}= & \text { Angestellter oder vergleichbare Berufe } \\
& \text { (white collar) o. entsprechende alterstyp- } \\
& \text { ische Ausbildung dazu } \\
5= & \text { Akademiker, leitende Position o. entsprech- } \\
& \text { ende alterstypische Ausbildung dazu }\end{aligned}$ \\
\hline 16 & $\begin{array}{l}\text { Aktuelles Beschäftigungs- } \\
\text { verhältnis }\end{array}$ & $\begin{array}{l}0=\text { unbekannt } \\
1=\text { arbeitslos } \\
2=\text { Job } \\
3=\text { feste Anstellung, Schüler, Student } \\
4=\text { Rentner }\end{array}$ \\
\hline 17 & Nationalität & $\begin{aligned} 0= & \text { unbekannt } \\
1= & \text { Deutscher } \\
2= & \text { sonstiger Europäer } \\
3= & \text { nichteuropäischer Mittelmeerraum (Türken, } \\
& \text { Marokkaner, Tunesier usw.) } \\
4= & \text { Nordamerika } \\
5= & \text { sonstige }\end{aligned}$ \\
\hline 18 & $\begin{array}{l}\text { Militärische o. paramilitäri- } \\
\text { sche Ausbildung }\end{array}$ & $\begin{array}{l}0=\text { unbekannt } \\
1=\text { keine } \\
2=\text { Soldat } \\
3=\text { Polizei } \\
4=\text { Schützenverein } \\
5=\text { Wachdienst }\end{array}$ \\
\hline 19 & $\begin{array}{l}\text { Persönlichkeitsauffälligkeiten } \\
\text { generell erwähnt }\end{array}$ & $\begin{array}{l}0=\text { unbekannt } \\
1=\text { zutreffendes Merkmal } \\
\mathbf{2}=\text { ausdrücklich nicht }\end{array}$ \\
\hline 20 & keine Auffälligkeiten erwähnt & $\begin{array}{l}0=\text { unbekannt } \\
1=\text { zutreffendes Merkmal } \\
\mathbf{2}=\text { ausdrücklich nicht }\end{array}$ \\
\hline 21 & $\begin{array}{l}\text { kontaktscheu, passiv, zu- } \\
\text { rückgezogen }\end{array}$ & $\begin{array}{l}0=\text { unbekannt } \\
1=\text { zutreffendes Merkmal } \\
\mathbf{2}=\text { ausdrücklich nicht }\end{array}$ \\
\hline 22 & $\begin{array}{l}\text { sehr aktiv, sich in den Vor- } \\
\text { dergrund stellend (jedoch } \\
\text { dabei nicht körperlich ag- } \\
\text { gressiv und jähzornig - das } \\
\text { gehört zu Item 30) }\end{array}$ & $\begin{array}{l}0=\text { unbekannt } \\
1=\text { zutreffendes Merkmal } \\
\mathbf{2}=\text { ausdrücklich nicht }\end{array}$ \\
\hline 23 & $\begin{array}{l}\text { immer sexuell abstinent, kei- } \\
\text { ne Beziehungen zu Ge- } \\
\text { schlechtspartnern bekannt }\end{array}$ & $\begin{array}{l}0=\text { unbekannt } \\
1=\text { zutreffendes Merkmal } \\
\mathbf{2}=\text { ausdrücklich nicht }\end{array}$ \\
\hline
\end{tabular}




\begin{tabular}{|c|c|c|}
\hline Item $\mathrm{Nr}$. & Beschreibung & Bewertung \\
\hline 24 & $\begin{array}{l}\text { ungewöhnliche sexuelle Ver- } \\
\text { haltensweisen o. Perversion } \\
\text { bekannt } 0 \text {. vermutet }\end{array}$ & $\begin{array}{l}0=\text { unbekannt } \\
1=\text { zutreffendes Merkmal } \\
\mathbf{2}=\text { ausdrücklich nicht }\end{array}$ \\
\hline 25 & Waffennarr, -sammler & $\begin{array}{l}0=\text { unbekannt } \\
1=\text { zutreffendes Merkmal } \\
\mathbf{2}=\text { ausdrücklich nicht }\end{array}$ \\
\hline 26 & $\begin{array}{l}\text { schießt aktuell im Beruf o. } \\
\text { im Verein }\end{array}$ & $\begin{array}{l}0=\text { unbekannt } \\
1=\text { zutreffendes Merkmal } \\
\mathbf{2}=\text { ausdrücklich nicht }\end{array}$ \\
\hline 27 & $\begin{array}{l}\text { hat bereits außerhalb sozial } \\
\text { üblicher Situationen - zum } \\
\text { Spaß o. in Konfliktsituatio- } \\
\text { nen - "herumgeballert" }\end{array}$ & $\begin{array}{l}0=\text { unbekannt } \\
1=\text { zutreffendes Merkmal } \\
\mathbf{2}=\text { ausdrücklich nicht }\end{array}$ \\
\hline 28 & $\begin{array}{l}\text { querulatorische, hypochond- } \\
\text { rische, paranoide o.a. wahn- } \\
\text { kranke Auffälligkeiten }\end{array}$ & $\begin{array}{l}0=\text { unbekannt } \\
1=\text { zutreffendes Merkmal } \\
\mathbf{2}=\text { ausdrücklich nicht }\end{array}$ \\
\hline 29 & $\begin{array}{l}\text { impulsiv und unbeherrscht } \\
\text { (o. Körperverletzung, gehört } \\
\text { in Item 30) }\end{array}$ & $\begin{array}{l}0=\text { unbekannt } \\
1=\text { zutreffendes Merkmal } \\
\mathbf{2}=\text { ausdrücklich nicht }\end{array}$ \\
\hline 30 & $\begin{array}{l}\text { bedrohlicher Jähzorn mit } \\
\text { tätlicher Fremdaggressivität } \\
\text { (Körperverletzung) }\end{array}$ & $\begin{array}{l}0=\text { unbekannt } \\
1=\text { zutreffendes Merkmal } \\
\mathbf{2}=\text { ausdrücklich nicht }\end{array}$ \\
\hline 31 & $\begin{array}{l}\text { resignativ-depressive Ver- } \\
\text { stimmung, Minderwertig- } \\
\text { keitsgefühle }\end{array}$ & $\begin{array}{l}0=\text { unbekannt } \\
1=\text { zutreffendes Merkmal } \\
\mathbf{2}=\text { ausdrücklich nicht }\end{array}$ \\
\hline 32 & Suizidversuch & $\begin{array}{l}0=\text { unbekannt } \\
1=\text { zutreffendes Merkmal } \\
\mathbf{2}=\text { ausdrücklich nicht }\end{array}$ \\
\hline 33 & $\begin{array}{l}\text { kriminelle Handlungen im } \\
\text { Vorfeld, Vorstrafen }\end{array}$ & $\begin{array}{l}0=\text { unbekannt } \\
1=\text { zutreffendes Merkmal } \\
\mathbf{2}=\text { ausdrücklich nicht }\end{array}$ \\
\hline 34 & $\begin{array}{l}\text { auffällige Wesensänderung } \\
\text { im Vorfeld der Tat }\end{array}$ & $\begin{array}{l}0=\text { unbekannt } \\
1=\text { zutreffendes Merkmal } \\
\mathbf{2}=\text { ausdrücklich nicht }\end{array}$ \\
\hline 35 & $\begin{array}{l}\text { psychiatrische Erkrankung o. } \\
\text { Störung vor der Tat bekannt? }\end{array}$ & $\begin{array}{l}0=\text { unbekannt } \\
1=\text { zutreffendes Merkmal }\end{array}$ \\
\hline
\end{tabular}




\begin{tabular}{|c|c|c|}
\hline Item Nr. & Beschreibung & Bewertung \\
\hline & & $\mathbf{2}=$ ausdrücklich nicht \\
\hline 36 & $\begin{array}{l}\text { militärische Spezialausbil- } \\
\text { dung (Mitglied einer Spezial- } \\
\text { einheit, Einzelkämpfer, Teil- } \\
\text { nahme an Kriegseinsätzen) }\end{array}$ & $\begin{array}{l}0=\text { unbekannt } \\
1=\text { zutreffendes Merkmal } \\
\mathbf{2}=\text { ausdrücklich nicht }\end{array}$ \\
\hline $37-39$ & $\begin{array}{l}\text { Tatmotive (bis zu } 3 \text { Tatmoti- } \\
\text { ve können angegeben wer- } \\
\text { den, das schwerwiegendste } \\
\text { zuerst und wie folgt ver- } \\
\text { schlüsselt): }\end{array}$ & $\begin{aligned} 0= & \text { unbekannt } \\
1= & \text { kein nachvollziehbares Tatmotiv belegt o. } \\
& \text { Bagatellauslöser } \\
2= & \text { Scheidung, Trennung u. damit in Zusam- } \\
& \text { menhang stehende Eifersucht } \\
3= & \text { andere Objektverluste (Tod des Partners, } \\
& \text { eines Elternteils, eines Kindes u. ä. } \\
4= & \text { Partnerschaftskonflikte, andere private } \\
& \text { Konflikte } \\
5= & \text { Strafmandat, -prozess, andere Geldstrafen, } \\
& \text { Konflikte mit öffentlichen Organen } \\
6= & \text { Entlassung, ungewollte Umsetzung, Kon- } \\
& \text { flikte am Arbeitsplatz, Arbeitslosigkeit, } \\
& \text { Rentenkampf } \\
7= & \text { fehlende Wohnung, extreme Schulden, ext } \\
& \text { reme materielle Not } \\
8= & \text { rassistische Motive o. Ähnliches, } \\
9= & \text { pseudo-rassistische/-politische Gründe, in } \\
& \text { Wirklichkeit wahnhaft }\end{aligned}$ \\
\hline 40 & $\begin{array}{l}\text { äußere Affektladung vor der } \\
\text { Tat (z.B. als Folge der letzten } \\
\text { tatauslösenden Belastung wie } \\
\text { ein Strafmandat o.ä.) }\end{array}$ & $\begin{aligned} 0= & \text { unbekannt } \\
1= & \text { aggressive Auseinandersetzung } \\
2= & \text { äußerlich keine aggressive Auseinanderset } \\
& \text { zung erkennbar } \\
3= & \text { nicht zutreffend, kein unmittelbarer motiva- } \\
& \text { tionaler Zusammenhang }\end{aligned}$ \\
\hline 41 & äußeres Verhalten vor der Tat & $\begin{array}{l}0=\text { unbekannt } \\
1=\text { aggressiv, erregt } \\
2=\text { äußerlich abnorm ruhig und gefasst }\end{array}$ \\
\hline 42 & $\begin{array}{l}\text { Zeit - Tat - Motivbeziehung } \\
\text { für das wichtigste Motiv } \\
\text { nach menschlichem Ermes- } \\
\text { sen (nicht: "der letzte Trop- } \\
\text { fen") }\end{array}$ & $\begin{aligned} 0= & \text { unbekannt } \\
1= & \text { sofort handlungseinleitend } \\
2= & \text { nach einer Latenzzeit von } 1-30 \text { Tagen } \\
3= & \text { nach einer Latenzzeit von } 1-12 \text { Monaten } \\
4= & \text { nach einer Latenzzeit von mehr als } 1 \mathrm{Jahr} \\
5= & \text { vor kränkenden/bedrohlichen Ereignissen } \\
& \text { wie drohender Kündigung, Frau droht, ihn } \\
& \text { zu verlassen, reicht die Scheidung ein, Frau } \\
& \text { schwerkrank, droht zu sterben } \mathrm{u} . \text { ä. m. }\end{aligned}$ \\
\hline
\end{tabular}




\begin{tabular}{|c|c|c|}
\hline Item Nr. & Beschreibung & Bewertung \\
\hline $43-44$ & Anzahl der Toten & (zweistellig, $\mathrm{x}=$ unbekannt) \\
\hline $45-46$ & Anzahl der Verletzten & (zweistellig, $\mathrm{x}=$ unbekannt) \\
\hline 47 & Opfergeschlecht & $\begin{array}{l}0=\text { unbekannt } \\
1=\text { überwiegend männlich } \\
2=\text { überwiegend weiblich } \\
3=\text { wahllos gemischt }\end{array}$ \\
\hline 48 & Opferalter & $\begin{array}{l}0=\text { unbekannt } \\
1=\text { überwiegend Kinder und Jugendliche } \\
2=\text { überwiegend Erwachsene } \\
3=\text { wahllos gemischt }\end{array}$ \\
\hline 49 & Sachschaden & $\begin{aligned} 0= & \text { unbekannt } \\
1= & \text { erheblicher Sachschaden, der das zufällige } \\
& \text { Ausmaß deutlich überschreitet wie Zerstö- } \\
& \text { rung ganzer Reihen von Pkw o. eines o. } \\
& \text { mehrerer Häuser } \\
2= & \text { kein o. nur geringer Sachschaden, gewis- } \\
& \text { sermaßen unvermeidlich wie Zerstörung ei } \\
& \text { ner Fensterscheibe beim Schießen o. Total } \\
& \text { schaden am eigenen Auto }\end{aligned}$ \\
\hline 50 & $\begin{array}{l}\text { Konflikt-/problembezogene } \\
\text { Täter-Opfer-Beziehung }\end{array}$ & $\begin{aligned} 0= & \text { unbekannt } \\
1= & \text { begrenzt auf eine umschriebene Gruppe wie } \\
& \text { Konfliktpartner o. Familie, Handlung durch } \\
& \text { Dritte begrenzt } \\
2= & \text { beginnend mit umschriebener Gruppe, ver- } \\
& \text { sehentliche Mitbeteiligung zufällig an- } \\
& \text { wesender Dritter } \\
3= & \text { beginnend mit umschriebener Gruppe, akti- } \\
& \text { ve und wahllose Ausdehnung auf Andere } \\
4= & \text { wahllos von vornherein }\end{aligned}$ \\
\hline 51 & $\begin{array}{l}\text { Persönliche Täter-Opfer- } \\
\text { Beziehung }\end{array}$ & $\begin{array}{l}0=\text { unbekannt } \\
1=\text { nur eigene Kinder } \\
2=\text { nur Ehefrau/Partnerin } \\
3=\text { nur eigene Familie einschl. der Kinder } \\
4=\text { wie } 3 \text { und weitere Angehörige } \\
5=\text { nur Freunde und Bekannte } \\
6=\text { wie } 4 \text { und zusätzlich Fremde } \\
7=\text { wie } 6 \text { zusätzlich Bekannte } \\
8=\text { nur völlig Fremde }\end{array}$ \\
\hline $52-53$ & Tatbeginn & $\begin{array}{l}\text { (Uhrzeit zweistellig, grobe Angaben wie mit- } \\
\text { tags o.ä. wird als } 12 \text { Uhr codiert, Uhrzeit wird } \\
\text { auf volle Stundenzahl aufgerundet, }\end{array}$ \\
\hline
\end{tabular}




\begin{tabular}{|c|c|c|}
\hline Item Nr. & Beschreibung & Bewertung \\
\hline & & Uhrzeit unbekannt $=\mathrm{x}$ ) \\
\hline $54-55$ & Tatdauer & (in Stunden, zweistellig, keine Angabe $=\mathrm{x}$ ) \\
\hline 56 & Waffenbeschaffung & $\begin{array}{l}0=\text { unbekannt } \\
1=\text { zufällig greifbare Waffe o.ä. } \\
2=\text { schon vorbereitete Waffe(n) } \\
3=\text { Waffe immer verfügbar, z.B. Auto }\end{array}$ \\
\hline 57 & Art der Waffe & $\begin{aligned} 0= & \text { unbekannt } \\
1= & \text { Schusswaffe } \\
2= & \text { andere übliche Waffe, wie Messer, } \\
& \text { Hammer, Beil, Schwert u. ä. } \\
3= & \text { Waffenarsenal, d.h. mehr als eine Schuss- } \\
& \text { waffe, zusätzlich andere Waffen wie } \\
& \text { Brandbomben u.a. } \\
4= & \text { atypische Gegenstände, die an sich keine } \\
& \text { Waffen sind, z.B. verschiedenartige Fahr- } \\
& \text { zeuge, u.a. Autos } \\
5= & \text { ungewöhnliche entfremdete/umgebaute } \\
& \text { Gegenstände) }\end{aligned}$ \\
\hline 58 & Wechsel des Tatorts & $\begin{aligned} 0= & \text { unbekannt } \\
1= & \text { Tat beginnt und endet an einem um- } \\
& \text { schriebenen Tatort wie Schule, Arbeitsplatz } \\
& \text { u. ä. } \\
2= & \text { Tat wird räumlich über ein größeres Areal } \\
& \text { ausgedehnt und fortgesetzt }\end{aligned}$ \\
\hline 59 & Ausgang für den Täter & $\begin{aligned} 0= & \text { unbekannt } \\
1= & \text { Suizid o. Suizidversuch, der nur durch } \\
& \text { Zufall überlebt wurde } \\
2= & \text { Suizidversuch ,„mit weichen Methoden“ } \\
3= & \text { Tötung durch die Polizei oder Dritte } \\
4= & \text { gewaltsame Entwaffnung nach Verletzung, } \\
& \text { Widerstand bis zum Schluss } \\
5= & \text { widerstandslose Festnahme o. nur sehr } \\
& \text { geringer Widerstand } \\
6= & \text { Selbststellung, passives Verharren am Platz }\end{aligned}$ \\
\hline 60 & $\begin{array}{l}\text { Flucht des Täters ( = Verlas- } \\
\text { sen des Tatortes/-raumes } \\
\text { nach Beendigung der Tat, } \\
\text { auch Rückzug zum final an- } \\
\text { gelegten Suizid) }\end{array}$ & $\begin{aligned} 0 & =\text { unbekannt } \\
1 & =\text { ja, Motiv unklar } \\
2 & =\text { ja, Motiv klar, } z . B . \text { Rückzug zum finalen } \\
& \quad \text { Suizid } \\
3 & =\text { ja }, \text { echter Fluchtversuch }\end{aligned}$ \\
\hline
\end{tabular}




\begin{tabular}{|c|c|c|}
\hline Item Nr. & Beschreibung & Bewertung \\
\hline & & $4=$ nein \\
\hline 61 & Art der Diagnosestellung & $\begin{aligned} 0= & \text { unbekannt } \\
1= & \text { Diagnose-/Verdachtsdiagnose-Stellung } \\
& \text { durch Psychiater/Psychologen vor Ort, } \\
2= & \text { Verdachtsdiagnose durch Untersucher } \\
& \text { anhand von Täter-Symptomen, nicht } \\
& \text { Tathergang, } \\
3= & \text { Diagnosestellung durch medizinische Laien } \\
& \text { vor Ort wie Polizei, Journalisten } u \text {. ä.) }\end{aligned}$ \\
\hline 62 & $\begin{array}{l}\text { Diagnosestellung nach dem } \\
\text { Deutschen Schichtenmodell }\end{array}$ & $\begin{aligned} 0= & \text { unbekannt } \\
1= & \text { schizophrene Psychose und V. a. sch. Ps., } \\
& \text { mindestens 1 Erstrangs-Symptom wie } \\
& \text { akustische Halluzinationen müssen vor- } \\
& \text { handen sein, ggf. auch gleichzeitig } \\
& \text {,Verschrobenheit“ } \\
2= & \text { unspezifische Psychose mit mind. Irgend- } \\
& \text { einer Halluzination, groben Verhaltens- } \\
& \text { störungen wie Desorientiertheit } \\
3= & \text { Wahnkrankheit, z.B. fixierte Paranoia, } \\
& \text { Hy pochondrie, querulatorischer Wahn u. ä. } \\
& \text { Störungen } \\
4= & \text { Manie/Depressionen phasisch, über das } \\
& \text { Maß einer reaktiven Verstimmung hinaus } \\
& \text { gehend } \\
5= & \text { Psychopathie, d.h. andauernde Verhaltens- } \\
& \text { abweichungen des Denkens und des emoti- } \\
& \text { onalen Reagierens } \\
6= & \text { Affekthandlung nach umschriebener } \\
& \text { schwerwiegender Belastung bei ansonsten } \\
& \text { vorher unauffälliger Persönlichkeit } \\
7= & \text { soziokulturell bedingt abweichendes } \\
& \text { Verhalten, z.B. Rache wegen Ehrverlust } \\
8= & \text { akute Intoxikation o. V. a., z.B. bei } \\
& \text { Alkoholkranken } \\
9= & \text { gesund, nach allem, was man weiß) }\end{aligned}$ \\
\hline 63 & $\begin{array}{l}\text { Akute Intoxikation, hervor- } \\
\text { gerufen durch: }\end{array}$ & $\begin{aligned} 0= & \text { unbekannt } \\
1= & \text { Alkohol (Alkoholspiegel im Blut mehr als } \\
& 1 \text { Promille) } \\
2= & \text { halluzinogene Drogen wie z.B. Haschisch } \\
& \text { oder LSD }\end{aligned}$ \\
\hline
\end{tabular}




\begin{tabular}{|c|c|c|}
\hline Item Nr. & Beschreibung & Bewertung \\
\hline & & $\begin{aligned} 3= & \text { Stimulantien wie z.B. Kokain und } \\
& \text { Amphetamine) }\end{aligned}$ \\
\hline 64 & Tatort & $\begin{array}{l}0=\text { unbekannt } \\
1=\text { ländliche Gegend } \\
2=\text { Kleinstadt unter } 40.000 \text { Einwohner } \\
3=\text { Großstadt } \\
4=\text { Weltstadt mit mehr als } 1 \text { Mio. Einwohner }\end{array}$ \\
\hline 65 & $\begin{array}{l}\text { Tatort, bezogen auf eine Re- } \\
\text { gion in Deutschland }\end{array}$ & $\begin{aligned} 0= & \text { unbekannt } \\
1= & \text { Norddeutschland mit Niedersachsen, } \\
& \text { Schleswig-Holstein, Hamburg und Bremen } \\
2= & \text { Mitteldeutschland mit Hessen, NRW, } \\
& \text { Rheinland-Pfalz und dem Saarland } \\
3= & \text { Süddeutschland mit Baden-Württemberg } \\
& \text { und Bayern } \\
4= & \text { neue Bundesländer mit Mecklenburg- } \\
& \text { Vorpommern, Brandenburg mit Berlin, } \\
& \text { Sachsen-Anhalt, Sachsen und Thüringen }\end{aligned}$ \\
\hline 66 & SummFalltyp & $\begin{aligned} 0= & \text { unbekannt } \\
1= & \text { klassisch, Tötung im Umfeld, geht auf die } \\
& \text { Straße } \\
2= & \text { Familie } \\
\mathbf{3}= & \text { Auto, aber nicht einfache Geisterfahrt } \\
4= & \text { atypischer Amok }\end{aligned}$ \\
\hline 67 & Täterschilderung danach & $\begin{aligned} 0= & \text { unbekannt } \\
1= & \text { nicht zutreffend, da tot } \\
2= & \text { konkrete Schilderung von Wut und } \\
& \text { Rachegedanken } \\
3= & \text { keine Erinnerung } \\
4= & \text { Derealisation, Depersonalisation } \\
5= & \text { sonstige psychopathologische } \\
& \text { Auffälligkeiten }\end{aligned}$ \\
\hline 68 & Verurteilung & $\begin{array}{l}0=\text { unbekannt } \\
1=\text { Geldstrafe, Bewährungsstrafe } \\
2=\text { Strafhaft } \\
3=\text { Maßregelvollzug } \\
\mathrm{x}=\text { tot }\end{array}$ \\
\hline $69-71$ & Strafdauer & (in Monaten, dreistellig, $\mathrm{x}=$ unbekannt) \\
\hline 72 & Exkulpation & $\begin{array}{l}0=\text { unbekannt } \\
1=\text { nicht zutreffend } \\
2=\text { nicht exkulpiert } \\
3=\text { teilexkulpiert, } \S 21 \mathrm{StGB} \\
4=\text { vollständig exkulpiert, } \S 20 \mathrm{StGB}, \mathrm{x}=\text { tot }\end{array}$ \\
\hline
\end{tabular}


Tabelle A 2: Auflistung der ausgewerteten Presseartikel

\begin{tabular}{|c|c|c|c|c|c|c|}
\hline $\begin{array}{l}\text { Ifd. } \\
\text { Nr. }\end{array}$ & Quelle & Artikelüberschrift & Tatort & Was ist passiert? & Ausgang/Schäden & $\begin{array}{l}\text { Echte } \\
\text { Amok- } \\
\text { fahrt? }\end{array}$ \\
\hline 1 & TA 20.02.2001 & Amokfahrt & $\begin{array}{l}\text { B7 Weimar Richtung Erfurt } \\
\text { und Stadtgebiet Erfurt }\end{array}$ & $\begin{array}{l}\text { 30-jähriger, psychisch auffälliger Mann fährt mit } \\
\text { hoher Geschwindigkeit, mehrere Unfälle, zuletzt } \\
\text { Flucht vor der Polizei }\end{array}$ & $\begin{array}{l}\text { Einweisung in die Psychia- } \\
\text { trie, hoher Sachschaden }\end{array}$ & nein \\
\hline 2 & OTZ 21.03.2001 & Amokfahrt & Saalfeld & $\begin{array}{l}\text { 48-jähriger, alkoholisierter und psychisch auffälliger } \\
\text { Mann fährt mit hoher Geschwindigkeit, Flucht vor } \\
\text { der Polizei, gerät auf Gegenfarbahn, Frontalcrash }\end{array}$ & $\begin{array}{l}\text { Unfallfahrer Psychiatrie- } \\
\text { einweisung, } 3 \text { Personen } \\
\text { verletzt, hoher Sachscha- } \\
\text { den }\end{array}$ & nein \\
\hline 3 & TA 27.03.2001 & Amokfahrt & $\begin{array}{l}\text { Kyffhäuserkreis, } \\
\text { Stadt Greußen }\end{array}$ & $\begin{array}{l}\text { junger, drogenintoxikierter Fahrer, mehrere Straf- } \\
\text { tatbestände (kein Nummernschild, Tankbetrug, } \\
\text { Rasen und Überholen trotz Gegenverkehr), Frontal- } \\
\text { crash, Fahrerflucht }\end{array}$ & hoher Sachschaden & nein \\
\hline 4 & TA 26.06.2001 & In Lebensgefahr & Unstrut Hainich Kreis & $\begin{array}{l}\text { 29-jähriger Autofahrer in Rechtskurve auf Gegen- } \\
\text { fahrbahn geraten, Frontalcrash mit entgegenkom- } \\
\text { mendem Pkw }\end{array}$ & $\begin{array}{l}2 \text { Schwerverletzte, hoher } \\
\text { Sachschaden }\end{array}$ & nein \\
\hline $5 / 1$ & TA 19.07.2001 & $\begin{array}{l}\text { Gefährlicher Tun- } \\
\text { nelblick }\end{array}$ & Landkreis Gotha & $\begin{array}{l}\text { 25-jähriger Autofahrer in gestohlenem BMW u. } \\
\text { ohne Führerschein unterwegs, Flucht vor der Poli- } \\
\text { zei, Auffahrunfall in einen Polizeiwagen als Straßen- } \\
\text { sperre }\end{array}$ & $\begin{array}{l}\text { Haftstrafe } 2 \text { Jahre } 2 \text { Mona- } \\
\text { te, hoher Sachschaden }\end{array}$ & nein \\
\hline $5 / 2$ & TA 19.07.2001 & Fahrerflucht & $\begin{array}{l}\text { Landkreis Schmalkalden- } \\
\text { Meiningen }\end{array}$ & $\begin{array}{l}\text { Autodiebstahl, Flucht vor der Polizei, alkoholisierter } \\
\text { und drogenintoxikierter Fahrer }\end{array}$ & $\begin{array}{l}2 \text { verletzte Polizisten, ho- } \\
\text { her Sachschaden }\end{array}$ & nein \\
\hline $5 / 3$ & TA 19.07.2001 & Fahrerflucht & Landkreis Hildburghausen & $\begin{array}{l}\text { 20-jähriger Autofahrer, Flucht vor der Polizei, Auf- } \\
\text { fahrunfall (Frontalcrash gegen Hauswand) }\end{array}$ & $\begin{array}{l}2 \text { Leichtverletzte, hoher } \\
\text { Sachschaden, Täter in Un- } \\
\text { tersuchungshaft }\end{array}$ & nein \\
\hline 6 & TA 23.07.2001 & Geisterfahrt & A4 Höhe Eisenach & $\begin{array}{l}\text { 28-jähriger ortsunkundiger Autofahrer fährt falsch } \\
\text { auf die } A B \text { auf, Kollision mit } 3 \text { anderen Pkw }\end{array}$ & $\begin{array}{l}\text { Tod des Geisterfahrers, } \\
\text { mehrere Schwerverletzte, } \\
\text { hoher Sachschaden }\end{array}$ & nein \\
\hline
\end{tabular}




\begin{tabular}{|c|c|c|c|c|c|c|}
\hline $\begin{array}{l}\text { Ifd. } \\
\text { Nr. }\end{array}$ & Quelle & Artikelüberschrift & Tatort & Was ist passiert? & Ausgang/Schäden & $\begin{array}{l}\text { Echte } \\
\text { Amok- } \\
\text { fahrt? }\end{array}$ \\
\hline 7 & TA 30.07.2001 & Amokfahrt & $\begin{array}{l}\text { Landkreis Nordhausen, } \\
\text { Stadt Ellrich }\end{array}$ & $\begin{array}{l}\text { 20-jähriger, alkoholisierter Autofahrer, nächtliche } \\
\text { Trunkenheitsfahrt }\end{array}$ & hoher Sachschaden & nein \\
\hline 8 & OTZ 04.08.2001 & Todesfahrt & $\begin{array}{l}\text { Oberweißbach, Landkreis } \\
\text { Saalfeld-Rudolstadt }\end{array}$ & $\begin{array}{l}\text { 18-jähriger Autofahrer, hohe Geschwindigkeit, in } \\
\text { Linkkurve abgekommen und } 4 \text { Meter in die Tiefe } \\
\text { gestürzt }\end{array}$ & $\begin{array}{l}\text { Tod des Fahrers, Auto To- } \\
\text { talschaden }\end{array}$ & nein \\
\hline 9 & TLZ 30.11.2001 & Falschfahrer & Landkreis Gotha & $\begin{array}{l}\text { in Kurve auf Gegenfahrbahn gekommen, entgegen- } \\
\text { kommender Fahrer versucht auszuweichen und } \\
\text { prallt dabei gegen Baum, Unfallfahrer begeht Un- } \\
\text { fallflucht }\end{array}$ & $\begin{array}{l}\text { ein Leichtverletzter, hoher } \\
\text { Sachschaden }\end{array}$ & nein \\
\hline 10 & TA 08.12.2001 & Unfallfahrer starb & Landkreis Nordhausen & $\begin{array}{l}\text { 19-jähriger Autofahrer, hohe Geschwindigkeit, in } \\
\text { Rechtskurve auf Gegenfahrbahn geraten, Frontal- } \\
\text { crash }\end{array}$ & $\begin{array}{l}\text { Tod des Unfallfahrers, ho- } \\
\text { her Sachschaden }\end{array}$ & nein \\
\hline 11 & TA 18.12.2001 & Unfallfahrer & Wartburgkreis & $\begin{array}{l}\text { 27-jähriger alkoholisierter Autofahrer in Rechtskur- } \\
\text { ve auf Gegenfahrbahn geraten, Frontalcrash, Unfall- } \\
\text { flucht }\end{array}$ & $\begin{array}{l}\text { ein Leichtverletzter, hoher } \\
\text { Sachschaden }\end{array}$ & nein \\
\hline 12 & TA 28.12.2001 & Amokfahrer & B7 Landkreis Apolda & $\begin{array}{l}\text { 19-Jähriger mit gestohlenem Pkw frontal gegen } \\
\text { Kleintransporter gefahren }\end{array}$ & $\begin{array}{l}\text { ein Toter (Unfallfahrer), } \\
\text { drei Schwerverletzte (Un- } \\
\text { fallbeteiligte im Kleintrans- } \\
\text { porte), hoher Sachschaden }\end{array}$ & nein \\
\hline 13 & TA 15.01.2002 & Amokfahrer & $\begin{array}{l}\text { B7 zwischen Apolda und } \\
\text { Weimar }\end{array}$ & $\begin{array}{l}\text { 19-jähriger Autodieb als Fahrer (und drei Tatbetei- } \\
\text { ligte als Beifahrer) auf der Flucht vor der Polizei }\end{array}$ & $\begin{array}{l}\text { Festnahme der Diebe, } \\
\text { keine Schäden }\end{array}$ & nein \\
\hline 14 & TA 07.02.2002 & Amokfahrt & Artern/Kyffhäuserkreis & $\begin{array}{l}\text { 31-jähriger Fahrer ohne Führerschein auf der } \\
\text { Flucht vor der Polizei (mehrere Haftbefehle gegen } \\
\text { ihn lagen bereits vor) }\end{array}$ & $\begin{array}{l}\text { keine Schäden, Fluchtfah- } \\
\text { rer kam in Haft }\end{array}$ & nein \\
\hline 15 & TA 04.03.2002 & tödlicher Unfall & Weimar & $\begin{array}{l}\text { 22-jähriger Raser in Rechtskurve auf Gegenfahr- } \\
\text { bahn geraten und Frontalcrash verursacht }\end{array}$ & $\begin{array}{l}1 \text { Toter (Beifahrer des Un- } \\
\text { fallfahrers) und } 2 \text { Schwer- } \\
\text { verletzte, hoher Sachscha- } \\
\text { den }\end{array}$ & nein \\
\hline
\end{tabular}




\begin{tabular}{|c|c|c|c|c|c|c|}
\hline $\begin{array}{l}\text { Ifd. } \\
\text { Nr. }\end{array}$ & Quelle & Artikelüberschrift & Tatort & Was ist passiert? & Ausgang/Schäden & $\begin{array}{l}\text { Echte } \\
\text { Amok- } \\
\text { fahrt? }\end{array}$ \\
\hline 16 & TLZ 08.04.2002 & Amokfahrt & A4 bei Eisenach & $\begin{array}{l}\text { 23-jähriger Autodieb (plus Geiselnahme des Fah- } \\
\text { rers), verfolgt von der Polizei, mit hoher Geschwin- } \\
\text { digkeit auf Stauende aufgefahren }\end{array}$ & $\begin{array}{l}\text { Sachschäden, keine Perso- } \\
\text { nenschäden }\end{array}$ & nein \\
\hline 17 & OTZ 17.05.2002 & Geisterfahrer & Autobahn A4 bei Gera & $\begin{array}{l}\text { ortsunkundiger 26-jähriger polnischer Autofahrer } \\
\text { falsch auf die Autobahn aufgefahren }\end{array}$ & keine & nein \\
\hline 18 & TA 16.10 .2002 & Amokfahrt & B4 Nähe Nordhausen & $\begin{array}{l}\text { alkoholisierter Autofahrer auf der Flucht vor der } \\
\text { Polizei }\end{array}$ & keine, AAK vor Ort: $1,78 \%$ o & nein \\
\hline 19 & TA 16.12.2002 & Amokfahrt & Weimar Stadtgebiet & $\begin{array}{l}\text { 39-jähriger, alkoholisierter Autofahrer, der durch } \\
\text { seine Fahrweise diverse Verkehrsleiteinrichtungen } \\
\text { beschädigte }\end{array}$ & $\begin{array}{l}\text { Sachschäden in Höhe von } \\
10.000 \text { Euro, AAK vor Ort: } \\
2,10 \% \text { o }\end{array}$ & nein \\
\hline 20 & OTZ 01.03.2003 & Amokfahrt & $\begin{array}{l}\text { Stadtgebiet Lobenstein und } \\
\text { B281 Saale-Orla-Kreis }\end{array}$ & $\begin{array}{l}\text { Fahren mit überhöhter Geschwindigkeit, Überholen } \\
\text { einer Fahrzeugkolonne und Verursachung eines } \\
\text { Frontalcrashs (plus Fahrerflucht) }\end{array}$ & $\begin{array}{l}\text { Sachschäden in Höhe von } \\
7.000 \text { Euro, Fahndung nach } \\
\text { dem Unfallfahrer }\end{array}$ & nein \\
\hline 21 & OTZ 27.03.2003 & Todesfahrt & Landstraße Landkreis Gera & $\begin{array}{l}\text { 16-Jähriger ohne Führerschein und drei andere } \\
\text { Jugendliche als Beifahrer mit überhöhter Ge- } \\
\text { schwindigkeit in Linkskurve von Fahrbahn abge- } \\
\text { kommen und gegen Baum geprallt }\end{array}$ & $\begin{array}{l}1 \text { Toter, } 2 \text { Schwer-, } 1 \\
\text { Leichtverletzter, Auto To- } \\
\text { talschaden }\end{array}$ & nein \\
\hline 22 & TA 09.05.2003 & Amokfahrer & $\begin{array}{l}\text { Ilmenau/B88 Richtung } \\
\text { Gehren, Ilmkreis }\end{array}$ & $\begin{array}{l}\text { betrunkener Autofahrer auf der Flucht vor der Poli- } \\
\text { zei }\end{array}$ & $\begin{array}{l}\text { AAK vor Ort: } 3,46 \% \text {, } \\
\text { Schäden nicht bekannt }\end{array}$ & nein \\
\hline 23 & TA 10.05.2003 & Todesfahrt & Landstraße Landkreis Weimar & $\begin{array}{l}\text { betrunkener Autofahrer in Rechtskurve Frontalcrash } \\
\text { Gegenfahrbahn }\end{array}$ & $\begin{array}{l}\text { Atemalkoholtest Falschfah- } \\
\text { rer: } 2,35 \% \text { \%, } 1 \text { Toter (Bei- } \\
\text { fahrer), } 1 \text { Schwerverletzter } \\
\text { (Unfallgegner), Unfallfah- } \\
\text { rer: Freiheitsstrafe } 1 \text { Jahr } \\
\text { und } 10 \text { Monate auf Bewäh- } \\
\text { rung, Führerscheinverlust }\end{array}$ & nein \\
\hline 24 & TA 12.06.2003 & 2 Schwerverletzte & $\begin{array}{l}\text { B247 zwischen Dingelstädt } \\
\text { und Mühlhausen }\end{array}$ & $\begin{array}{l}\text { Unfallfahrer aus ungeklärten Gründen auf Gegen- } \\
\text { fahrbahn gefahren, Frontalcrash }\end{array}$ & $\begin{array}{l}2 \text { Schwerverletzte (Unfall- } \\
\text { fahrer, Unfallgegner), Sach- } \\
\text { schäden: } 20.000 \text { Euro }\end{array}$ & nein \\
\hline
\end{tabular}




\begin{tabular}{|c|c|c|c|c|c|c|}
\hline $\begin{array}{l}\text { Ifd. } \\
\text { Nr. }\end{array}$ & Quelle & Artikelüberschrift & Tatort & Was ist passiert? & Ausgang/Schäden & $\begin{array}{l}\text { Echte } \\
\text { Amok- } \\
\text { fahrt ? }\end{array}$ \\
\hline 25 & TA 25.06.2003 & $\begin{array}{l}\text { Aggressiver Auto- } \\
\text { fahrer }\end{array}$ & $\begin{array}{l}\text { Neuhaus, Landkreis Sonne- } \\
\text { berg }\end{array}$ & $\begin{array}{l}\text { 20-jähriger, aggressiver Autofahrer, der nach einem } \\
\text { Streit mit einem Jugendlichen versuchte, diesen mit } \\
\text { seinem Auto umzufahren }\end{array}$ & $\begin{array}{l}\text { Fahrerflucht, durch recht- } \\
\text { zeitiges Ausweichen des } \\
\text { Opfers keine Personen- } \\
\text { schäden, Anzeige wegen } \\
\text { versuchten Totschlags }\end{array}$ & nein \\
\hline 26 & TA 14.07.2003 & Todesfahrt & B2 bei Gera & $\begin{array}{l}\text { 33-jähriger Motoradfahrer in Kurve auf Gegenfahr- } \\
\text { bahn geraten, Frontalcrash mit Pkw }\end{array}$ & $\begin{array}{l}\text { 24-jähriger Unfallgegner } \\
\text { getötet, Unfallverursacher } \\
\text { lebensgefährlich verletzt, } \\
\text { hohe Sachschäden }\end{array}$ & nein \\
\hline 27 & TA 28.08.2003 & Falschfahrer & $\begin{array}{l}\text { Landkreis Gotha, Landstraße } \\
\text { bei Friedrichswerth }\end{array}$ & $\begin{array}{l}\text { 57-jähriger Falschfahrer, Frontalcrash mit } \\
\text { 28-jähriger, hochschwangerer Unfallgegnerin }\end{array}$ & $\begin{array}{l}\text { zwei Schwerverletzte, ho- } \\
\text { her Sachschaden }\end{array}$ & nein \\
\hline 28 & TA 01.09.2003 & Todesfahrt & $\begin{array}{l}\text { B247 im Landkreis Hild- } \\
\text { burghausen }\end{array}$ & $\begin{array}{l}\text { 20-jähriger Fahrer (mit zwei Beifahrern), überhöhte } \\
\text { Geschwindigkeit, regennasse Straße, in Linkskurve } \\
\text { von der Straße abgekommen und gegen Baum ge- } \\
\text { prallt }\end{array}$ & 3 Tote, hoher Sachschaden & nein \\
\hline 29 & TA 28.11.2003 & Geisterfahrer & B4 Nähe Flughafen Erfurt & $\begin{array}{l}\text { 67-jähriger Autofahrer, bei dichtem Nebel falsche } \\
\text { Auffahrt genommen und zum Geisterfahrer gewor- } \\
\text { den, Frontalcrash mit entgegenkommendem Fahr- } \\
\text { zeug }\end{array}$ & $\begin{array}{l}1 \text { Toter (Beifahrerin), } 1 \\
\text { Schwerverletzter (Geister- } \\
\text { fahrer), } 1 \text { Leichtverletzter } \\
\text { (Unfallgegner), hoher Sach- } \\
\text { schaden }\end{array}$ & nein \\
\hline 30 & TLZ 02.12.2003 & Todesfahrt & B7 Landkreis Gotha & $\begin{array}{l}\text { 22-jähriger Autofahrer, überhöhte Geschwindigkeit, } \\
\text { in Rechtskurve aus der Fahrbahn getragen und } \\
\text { gegen Baum geprallt }\end{array}$ & $\begin{array}{l}\text { Unfallfahrer getötet, Unfal- } \\
\text { lauto Totalschaden }\end{array}$ & nein \\
\hline 31 & TLZ 02.12.2003 & Todesfahrt & B84 Wartburgkreis & $\begin{array}{l}\text { Fahrer mit überhöhter Geschwindigkeit in Linkskur- } \\
\text { ve von der Fahrbahn abgekommen und gegen } \\
\text { Baum geprallt }\end{array}$ & $\begin{array}{l}\text { Fahrer tödlich verletzt, } \\
\text { Fahrzeug Totalschaden }\end{array}$ & nein \\
\hline
\end{tabular}




\begin{tabular}{|c|c|c|c|c|c|c|}
\hline $\begin{array}{l}\text { Ifd. } \\
\text { Nr. }\end{array}$ & Quelle & Artikelüberschrift & Tatort & Was ist passiert? & Ausgang/Schäden & $\begin{array}{l}\text { Echte } \\
\text { Amok- } \\
\text { fahrt? }\end{array}$ \\
\hline 32 & TLZ 31.01.2004 & Amokfahrt & $\begin{array}{l}\text { A4 zwischen Stadtroda und } \\
\text { Jena Lobeda }\end{array}$ & $\begin{array}{l}\text { 42-jähriger Autofahrer fährt auf anderes Auto auf, } \\
\text { fährt weiter, dann nochmals mit hoher Geschwin- } \\
\text { digkeit auf anderen Pkw auf, Totalschaden, dann } \\
\text { Frontalcrash mit Lkw }\end{array}$ & $\begin{array}{l}\text { Tod des Unfallfahrers, ein } \\
\text { Leichtverletzter, hoher } \\
\text { Sachschaden }\end{array}$ & fraglich \\
\hline 33 & TLZ 13.10.2004 & Amokfahrt & Landstraße Erfurt Land & $\begin{array}{l}\text { 55-jähriger Autofahrer missachtet Baustellenab- } \\
\text { sperrung und begeht Fahrerflucht vor der Polizei }\end{array}$ & $\begin{array}{l}\text { Sachschäden (Zerstörung } \\
\text { der Baustellenabsperrung) }\end{array}$ & nein \\
\hline 34 & TA 15.10 .2004 & Geisterfahrer & $\begin{array}{l}\text { A4 Nähe Landesgrenze zu } \\
\text { Hessen }\end{array}$ & $\begin{array}{l}\text { 48-jähriger Autofahrer auf der Autobahn plötzlich } \\
\text { angehalten, gewendet und als Geisterfahrer weiter- } \\
\text { gefahren, nach } 5 \text { Kilometern frontal auf ein entge- } \\
\text { genkommendes Auto aufgefahren }\end{array}$ & $\begin{array}{l}\text { Unfallfahrer tot, Unfallgeg- } \\
\text { nerin schwerverletzt, ho- } \\
\text { her Sachschaden }\end{array}$ & möglich \\
\hline 35 & TLZ 08.11.2004 & Amokfahrt & $\begin{array}{l}\text { Landstraße Eisenach Neukir- } \\
\text { chen }\end{array}$ & $\begin{array}{l}\text { 26-jährige Autofahrerin fuhr } 2 \text { Fußgänger an, } \\
\text { beging Fahrerflucht, rammte danach } 2 \text { geparkte } \\
\text { Autos, geriet dann auf Gegenfahrbahn und stieß } \\
\text { mit entgegenkommendem Fahrzeug zusammen }\end{array}$ & $\begin{array}{l}\text { diverse Sachschäden, zwei } \\
\text { Leichtverletzte, Atemalko- } \\
\text { holtest Unfallfahrerin: } 1,8 \\
\%\end{array}$ & nein \\
\hline 36 & TA 01.03.2005 & $\begin{array}{l}\text { 2,68 Promille } \\
\text { Restalkohol }\end{array}$ & Stadtgebiet Sondershausen & $\begin{array}{l}\text { 39-jähriger Pkw-Fahrer geriet auf die Gegenfahr- } \\
\text { bahn und prallte gegen einen Lkw, danach Fahrer- } \\
\text { flucht des Unfallfahrers }\end{array}$ & $\begin{array}{l}\text { Sachschäden, AAK: vor Ort: } \\
2,68 \% \text { o }\end{array}$ & nein \\
\hline 37 & TA 05.04.2005 & $\begin{array}{l}\text { Im geschlossenen } \\
\text { Vollzug }\end{array}$ & B19 Wartburgkreis & Amokfahrt über 20 Kilometer & $\begin{array}{l}1 \text { Toter, Sachschäden über } \\
100.000 \text { Euro }\end{array}$ & ja \\
\hline 38 & TA 07.04.2005 & Geschockt & Fortsetzung des TA-Artikels & vom 05.04.2005 über denselben Amokfahrer & & ja \\
\hline 39 & OTZ 19.04.2005 & Geisterfahrer & B92 Stadtring Gera & $\begin{array}{l}\text { 40-jähriger ortsunkundiger Fahrer falsch aufgefah- } \\
\text { ren, danach als Geisterfahrer, Frontalzusammen- } \\
\text { stoß mit Pkw }\end{array}$ & $\begin{array}{l}1 \text { Schwerverletzter (Beifah- } \\
\text { rerin), } 2 \text { Leichtverletzte, } \\
\text { hoher Sachschaden }\end{array}$ & nein \\
\hline 40 & TLZ 30.05.2005 & Todesfahrt & A4 Höhe Gera & 79-jähriger Autofahrer auf Lkw aufgefahren & $\begin{array}{l}\text { Fahrer und Beifahrerin tot, } \\
\text { Fahrzeug Totalschaden }\end{array}$ & nein \\
\hline
\end{tabular}




\begin{tabular}{|c|c|c|c|c|c|c|}
\hline $\begin{array}{l}\text { Ifd. } \\
\text { Nr. }\end{array}$ & Quelle & Artikelüberschrift & Tatort & Was ist passiert? & Ausgang/Schäden & $\begin{array}{l}\text { Echte } \\
\text { Amok- } \\
\text { fahrt? }\end{array}$ \\
\hline 41 & TLZ 29.10.2005 & Geisterfahrer & B80 Stadtgebiet Heiligenstadt & $\begin{array}{l}\text { Mercedes fährt nachts ohne Licht auf der Gegen- } \\
\text { fahrbahn, fährt gezielt auf entgegenkommendes } \\
\text { Fahrzeug zu, Unfallgegner weicht auf Gehweg aus, } \\
\text { Fahrerflucht }\end{array}$ & $\begin{array}{l}\text { Sachschaden beim Unfall- } \\
\text { gegner }\end{array}$ & möglich \\
\hline 42 & TA 30.03.2006 & Geisterfahrer & A71 Nähe Erfurt & $\begin{array}{l}\text { 76-jähriger orientierungsloser Autofahrer (bekann- } \\
\text { te Demenz) auf Gegenfahrbahn unterwegs, Frontal- } \\
\text { crash }\end{array}$ & $\begin{array}{l}\text { Sachschaden } 17.000 \text { Euro, } \\
\text { keine Verletzten }\end{array}$ & nein \\
\hline 43 & TA 04.05.2006 & Amokfahrt & Stadtgebiet IImenau & $\begin{array}{l}\text { 25-Jähriger mit unterschlagenem Auto von Auto- } \\
\text { vermietung auf der Flucht vor der Polizei, mehrere } \\
\text { Fahrzeuge gerammt bei hoher Geschwindigkeit, } \\
\text { zuletzt im Gegenverkehr geraten }\end{array}$ & $\begin{array}{l}\text { keine Verletzten, Sach- } \\
\text { schäden in Höhe von } \\
25.000 \text { Euro }\end{array}$ & nein \\
\hline 44 & TA 09.06.2006 & Geisterfahrerin & A71 Erfurt-Stotternheim & $\begin{array}{l}\text { 70-jährige ortsfremde Autofahrerin falsch auf die } \\
\text { Autobahn aufgefahren }\end{array}$ & $\begin{array}{l}\text { keine (von der Polizei ge- } \\
\text { stoppt, wirkte verwirrt) }\end{array}$ & nein \\
\hline 45 & OTZ 30.05.2006 & Geisterfahrerunfall & A93 bei Hirschberg & $\begin{array}{l}\text { 39-jähriger Fahrer auf der Gegenfahrbahn unter- } \\
\text { wegs, 1.Unfall, Fahrerflucht, Wenden in korrekte } \\
\text { Fahrtrichtung, danach weiterer Unfall, nochmaliges } \\
\text { Wenden gegen die Fahrtrichtung, Beinahe Unfall, } \\
\text { nochmalige Fahrerflucht }\end{array}$ & $\begin{array}{l}\text { von der Polizei gestoppt, } \\
\text { keinerlei Papiere dabei, } \\
\text { Sachschäden }\end{array}$ & nein \\
\hline 46 & TA 30.05.2006 & $\begin{array}{l}\text { Im geschlossenen } \\
\text { Vollzug }\end{array}$ & Forts. der Berichterstattung & über Amokfahrt (s. Ifd. Nrn. 37 + 38) & & ja \\
\hline 47 & TA 01.06.2006 & $\begin{array}{l}\text { Im geschlossenen } \\
\text { Vollzug }\end{array}$ & Forts. der Berichterstattung & über Amokfahrt (s. Ifd. Nrn. 37, 38, 46) & & ja \\
\hline 48 & OTZ 16.08.2006 & Amokfahrt & Stadtgebiet Lobenstein & 50-jährige Frau rammt abgestellten Pkw & $\begin{array}{l}\text { schwerstalkoholisierte } \\
\text { Fahrerin, (AAK nicht durch- } \\
\text { führbar), Sachschaden } \\
4.000 €\end{array}$ & nein \\
\hline 49 & TA 13.10.2006 & Irrfahrt im Nebel & A71 Nähe Erfurter Kreuz & $\begin{array}{l}\text { 52-jähriger Autofahrer im dichten Nebel falsch auf } \\
\text { die Autobahn aufgefahren, Frontalcrash mit Klein- } \\
\text { transporter }\end{array}$ & $\begin{array}{l}2 \text { Schwerverletzte, hoher } \\
\text { Sachschaden }\end{array}$ & nein \\
\hline
\end{tabular}




\begin{tabular}{|c|c|c|c|c|c|c|}
\hline $\begin{array}{l}\text { Ifd. } \\
\text { Nr. }\end{array}$ & Quelle & Artikelüberschrift & Tatort & Was ist passiert? & Ausgang/Schäden & $\begin{array}{l}\text { Echte } \\
\text { Amok- } \\
\text { fahrt ? }\end{array}$ \\
\hline 50 & TLZ 21.11.2006 & Amokfahrt & A4 Höhe Apolda & $\begin{array}{l}\text { 41-jähriger, alkoholisierter Autofahrer verfolgt von } \\
\text { der Polizei }\end{array}$ & Atemalkoholtest 1,26 \%。 & nein \\
\hline 51 & TA 02.01.2007 & $\begin{array}{l}\text { Betrunkener rast in } \\
\text { Fußgängergruppe }\end{array}$ & Stadtgebiet Sondershausen & $\begin{array}{l}\text { 42-jähriger Autofahrer rast in Fußgängergruppe } \\
\text { und begeht danach Unfallflucht }\end{array}$ & $\begin{array}{l}1 \text { Person (Fußgängerin) } \\
\text { tödlich verletzt, Unfallfah- } \\
\text { rer AAK 1,44 \%o, Strafver- } \\
\text { fahren }\end{array}$ & nein \\
\hline 52 & OTZ 19.07.2007 & Geisterfahrer & B281 bei Saalfeld & $\begin{array}{l}\text { 87-jähriger Autofahrer bei neuer Ortsumfahrung } \\
\text { falsch aufgefahren und danach als Geisterfahrer } \\
\text { unterwegs gewesen }\end{array}$ & $\begin{array}{l}\text { keine (rechtzeitig durch die } \\
\text { Polizei gestoppt worden) }\end{array}$ & nein \\
\hline 53 & OTZ 30.11.2007 & Geisterfahrer & A9 Landesgrenze Bayern & $\begin{array}{l}\text { Richtungsirrtum von } 2 \text { polnischen Autofahrern, } \\
\text { wendeten daraufhin auf dem Standstreifen und } \\
\text { waren danach als Falschfahrer unterwegs }\end{array}$ & $\begin{array}{l}\text { Keine Schäden, rechtzeitig } \\
\text { durch die Polizei gestoppt }\end{array}$ & nein \\
\hline 54 & OTZ 23.05.2009 & Geisterfahrer & B7 Jena Richtung Stadtroda & $\begin{array}{l}\text { 23-jähriger Autofahrer, paranoide Schizophrenie, } \\
\text { will sich mit dem Auto umbringen, fährt abge- } \\
\text { schnallt mit hoher Geschwindigkeit als Geisterfah- } \\
\text { rer auf entgegenkommendes Fahrzeug zu }\end{array}$ & $\begin{array}{l}5 \text { Leichtverletzte (Fahrerin } \\
\text { und ihre } 4 \text { Kinder im ent- } \\
\text { gegen-kommenden Fahr- } \\
\text { zeug), keine schwereren } \\
\text { Schäden durch Ausweich- } \\
\text { manöver der Unfallgegne- } \\
\text { rin }\end{array}$ & ja \\
\hline 55 & TA 09.06.2008 & $\begin{array}{l}\text { Betrunkener } \\
\text { Geisterfahrer }\end{array}$ & Stadtgebiet Erfurt & betrunkener Falschfahrer von der Polizei gestoppt & $\begin{array}{l}\text { keine, Atemalkoholtest } \\
1,09 \%\end{array}$ & nein \\
\hline 56 & TA 05.07.2008 & Eine wilde Jagd & Stadtgebiet IImenau & $\begin{array}{l}\text { junger Autofahrer fährt mit hoher Geschwindigkeit } \\
\text { durch Innenstadt, verursacht mehrere Unfälle, Ver- } \\
\text { folgung durch die Polizei, wurde am Ende gestellt, } \\
\text { Drogentest: Ecstasy }\end{array}$ & hoher Sachschaden & nein \\
\hline 57 & OTZ 22.09.2008 & Geisterfahrer & $\begin{array}{l}\text { A9 Richtung Berlin Höhe } \\
\text { Hermsdorfer Kreuz }\end{array}$ & $\begin{array}{l}\text { 54-jähriger Autofahrer fährt als Geisterfahrer auf } \\
\text { der Autobahn, dabei mehrere Unfälle verursacht } \\
\text { durch Ausweichen anderer Fahrzeuge (insgesamt } \\
\text { 19) }\end{array}$ & $\begin{array}{l}\text { von der Polizei gestellt, 1,9 } \\
\% \text { AAT, } 4 \text { Leicht-verletzte, } \\
\text { Sachschaden ca. } 120.000 \\
\text { Euro }\end{array}$ & nein \\
\hline
\end{tabular}




\begin{tabular}{|c|c|c|c|c|c|c|}
\hline $\begin{array}{l}\text { Ifd. } \\
\text { Nr. }\end{array}$ & Quelle & Artikelüberschrift & Tatort & Was ist passiert? & Ausgang/Schäden & $\begin{array}{l}\text { Echte } \\
\text { Amok- } \\
\text { fahrt? }\end{array}$ \\
\hline 58 & TA 20.10.2008 & $\begin{array}{l}\text { Tragisches Ende } \\
\text { eines Spaziergangs }\end{array}$ & Dorfstraße Landkreis Apolda & $\begin{array}{l}\text { Autofahrer erlitt epileptischen Anfall, verlor die } \\
\text { Kontrolle über sein Fahrzeug und raste auf den } \\
\text { Gehsteig }\end{array}$ & 2 Tote (Fußgänger) & nein \\
\hline 59 & OTZ 09.02.2009 & Geisterfahrer & $\begin{array}{l}\text { A4 Jena Richtung Hermsdor- } \\
\text { fer Kreuz }\end{array}$ & $\begin{array}{l}\text { polnischer Kleintransporter als Geisterfahrer un- } \\
\text { terwegs, wurde durch die Polizei gestoppt, sämtli- } \\
\text { che Insassen stark alkoholisiert (auch der Fahrer) }\end{array}$ & $\begin{array}{l}\text { Sachschäden, keine Perso- } \\
\text { nenschäden }\end{array}$ & nein \\
\hline 60 & TA 03.03.2009 & Amokfahrt & Landstraße Landkreis Apolda & $\begin{array}{l}\text { Unbekannte entwendeten Bagger von Baufirma } \\
\text { und demolierten damit einen Radweg }\end{array}$ & Sachschäden & nein \\
\hline 61 & TA 06.03.2009 & Unfallfahrer & Stadtgebiet Erfurt & $\begin{array}{l}\text { junger Autofahrer, Zusammenstoß mit einem ande- } \\
\text { ren Auto, daraufhin Fahrerflucht, durch die Polizei } \\
\text { gefasst, stand unter Drogen }\end{array}$ & $\begin{array}{l}\text { Sachschaden ca. } 7.000 \\
\text { Euro, keine Personenschä- } \\
\text { den }\end{array}$ & nein \\
\hline 62 & TA 16.03.2009 & Die Helden der A9 & $\begin{array}{l}\text { A9 kurz vor dem } \\
\text { Hermsdorfer Kreuz }\end{array}$ & $\begin{array}{l}\text { 80-jähriger verwirrter orientierungsloser Autofah- } \\
\text { rer als Geisterfahrer unterwegs, wurde durch } 2 \text { Lkw- } \\
\text { und einen Reisebusfahrer an der Weiterfahrt gehin- } \\
\text { dert }\end{array}$ & keine & nein \\
\hline 63 & TA 17.09.2009 & Verfolgungsjagd & $\begin{array}{l}\text { Landkreis Heiligenstadt, } \\
\text { Ortskern Niederorschel }\end{array}$ & $\begin{array}{l}\text { Im Presseartikel als Amokfahrt bezeichnete Flucht } \\
\text { eines 21-jährigen Autofahrern vor der Polizei }\end{array}$ & $\begin{array}{l}\text { keine Verletzten, hoher } \\
\text { Sachschaden }\end{array}$ & nein \\
\hline 64 & TA 24.08.2009 & Tragische Bilanz & $\begin{array}{l}\text { Ortsausgang Sömmerda } \\
\text { Richtung A71 }\end{array}$ & $\begin{array}{l}\text { 19-Jähriger unter Drogeneinfluss mit Kleinbus un- } \\
\text { gebremst auf } 2 \text { verkehrs-bedingt haltende Fahrzeu- } \\
\text { ge aufgefahren }\end{array}$ & $\begin{array}{l}5 \text { z. T. Schwerverletzte, } \\
\text { hoher Sachschaden }\end{array}$ & nein \\
\hline 65 & OTZ 28.09.2009 & Geisterfahrer & $\begin{array}{l}\text { Autobahn A4, Anschlussstelle } \\
\text { Gera-Leumnitz }\end{array}$ & $\begin{array}{l}\text { 84-jähriger, verwirrter Autofahrer falsch aufgefah- } \\
\text { ren, wurde von der Polizei gestellt }\end{array}$ & keine Schäden & nein \\
\hline 66 & TA 29.09.2009 & Horrorunfall & A4 Höhe Stadtroda & Lkw raste ungebremst in Stauende & $\begin{array}{l}1 \text { Toter, } 5 \text { Schwer-verletzte, } \\
\text { sehr hoher Sachschaden }\end{array}$ & $\begin{array}{l}\text { wenig } \\
\text { wahr- } \\
\text { scheinlich }\end{array}$ \\
\hline
\end{tabular}




\begin{tabular}{|c|c|c|c|c|c|c|}
\hline $\begin{array}{l}\text { Ifd. } \\
\text { Nr. }\end{array}$ & Quelle & Artikelüberschrift & Tatort & Was ist passiert? & Ausgang/Schäden & $\begin{array}{l}\text { Echte } \\
\text { Amok- } \\
\text { fahrt? }\end{array}$ \\
\hline 67 & TA 04.11.2009 & $\begin{array}{l}\text { Folgenschwerer } \\
\text { Unfall }\end{array}$ & $\begin{array}{l}\text { Werratal-Kaserne } \\
\text { Bad Salzungen }\end{array}$ & $\begin{array}{l}\text { 26-jähriger Unteroffizier mit Pkw in Soldatengrup- } \\
\text { pe gerast }\end{array}$ & $\begin{array}{l}6 \text { Leicht-, } 7 \text { Schwer- } \\
\text { verletze, Ergebnis der kri- } \\
\text { minalpolizeilichen Ermitt- } \\
\text { lungen: fahrlässiges Fehl- } \\
\text { verhalten des Fahrers }\end{array}$ & nein \\
\hline 68 & TLZ 31.12.2009 & Amokfahrt & Stadtgebiet Weimar & $\begin{array}{l}\text { betrunkener 27-jähriger Autofahrer beschädigt } \\
\text { geparkte Autos und Verkehrsleiteinrichtungen }\end{array}$ & $\begin{array}{l}\text { von der Polizei gestellt, } \\
\text { Atemalkoholtest: } 2,0 \% \text {, } \\
\text { keine Verletzten, hoher } \\
\text { Sachschaden }\end{array}$ & nein \\
\hline 69 & TLZ 26.04.2010 & Amokfahrt & $\begin{array}{l}\text { Waltershausen Landkreis } \\
\text { Gotha }\end{array}$ & $\begin{array}{l}\text { 23-jähriger alkohol- und drogenintoxikierter Auto- } \\
\text { fahrer rammt mehrere geparkte Pkw und Verkehrs- } \\
\text { leiteinrichtungen, danach Fahrerflucht }\end{array}$ & $\begin{array}{l}\text { durch die Polizei gestellt, } \\
\text { AAT: } 2 \% \text { o, Drogentest posi- } \\
\text { tiv }\end{array}$ & nein \\
\hline $70 / 1$ & TA 14.06.2010 & Zwei Geisterfahrten & A71 Höhe Kreuz Erfurt-Nord & $\begin{array}{l}\text { 75-jähriger ortsfremder Autofahrer falsch auf } A B \\
\text { aufgefahren und zum Geister-fahrer geworden, } \\
\text { Frontalcrash mit anderem Pkw }\end{array}$ & $\begin{array}{l}\text { Unfallverursacher tot, } \\
\text { Unfallgegner und Beifahrer } \\
\text { schwer verletzt, hoher } \\
\text { Sachschaden }\end{array}$ & nein \\
\hline $70 / 2$ & TA 14.06.2010 & Zwei Geisterfahrten & A71 Höhe Meiningen-Nord & $\begin{array}{l}\text { 62-jähriger Autofahrer falsch auf die AB aufgefah- } \\
\text { ren, Frontalcrash mit entgegenkommendem Pkw }\end{array}$ & $\begin{array}{l}3 \text { Schwerverletzte (Unfall- } \\
\text { verursacher, Unfallgegner, } \\
\text { Beifahrerin), hoher Sach- } \\
\text { schaden }\end{array}$ & nein \\
\hline 71 & TA 06.08.2010 & Geisterfahrer & $\begin{array}{l}\text { A38 Landesgrenze Sachsen- } \\
\text { Anhalt }\end{array}$ & $\begin{array}{l}\text { 80-jähriger Autofahrer auf der Ausfahrt gewendet } \\
\text { und als Falschfahrer auf die AB zurückgefahren }\end{array}$ & $\begin{array}{l}\text { keine Schäden, durch die } \\
\text { Polizei gestoppt }\end{array}$ & nein \\
\hline 72 & TA 28.08.2010 & Geisterfahrer & B7 Stadtgebiet Erfurt & $\begin{array}{l}\text { 76-jähriger Autofahrer auf der B7 als Falschfahrer } \\
\text { unterwegs, Vermeidung Frontalcrash durch Unfall- } \\
\text { gegner, aber durch Vollbremsung mehrere Auffahr- } \\
\text { unfälle }\end{array}$ & $\begin{array}{l}1 \text { Leichtverletzter, hoher } \\
\text { Sachschaden }\end{array}$ & nein \\
\hline 73 & TA 20.09.2010 & Geisterfahrer & $\begin{array}{l}\text { Stadtgebiet Erfurt, An- } \\
\text { schlussstelle A71 }\end{array}$ & $\begin{array}{l}\text { 55-jähriger Autofahrer musste plötzlich einem } \\
\text { Falschfahrer (silberner Pkw) ausweichen }\end{array}$ & $\begin{array}{l}\text { geringe Sachschäden, keine } \\
\text { Personen-schäden, Fahn- } \\
\text { dung durch die Polizei }\end{array}$ & nein \\
\hline
\end{tabular}




\begin{tabular}{|c|c|c|c|c|c|c|}
\hline $\begin{array}{l}\text { Ifd. } \\
\text { Nr. }\end{array}$ & Quelle & Artikelüberschrift & Tatort & Was ist passiert? & Ausgang/Schäden & $\begin{array}{l}\text { Echte } \\
\text { Amok- } \\
\text { fahrt? }\end{array}$ \\
\hline 74 & OTZ 13.11.2010 & Geisterfahrer & A71 Landesgrenze Bayern & $\begin{array}{l}\text { 30-jähriger Autofahrer mit hoher Geschwindigkeit } \\
\text { als Falschfahrer unterwegs, in Suhl von der Polizei } \\
\text { gestellt, Fahrer stand unter Drogeneinfluss }\end{array}$ & $\begin{array}{l}\text { keine (berichtet wurde } \\
\text { über viele gefährliche Situ- } \\
\text { ationen und Beinahe- } \\
\text { Unfälle unterwegs) }\end{array}$ & nein \\
\hline 75 & TA 27.11.2010 & Geisterfahrer & $\begin{array}{l}\text { A38 Landesgrenze Sachsen- } \\
\text { Anhalt }\end{array}$ & $\begin{array}{l}\text { Falschfahrer mit hoher Geschwindigkeit frontal in } \\
\text { entgegenkommenden Lkw gerast }\end{array}$ & $\begin{array}{l}\text { Unfallverursacher tot, } \\
\text { Unfallgegner unverletzt, } \\
\text { hoher Sachschaden }\end{array}$ & nein \\
\hline 76 & TA 29.11.2010 & Geisterfahrt & Forts .d. Berichterstattung & von Ifd. Nr. 75 & & \\
\hline
\end{tabular}




\section{Literaturverzeichnis}

Åberg L, Rimmö P (1998): Dimensions of abarrant driver behaviour. Ergonomics $\underline{41}, 39$ - 56

Adelt PJ, Grimmer W, Stephan ER: Autofahrer-Typen auf Deutschlands Straßen. Verlag für neue Wissenschaft, Bremerhaven 1999

Adler L: Amok. Eine Studie. belleville Verlag, München 2000

Adler L (2001): Amok im Spektrum homizidal-suizidaler Handlungen. Suizidprophylaxe 28, $103-107$

Adler L (2006): Das Böse und der Fanatismus - Beispiel Amok. NeuroTransmitter Sonderheft $\underline{2,51-55}$

Adler L: Amok in: Suizidalität: Verstehen - Vorbeugen - Behandeln; Wolfersdorf M, Bronisch T, Wedler H; F. Roderer Verlag, Regensburg 2008, 51 - 62

Adler L (2010a): Amok im Spektrum homizidal-suizidaler Handlungen. Suizidprophylaxe $\underline{37}$, $8-14$

Adler L: Amok und School Shooting in: Psychotherapie der Aggression - Keine Angst vor Wut; Bronisch T, Sulz, SKD; CIP-Medien Verlag, München 2010, 64 - 85

Adler L: Neurogenese des Amok in: Neurobiologie forensisch relevanter Störungen; Müller J: W. Kohlhammer Verlag, Stuttgart 2010, 222 - 230

Adler L: Amok. Geschichte und Ergebnisse aus psychiatrischer Perspektive in: Amok und Schulmassaker. Kultur- und medienwissenschaftliche Annäherungen; Junkerjürgen R, Treskow I v; transcipt Verlag, Bielefeld 2015, 17 - 47

Adler L, Lehmann K, Räder K, Schünemann KF (1993): Amokläufer - kontentanalytische Untersuchung an 196 Pressemitteilungen aus industrialisierten Ländern. Fortschr Neurol Psychiat $\underline{61}, 424-433$

Adler L, Lehmann K, Räder K, Schünemann KF, Hajak G (1994): Gibt es Prädiktoren für impulsive, homizidal-suizidale Gewalttaten? Gesundheitswesen $\underline{56}, 548$ - 552 
Adler L, Marx D, Apel H, Wolfersdorf M, Hajak G (2006): Zur Stabilität des „AmokläuferSyndroms“. Fortschr Neurol Psychiatr $\underline{74}, 582$ - 590

Al Bawab A Q, AlQahtani F, McElnay J (2018): Health Care Apps Reported in Newspapers. Content Analysis. JMIR Mhealth Uhealth $\underline{10}, 10218$ - 10237

Asberg M, Traskman L, Thorén P (1976): 5-HIAA in the cerebrospinal fluid. A biochemical suicide predictor. Arch Gen Psychiatr 33, 1193 - 1197

Berlitz J: Daten \& Fakten zum Thema Falschfahrer. ADAC-Falschfahrerstudie. ADAC Verlag, München 2012

Bierwas V, Brühning E, Reichwein HP, Schmid M, Steinhoff H, Weißbrodt G: Untersuchungen zur Verhinderung von Falschfahrten auf Autobahnen, Schlussbericht. Bundesanstalt für Straßenwesen, Köln 1981, 9 - 90

Böker W, Häfner H: Gewalttaten Geistesgestörter. Springer Verlag Berlin Heidelberg New York 1973, $234-273$

Calloway C, Jorgensen CM, Saraiya M, Tsui J (2006): A content analysis of news coverage of the HPV vaccine by U.S. newspapers January 2002 - June 2005. J Womens Health $\underline{17}, 803$ 809

Ellinghaus D, Steinbrecher J: Die Autobahn, Verkehrsweg oder Kampfstätte? Eine Untersuchung über Konflikte und Unfallgeschehen auf Autobahnen im Auftrag der UniroyalEnglebert Reifen GmbH (Uniroyal-Verkehrsuntersuchung Nr. 19), Köln/Aachen 1994, 43 - 45

Färber Be, Färber Br: Methoden und erste Ergebnisse zum Problem Falschfahrten auf Autobahnen in: Berichte aus dem Psychologischen Institut der Universität Tübingen; Psychologisches Institut der Universität Tübingen, Bericht Nr. 2 1980, 3 - 41

Fong G, Frost D, Stansfeld S (2001): Road rage: A psychiatric phenomenon? Soc Psychiatry Psychiatr Epidemiol 36, 277 - 286

Ford R, Moseley AL (1963): Motor vehicle suicides. J Criminol Pol Sc 54, 357 - 359

Gordon H (2004): Psychiatry, the law and death on the roads. Adv Psychiatr Treat, $\underline{10}, 439$ 445 
Greiner A: Verkehrskriminalität in: Lehr- und Studienbriefe Kriminalistik Nr. 17; Greiner A, Polizeiliteratur Verlag Hilden 1995, 3 - 91

Grey EM, Triggs TJ, Haworth NL: Driver aggression: The role of personality, social characteristics, risk and motivation. Report No. CR 81. A study from the Accident Research Centre of the Monash University Monash University Press 1989, 9 - 12

Hamburger E (1969): Vehicular suicidal ideation. Mil Med 134, 441 - 445

Harbort S (1995): Verkehrsunfall als getarnter Selbstmord. Kriminalistik $\underline{3}, 201$ - 204

Imajo T (1983): Suicide by Motor Vehicle. J For Sc $\underline{28}, 83$ - 89

Isherwood J, Adams KS, Hornblow AR (1982): Life event stress, psychosocial factors, suicide attempt and auto-accident proclivity. J Psychosom Res 26, 371 - 383

Kastrup M, Dupont A, Billie M, Lund H (1978): Traffic accidents involving psychiatric patients. Acta Psychiatr Scand $\underline{58}, 30$ - 39

Keskinen E, Pasanen A (1990): Self-destruction in motor vehicle accidents: the proportion of suicides and negligent drivers in fatal motor vehicle accidents in $1974-75$ and $1984-85$ in Finland. J Traffic Med 18, 179 - 185

Kuroda N, Pounder DJ (1994): Suicide on the Roads. J Traffic Med 23, 67 - 70

Laux G, Brunnauer A: Verkehrsdelinquenz aus psychiatrischer und psychologischer Sicht in: Kriminologie und forensische Psychiatrie, Handbuch der forensischen Psychiatrie Bd. 4; Kröber H, Dölling D, Leyhgraf N, Sass H; Steinkopff Verlag, München 2010, 598 - 611

Maag C, Krüger H: Aggressionen im Straßenverkehr in: Berichte der Bundesanstalt für Straßenwesen, Heft M 151; Bundesanstalt für Straßenwesen, Verlag für neue Wissenschaft, Bremerhaven 2003, 8 - 20

MacDonald JM (1964): Suicide and Homicide by Automobile. Am J Psychiatr, 121, 366 - 370

Macmillan J: Deviant Drivers. Saxon House, Westmead 1975, 193 - 201

Mayer RE (1987): Psychological, social and cognitive characteristics of high-risk drivers. Accid Anal Prev $\underline{9}, 1-8$ 
Mayring P: Qualitative Inhaltsanalyse. Grundlagen und Techniken. Beltz Verlag Weinheim und Basel 2010, 26 - 117

Menninger K: Man against himself. Harcourt, Brace \& World, New York 1938, 24 - 83

Michalowski RJ (1975): Violence in the Road: The Crime of Vehicular Homicide. J Res Crime Delinq, $\underline{1,} 31-43$

Nabi H, Consoli SM, Chiron M, Lafont S, Chastang J, Zins M, Lagarde E (2006): Aggressive/hostile personality traits and injury accidents: An eigth-year prospective study of a large cohort of French employees - the GAZEL cohort. Psychol Med 36, 365 - 373

Ohberg A, Penttila A, Lonnqvist J (1997): Driver suicides 1997. Brit J Psychiatr 171, 468 472

Petersen F: Suizid mit dem PKW in Gestalt eines vermeintlichen Verkehrsunfalles. Dissertation Christian-Albrechts-Universität 1974

Phillips DP (1977): Motor vehicle fatalities increase - just after publicized suicide stories. Science $\underline{196}, 1464-1465$

Pokorny AD, Smith JP, Finch JR (1972): Vehicular suicides. Life-threatening behavior 2, $\underline{2}$ $105-119$

Scaramuzza G, Cavegn M: Geisterfahrer. Unfallgeschehen - Interventionen. BfU-Pilotstudie R 0605; Schweizerische Beratungsstelle für Unfallverhütung Bern 2006, 27 - 38

Schmidt CW, Perlin S, Townes W, Fisher RS, Shaffer JW (1972): Characteristics of drivers involved in single-car accidents. A comparative study. Arch Gen Psychiatr $\underline{6}, 800$ - 803

Schmidt CW, Shaffer JW, Zlotowitz HI, Fisher RS (1977): Suicide by vehicular crash. Am J Psychiatr $\underline{134}, 175-178$

Schünemann K: Der sogenannte Amoklauf in der westlichen Welt. Eine statistische Untersuchung von 196 Fällen. Dissertation, Georg-August-Universität Göttingen 1991

Schützhofer B, Litzenberger M, Invanschitz D, Torner F (2008): Zur Persönlichkeit von Geisterfahrern. Psych Öster, $\underline{3 / 4}, 276-281$ 
Shinar D: Psychology on the road. The human factor in traffic. Wiley \& Sons, New York $1978,29-54$

Statistisches Bundesamt: Statistische Jahrbücher für die Bundesrepublik Deutschland. 2002 2011. Statistisches Bundesamt Wiesbaden 2002 - 2011

Tabachnik N, Gussen J, Litmann RE, Peck ML, Tiber N, Wold C: Accident or suicide? Destruction by Automobile. Charles C. Thomas Publisher Springfield, Illinois USA 1973, 5 - 51

Thüringer Landesamt für Statistik: Statistische Jahrbücher Thüringen 2002 - 2011. Thüringer Landesamt für Statistik, Erfurt 2002 - 2011

Whitlock FA: Death on the road: A study in social violence. Tavistock Publications London $1971,9-50$

Wolfgang ME: Patterns in criminal homicide. University of Pennsylvania Press, Philadelphia $1958,79-95$ 


\section{Danksagung}

Mein besonderer Dank gilt meinem Betreuer Herrn Prof. Dr. med. habil. Lothar Adler, der mir aus seinem Forschungsgebiet Amok das Thema „Amokfahrten“ vorschlug. Er begleitete den Entstehungsprozess dieser Arbeit auch noch über seine Emeritierung hinaus mit wichtigen Ratschlägen und Hinweisen. Des Weiteren möchte ich Herrn Prof. Dr. mult. Thomas Meyer für sein außerordentliches Engagement und seine wertvollen Hinweise danken, durch die meine Arbeit eine nochmalige deutliche Verbesserung erfuhr. Und nicht versäumen möchte ich, Frau Elisabeth Goethe, wissenschaftliche Bibliothekarin am Ökumenischen Hainich Klinikum Mühlhausen zu danken, die mich bei der Literaturrecherche zu dieser Arbeit fachkundig beriet und unterstützte. 
